Universidad Nacional de La Plata

Facultad de Ciencias Exactas

Departamento de Matemática

\title{
EL OPERADOR CLIQUE Y LOS GRAFOS PLANARES
}

\author{
Autor: \\ Liliana G. Alcón
}

Director:

Dra. Marisa Gutierrez

Tesis para obtener el título de

DOCTOR EN CIENCIAS EXACTAS

Marzo de 2003 
A mis hijos

Pepe, Lucía y Pupi, mi esposo

Bernardo y mis padres Quique y Lida. 
Quiero expresar mi agradecimiento a las personas que directamente o indirectamente me han ayudado en la obtención de este logro.

A Nelly, Crisitina y Marité porque desde mi llegada a esta facultad en 1988, me brindaron su amistad y me dieron una posibilidad de trabajo.

Al Dr. Toranzos por su dirección allá por 1994.

A Marisa por haber aceptado ser mi directora, por la forma natural y sabia con que supo guiarme, por brindarme su tiempo, por socorrerme desinteresadamente en tantos trámites y situaciones (señora), y por haberme permitido compartir su amistad con personas maravillosas como Celina, Marcia, Jayme y João, a quienes también agradezco su apoyo. 


\section{Contenido}

1 Introducción 1

2 Definiciones y Resultados Generales 5

2.1 Familias de conjuntos . . . . . . . . . . . . 5

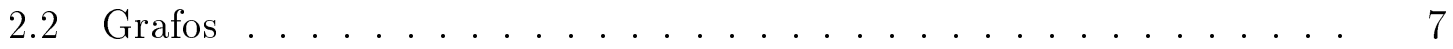

2.3 El Operador Intersección . . . . . . . . . . . . . . . . . . . 12

2.4 El Operador Clique . . . . . . . . . . . . . . . . . . . 13

$\begin{array}{lll}3 & K \text { (Planares) y } K^{-1} \text { (Planares) } & 17\end{array}$

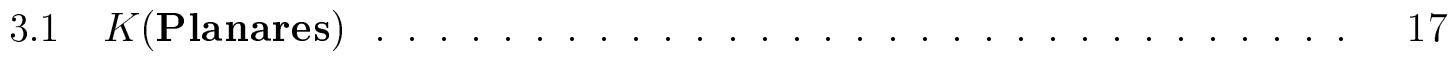

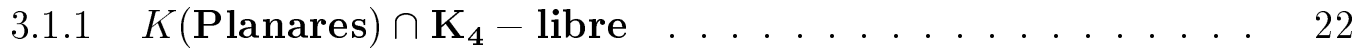

$3.1 .2 K$ (Planares) $\cap \mathbf{K}_{\mathbf{3}}-$ libre $\ldots \ldots \ldots \ldots \ldots$

3.1 .3 Ejemplos . . . . . . . . . . . . . . . . 31

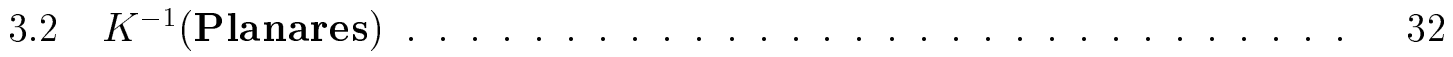

4 Grafos Planares Helly y 3-Helly 35

4.1 Introducción . . . . . . . . . . . . . . . . . 35

4.2 Las caracterizaciones . . . . . . . . . . . . . . 36

$5 \quad K($ Grafos $) \cap$ Planares $\quad 41$

5.1 Introducción . . . . . . . . . . . . . . . . . . 41

5.2 Triángulo extendido según una familia de completos . . . . . . . . 42

5.3 Condición necesaria para grafo clique planar . . . . . . . . . 48

5.4 Triángulos extendidos de un Grafo Planar que son Grafos Cliques . . 56

6 Asignación Triángulo-Vértice $\quad 67$

6.1 Definiciones . . . . . . . . . . . . . . . . 68

6.2 Una nueva caracterización de los Grafos Clique . . . . . . . 73

6.3 Ejemplos de Aplicación . . . . . . . . . . . . . . . . . 75 
Bibliografía 


\section{Capítulo 1}

\section{Introducción}

Se llama completo de un grafo a un conjunto de vértices adyacentes entre sí; si un completo es maximal con respecto a la inclusión, se dice que es un clique del grafo. Los cliques son estructuras especiales que naturalmente han despertado interés desde el mismo inicio de la Teoría de Grafos. Varios problemas famosos, como por ejemplo el problema de coloración de un grafo, o el problema de satisfabilidad de una fórmula lógica, se han vinculado y formulado en términos de los clique de un grafo. Por otro lado, exite una gama de problemas motivados en el propio estudio de los cliques de un grafo. Particularmente haremos foco en el estudio del grafo que muestra la relación de intersección entre estos clique: el grafo clique.

Dado un grafo $\mathbf{G}$ obtenemos el grafo clique de $\mathbf{G}$ considerando un vértice por cada clique de $\mathbf{G}$ y haciendo dos vértices adyacentes si los correspondientes clique tienen intersección no vacía.

De esta simple definición surgen inmediatamente varias preguntas; las siguientes tres son las que han dado origen a las tres principales líneas de investigación:

¿Todo grafo es el grafo clique de algún grafo?.

Dada una clase particular de grafos, ¿cómo es la clase formada por los grafos clique de los grafos dados?.

El proceso, que partiendo de un grafo dado obtiene iterativamente el grafo clique del 
grafo clique, ¿̇es convergente o genera una secuencia infinita de distintos grafos?.

En relación con la primer pregunta el artículo de más vieja data es el de Hamelink [14] donde se muestra que no todo grafo es grafo clique, y se da una condición suficiente para que un grafo sea grafo clique: que la familia de sus cliques tenga la propiedad de Helly (toda subfamilia mutamente intersectante tiene intersección no vacía). A los grafos que satisfacen esta condición les llamaremos grafos Helly.

Posteriormente Robert y Spencer [22], continuando con las ideas de Hamelink, encuentran una condición necesaria y suficiente para que un grafo sea grafo clique: que exista una familia de completos (no necesariamente los cliques) que cubra las arista del grafo y que tenga la propiedad Helly. A tales familias le llamaremos familias $\mathcal{R}-\mathcal{S}$. Esta caracterización de los grafos clique, la única conocida, no ha podido ser implementada en un algoritmo polinomial por lo que el problema de la complejidad del reconocimiento de los grafos clique continúa abierto.

Siguiendo esta línea de trabajo, en [10] se desarrolla un algoritmo no polinomial para decidir si un grafo es grafo clique o no; y en [12], se prueba que el problema de reconocimiento de los grafos clique puede reducirse al estudio de los grafos de diámetro 2. En [11] se presenta una forma de obtener a partir de una familia $\mathcal{R}-\mathcal{S}$ de un grafo $\mathbf{G}$, otro grafo tal que su grafo clique sea $\mathbf{G}$.

La rama de investigación surgida a partir de la segunda pregunta es más reciente.

Si llamamos $K$ al operador que a cada grafo le hace corresponder su grafo clique, se trata del estudio de la imagen por el operador $K$ de determinadas clases de grafos. En distintos artículos se ha caracterizado la imagen de las clases: Helly [8], Cordales y UV [27], DV y RDV [20], entre otras.

En [9] se engloba varios de los artículos antes mencionados, mostrando que el comportamiento del operador clique es uniforme en ciertas clases de grafos de intersección.

En respuesta a la tercer pregunta, el estudio de la convergencia del operador clique, han surgido publicaciones como [5], [6], [7], [15], [16], [19], entre muchas otras.

Para una visión actualizada, general pero detallada, de los prinicipales resultados obtenidos en las distintas líneas de investigación, y de la bibliografía existente, el 
lector interesado puede consultar [26].

Una muestra tanto de la importancia y actualidad como de la producción en torno al estudio de los cliques de un grafo, es la realización del I Latinamerican Workshop on Cliques of Graphs (Rio de Janeiro, Abril 2002). Se espera que esta reunión científica se desarrolle con cierta periodicidad en distintas ciudades de Latinoamérica.

El presente trabajo se relaciona con la primera y segunda pregunta anteriormente planteadas. Buscamos caracterizar los grafos clique en general, y para esto ahondamos en el estudio de los grafos clique de una clase particular: los grafos planares; y especialmente buscamos características de los grafos planares que son grafos clique. ¿Porqué los grafos planares?. Por un lado, aunque en el contexto del estudio de la convergencia del operador, la investigacion del comportamiento del operador clique sobre la clase de los planares fue sugerida por Prisner en [19]. Por otro lado, consideramos que los grafos planares podían resultar accesibles dado que sus cliques contienen a lo sumo cuatro vértices, pero principalmente porque conocíamos caracterizaciones de los grafos planares como grafos de intersección [24], con lo cual esperábamos aplicar los resultados de [9]. Paradójicamente, no fue de allí que surgieron los resultados que incluimos en esta Tesis. Mas, la táctica dió sus frutos: en base a lo observado sobre los grafo clique de los grafo planares y sobre los grafos planares que son grafos clique, logramos conclusiones sobre los grafos clique en general y obtuvimos una nueva caraterización de estos grafos.

Esta Tesis está organizada de la siguiente forma:

En el Capítulo 2 damos definiciones básicas y resultados generales sobre familias de conjuntos, grafos, y operadores.

En el Capítulo 3 estudiamos la imagen directa y la imagen inversa por el operador $K$, de la clase de los grafos planares. En el primer caso obtendremos distintas condiciones necesarias para que un grafo sea grafo clique de un grafo planar, y caracterizaremos totalmente los grafos $\mathbf{K}_{3}$ - libre y $\mathbf{K}_{4}$ - libre en estas condiciones. Si bien queda abierto el problema de caracterizar totalmente la clase $K($ Planares), la resolución del caso de los grafos $\mathbf{K}_{4}$ - libre y de los grafos $\mathbf{K}_{3}$ - libre, nos da una idea de la 
complejidad del problema planteado.

En el segundo caso probaremos que determinar si el grafo clique de un grafo dado es planar, puede hacerse eficientemente.

En el Capítulo 4 damos una caracterización de los grafos planares Helly y de los 3-Helly, mediante una sencilla descripción de los triángulos extendidos de estos grafos. En el Capítulo 5 generalizamos la noción de triángulo extendido y obtenemos un marco común para los resultados de Szwarcfiter (que caracterizan a los grafos Helly) y de Roberts-Spencer (que caracterizan a los grafos clique).

Damos una condición necesaria para que un grafo planar sea grafo clique y caracterizamos totalmente los triángulos extendidos de un grafo planar que son grafos clique. Dejamos abierta una posibilidad de caracterización total de los grafos clique planares mediante la compatibilidad de los cubrimientos de sus triángulos extendidos.

En el Capítulo 6 mostramos que si a cada triángulo del grafo se le puede asignar un vértice, respetando ciertas condiciones, es posible construir a partir de estos vértices una familia $\mathcal{R}-\mathcal{S}$ del grafo y viceversa. Obtenemos así una nueva caracterización de los grafos cliques.

Finalmente presentamos ejemplos de aplicación de la nueva caracterización de los grafos clique, mostrando hacia donde se encaminan nuestros próximos trabajos.

Vale mencionar que de los resultados presentados en esta Tesis surgieron las publicaciones [1], [2], [3], y [4]. 


\section{Capítulo 2}

\section{Definiciones y Resultados Generales}

En este capítulo damos definiciones básicas y resultados generales sobre familias de conjuntos, grafos, operadores, que utilizaremos a lo largo de este trabajo y que son de uso corriente en Teoría de Grafos. El objetivo del mismo es presentar claramente, para comodidad del lector, la notación a utilizar y los resultados que damos por conocidos. No incluimos las demostraciones de éstos ya que figuran en la bibliografía citada.

\subsection{Familias de conjuntos}

Sea $I$ un conjunto finito y no vacío. Una familia de conjuntos con subíndices en $I$ es una secuencia finita de conjuntos finitos y no vacíos, $\mathcal{F}=\left(F_{i}\right)_{i \in I}$. Los $\underline{\text { miembros }}$ de la familia son los conjuntos $F_{i}$. Los elementos de la familia son los elementos del conjunto $\cup \mathcal{F}=\bigcup_{i \in I} F_{i}$, es decir son los elementos de los conjuntos $F_{i}$, miembros de $\mathcal{F}$.

Sean $\mathcal{F}=\left(F_{i}\right)_{i \in I}$ y $\mathcal{M}=\left(M_{j}\right)_{j \in J}$ familias de conjuntos. Decimos que $\mathcal{F}$ está debajo de $\mathcal{M}$, y lo notamos $\mathcal{F} \preceq \mathcal{M}$, si existe una aplicación $\alpha: I \rightarrow J$ tal que para todo $i \in I$ se verifica que $F_{i} \subseteq M_{\alpha(i)}$. 
Decimos que $\mathcal{F}$ está estrictamente debajo de $\mathcal{M}$, y lo notamos $\mathcal{F} \prec \mathcal{M}$, si existe una aplicación $\alpha: I \rightarrow J$, inyectiva, tal que para todo $i \in I$ se verifica que $F_{i} \subseteq M_{\alpha(i)}$.

Decimos que $\mathcal{F}$ es una subfamilia de $\mathcal{M}$, y lo notamos $\mathcal{F} \subseteq \mathcal{M}$, si existe una aplicación $\alpha: I \rightarrow J$, inyectiva, tal que para todo $i \in I$ se verifica que $F_{i}=M_{\alpha(i)}$. Es claro que si $J^{\prime} \subseteq J$ entonces $\mathcal{M}^{\prime}=\left(M_{j}\right)_{j \in J^{\prime}}$ es una subfamilia de $\mathcal{M}$.

Es fácil ver que $\mathcal{F} \subseteq \mathcal{M}$ implica $\mathcal{F} \prec \mathcal{M}$ y que $\mathcal{F} \prec \mathcal{M}$ implica $\mathcal{F} \preceq \mathcal{M}$, pero en general no valen las implicaciones recíprocas.

Por otra parte, $\mathcal{F} \cup \mathcal{M}$ indica la familia cuyos miembros son los miembros de $\mathcal{F}$ y los miembros de $\mathcal{M}$.

Sean $u$ y $v$ elementos de la familia de conjuntos $\mathcal{F}=\left(F_{i}\right)_{i \in I}$, decimos que u está separado de $v$ en $\mathcal{F}$, si existe $i \in I$ tal que $u \in F_{i}$ y $v \notin F_{i}$. Es fácil ver que

$$
u \text { está separado de } v \text { en } \mathcal{F} \Longleftrightarrow v \notin \bigcap_{i \in I / u \in F_{i}} F_{i}
$$

Si $u$ no está separado de $v$ en $\mathcal{F}$, decimos que $u$ está $\underline{\text { dominado }}$ por $v$ en $\mathcal{F}$ o bien que $v$ domina a $u$. Los elementos $u$ y $v$ están separados si $u$ está separado de $v$ y $v$ está separado de $u$. La familia $\mathcal{F}$ se dice separadora si todo par de elementos está separado, o en otras palabras $\mathcal{F}$ es separadora si y solo si no hay en $\mathcal{F}$ elementos dominados.

Son equivalentes

$$
\begin{array}{r}
\mathcal{F}=\left(F_{i}\right)_{i \in I} \text { separadora } \\
\forall u, v \in \cup \mathcal{F}, \exists i \in I \text { tal que } u \in F_{i} \text { y } v \notin F_{i} \\
\forall u \in \cup \mathcal{F},\{u\}=\bigcap_{i \in I / u \in F_{i}} F_{i} .
\end{array}
$$

Una familia de conjuntos es mutuamente intersectante si todo par de miembros de la familia tienen intersección no vacía. La intersección total o intersección de una familia $\mathcal{F}$ de conjuntos, es el conjunto intersección de todos sus miembros, el cual denotaremos mediante $\cap \mathcal{F}$. 
Se dice que una familia de conjuntos tiene la propiedad de Helly o bien que es una

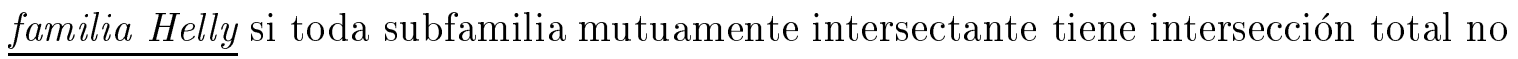
vacía. Análogamente dado $k \in \mathbf{N}$, una familia de conjuntos se dice $\underline{k \text {-Helly }}$ si toda subfamilia mutuamente intersectante, con a lo sumo $k$ miembros, tiene intersección total no vacía.

Proposición 2.1.1. [22] Una familia de conjuntos tiene la propiedad de Helly si y solo si dados tres elementos cualesquiera de la familia, la intersección de todos los miembros de la familia que contienen al menos dos de los tres elementos dados, es no vacía.

\subsection{Grafos}

Si $V$ es un conjunto no vacío $V^{[2]}$ denota el conjunto de todos los subconjuntos de $V$ que contienen exactamente dos elementos. Un grafo $\mathbf{G}$ es un par ordenado $(V(\mathbf{G}), E(\mathbf{G}))$ donde $V(\mathbf{G})$ es un conjunto finito, no vacío, cuyos elementos llamamos

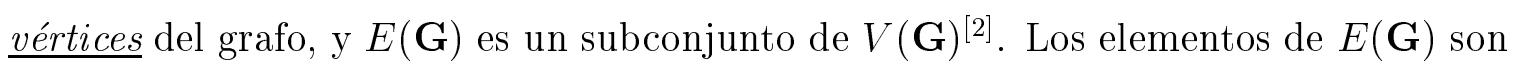
las aristas del grafo. Grafos denota al conjunto de todos los grafos.

En general indicamos con $n$ a la cantidad de vértices del grafo y con $m$ a la cantidad de aristas: $n=|V(\mathbf{G})|, m=|E(\mathbf{G})|$.

Dos grafos $\mathbf{G}$ y $\mathbf{G}^{\prime}$ son isomorfos si existe una aplicación $\psi: V(\mathbf{G}) \longrightarrow V\left(\mathbf{G}^{\prime}\right)$, biyectiva, tal que $E\left(\mathbf{G}^{\prime}\right)=\{\{\psi(u), \psi(v)\},\{u, v\} \in E(\mathbf{G})\}$. Es fácil ver que la relación binaria entre grafos definida por "es isomorfo a", es una relación de equivalencia. Salvo que se haga mención en contrario, a lo largo de este trabajo consideraremos como iguales a grafos isomorfos.

Decimos que dos vértices, $u$ y $v$, son adyacentes o vecinos en $\mathbf{G}$ y notamos $u \sim_{\mathbf{G}} v$, si $\{u, v\} \in E(\mathbf{G})$. Si dos vértices de $\mathbf{G}$ no son adyacentes notamos $u \chi_{\mathbf{G}} v$. En lo que sigue una arista $e=\{u, v\}$, se denotará $u v ; u$ y $v$ se dicen los vértices extremos de $e$. Una arista $e$ es $\underline{\text { incidente }}$ en un vértice $v$, si $v$ es un vértice extremo de $e$. Si $v$ es un vértice de $\mathbf{G}$ el entorno abierto de $v$ en $\mathbf{G}$ es el conjunto de vértices de $\mathbf{G}$ 
adyacentes a $v$.

$$
N_{\mathbf{G}}(v)=\left\{w \in V(\mathbf{G}) / w \sim_{\mathbf{G}} v\right\}
$$

El entorno cerrado de $v$ incluye además al vértice $v$.

$$
N_{\mathbf{G}}[v]=N_{\mathbf{G}}(v) \cup\{v\} .
$$

Un vértice $v$ se dice $\underline{\text { aislado }}$ si $N_{\mathbf{G}}(v)=\emptyset$.

Un vértice $v$ se dice $\underline{\text { universal }}$ de $\mathbf{G}$ si $N_{\mathbf{G}}[v]=V(\mathbf{G})$.

El $\underline{\text { grado }}$ en $\mathbf{G}$ de un vértice $v$ es el número de aristas de $E(\mathbf{G})$ incidentes en él.

$$
d_{\mathbf{G}}(v)=\left|N_{\mathbf{G}}(v)\right|
$$

En todos los casos anteriores si el contexto es claro puede omitirse el subíndice $\mathbf{G}$.

Decimos que $\mathbf{G}^{\prime}=\left(V\left(\mathbf{G}^{\prime}\right), E\left(\mathbf{G}^{\prime}\right)\right)$ es un subgrafo de $\mathbf{G}=(V(\mathbf{G}), E(\mathbf{G}))$ cuando,

$$
V\left(\mathbf{G}^{\prime}\right) \subseteq V(\mathbf{G}) \text { y } E\left(\mathbf{G}^{\prime}\right) \subseteq E(\mathbf{G})
$$

Si $V^{\prime} \subseteq V(\mathbf{G})$, el subgrafo de $\mathbf{G}$ inducido por $V^{\prime}$ es el grafo $\mathbf{G}\left[V^{\prime}\right]$ tal que

$$
V\left(\mathbf{G}\left[V^{\prime}\right]\right)=V^{\prime} \quad E\left(\mathbf{G}\left[V^{\prime}\right]\right)=E(\mathbf{G}) \cap V^{\prime[2]} .
$$

Si $E^{\prime} \subseteq E(\mathbf{G})$, el subrafo de $\mathbf{G}$ inducido por $E^{\prime}$ es el grafo $\mathbf{G}\left[E^{\prime}\right]$ tal que

$$
V\left(\mathbf{G}\left[E^{\prime}\right]\right)=\bigcup_{e \in E^{\prime}} e \quad E\left(\mathbf{G}\left[E^{\prime}\right]\right)=E^{\prime}
$$

$\mathbf{G}^{\prime}$ se dice subgrafo inducido de $\mathbf{G}$, si $\mathbf{G}^{\prime}=\mathbf{G}\left[V\left(\mathbf{G}^{\prime}\right)\right]$.

Usaremos la notación $\mathbf{G}^{\prime} \leq \mathbf{G}$ y $\mathbf{G}^{\prime} \subseteq \mathbf{G}$ para indicar que $\mathbf{G}^{\prime}$ es un subgrafo y, respectivamente, un subgrafo inducido de $\mathbf{G}$. 
Si $V^{\prime} \subseteq V(\mathbf{G}), \mathbf{G}-V^{\prime}$ es el grafo que se obtiene quitando a $\mathbf{G}$ los vértices pertenecientes a $V^{\prime}$ y las aristas incidentes en estos vértices.

$$
\mathbf{G}-V^{\prime}=\mathbf{G}\left[V(\mathbf{G})-V^{\prime}\right]
$$

Si $E^{\prime} \subseteq E(\mathbf{G}), \mathbf{G}-E^{\prime}$ es el grafo que se obtiene quitando a $\mathbf{G}$ las aristas pertenecientes a $E^{\prime}$, pero no sus vértices extremos.

$$
V\left(\mathbf{G}-V^{\prime}\right)=V(\mathbf{G}) \quad E\left(\mathbf{G}-E^{\prime}\right)=E(\mathbf{G})-E^{\prime}
$$

$\mathbf{G}_{v}$ es el subgrafo entorno de $v$ en $\mathbf{G}$, definido como el subgrafo de $\mathbf{G}$ inducido por el entorno abierto de $v$ en $\mathbf{G}$.

$$
\mathbf{G}_{v}=\mathbf{G}\left[N_{\mathbf{G}}(v)\right]
$$

Sean $\mathbf{G}$ y $\mathbf{G}^{\prime}$ grafos. El grafo unión de $\mathbf{G}$ y $\mathbf{G}^{\prime}$ es el grafo $\mathbf{G} \cup \mathbf{G}^{\prime}$ tal que

$$
V\left(\mathbf{G} \cup \mathbf{G}^{\prime}\right)=V(\mathbf{G}) \cup V\left(\mathbf{G}^{\prime}\right) \text { y } \quad E\left(\mathbf{G} \cup \mathbf{G}^{\prime}\right)=E(\mathbf{G}) \cup E\left(\mathbf{G}^{\prime}\right) .
$$

Un grafo $\mathbf{G}$ se dice completo si dos cualesquiera de sus vértices son adyacentes, es decir si $E(\mathbf{G})=V(\mathbf{G})^{[2]}$. Un subgrafo completo es un subgrafo que es un grafo completo. Un completo de un grafo $\mathbf{G}$ es un subconjunto $C$ de $V(\mathbf{G})$ tal que $\mathbf{G}[C]$ es un subgrafo completo. El tamaño de un completo es su cardinal. Diremos que un completo contiene a una arista, si los vértices extremos de la arista pertenecen al completo. Si C es un completo, el correspondiente subgrafo completo $\mathrm{G}[C]$ se denotará $\mathbf{C}$. Un triángulo de un grafo $\mathbf{G}$ es un completo de $\mathbf{G}$ con tres vértices. $T(\mathbf{G})$ denotará el conjunto de todos los triángulo de $\mathbf{G}$. Un clique de $\mathbf{G}$ es un completo de $\mathbf{G}$, maximal respecto a la relación de inclusión de conjuntos. Mientras el contexto sea claro y no se preste a confusión llamaremos también completo a un subgrafo completo, y clique a un subgrafo cuyo conjunto de vértices es un clique. 
La familia de cliques de $\mathbf{G}$ se notará $\mathcal{C}(\mathbf{G})$. Los elementos de esta familia son los vértices de $\mathbf{G}$, decimos entonces que el vértice $u$ está separado (dominado) del vértice $v$, si $u$ está separado (dominado) del vértice $v$ en $\mathcal{C}(\mathbf{G})$. Resulta entonces que $u$ es un vértice dominado si existe otro vértice $v$ tal que,

$$
v \in \bigcap_{C \in \mathcal{C}(\mathbf{G}), u \in C} C
$$

Un conjunto independiente o conjunto estable de $\mathbf{G}$ es un subconjunto de vértices de G, tales que dos cualesquiera de ellos no son adyacentes entre sí. El tamaño de un conjunto estable es la cantidad de vértices que posee.

$\omega(\mathbf{G})$ indica el tamaño del clique máximo de $\mathbf{G}$ y $\alpha(\mathbf{G})$ el tamaño del conjunto independiente máximo de $\mathbf{G}$.

Dado $k \in \mathbf{N}$, simbolizamos mediante $\mathbf{K}_{k}$ al grafo completo con $k$ vértices. Un grafo se dice $\mathbf{K}_{k}$ - libre si ninguno de sus subgrafos es isomorfo a $\mathbf{K}_{k}$. En general un grafo se dice $\underline{\mathbf{H}-\text { libre }}$ si ninguno de sus subgrafos inducidos es isomorfo a $\mathbf{H}$. Llamaremos $\mathbf{K}_{\mathbf{k}}$ - libre a la clase o conjunto de todos los grafos que son $\mathbf{K}_{k}-$ libre.

Un grafo $\mathbf{G}$ se dice bipartido si $V(\mathbf{G})$ puede particionarse en dos conjuntos, $V_{1}$ y $V_{2}$, estables. El grafo se dice bipartido completo si para todo $u \in V_{1}$ y $v \in V_{2}$ se tiene que $u v \in E(\mathbf{G}) . \mathbf{K}_{s, t}$ indica el grafo bipartido completo tal que $V_{1}$ posee $s$ vértices y $V_{2}$ posee $t$ vértices.

Sean $u$ y $v$ vértices de un grafo $\mathbf{G}$ y $k \in \mathbf{N}$. Una cadena en $\mathbf{G}$, de longitud $k$, entre $u$ y $v$, es una secuencia de vértices de $\mathbf{G}: w_{0}, w_{1}, \ldots, w_{k}$, tal que $w_{0}=u, w_{k}=v$ y para todo $1 \leq i \leq k, w_{i-1}$ y $w_{i}$ son adyacentes en $\mathbf{G}$. $w_{0}$ y $w_{k}$ son los vértices extremos de la cadena, los demás vértices se llaman $\underline{\text { interiores. }}$. Si para todo $i \neq j, w_{i} \neq w_{j}$ la cadena se dice un camino.

Un grafo $\mathbf{G}$ se dice $\underline{\text { conexo }}$ si para todo par de vértices de $\mathbf{G}$, existe un camino en $\mathbf{G}$ que los conecta. Una componente conexa de un grafo es un subgrafo conexo maximal. Un vértice $v$ de $\mathbf{G}$ se dice $\underline{\text { de corte }}$ si el número de componentes conexas del grafo 
$\mathbf{G}-v$, es mayor al de $\mathbf{G}$. Un bloque del grafo $\mathbf{G}$ es un subgrafo de $\mathbf{G}$ conexo, sin vértices de corte, y maximal respecto a esta propiedad.

Dado un grafo $\mathbf{G}$ una representación de $\mathbf{G}$ en el plano Euclídeo, es la imagen en el plano de un par de aplicaciones inyectivas: una del conjunto de vértices de $\mathbf{G}$ en los puntos del plano y otra del conjunto de aristas de $\mathbf{G}$ en el conjunto de curvas del plano, tales que, si $u$ y $v$ son los vértices extremos de una arista $e$, entonces los puntos del plano correspondientes a $u$ y a $v$ por la primer aplicación son los puntos extremos de la curva correspondiente a la arista $e$ por la segunda aplicación. Un grafo es planar si admite una representación en el plano Euclídeo tal que las curvas correspondientes a dos aristas distintas no se cruzan salvo, tal vez, en sus puntos extremos, una tal representación se dice una representación plana de $\mathbf{G}$ o bien una inmersión de $\mathbf{G}$ en el plano. Un grafo plano es un grafo planar con una dada representación plana. Es claro que si $\mathbf{G}^{\prime}$ es isomorfo a un grafo planar $\mathbf{G}$, entonces $\mathbf{G}^{\prime}$ es planar y más aún toda representación plana de $\mathbf{G}$ es una representación plana de $\mathbf{G}^{\prime}$. Planares denota la clase de todos los grafos planares.

A pesar de lo complejo que puede parecer a priori el problema de determinar si un grafo dado es planar o no, Kuratowski dió en 1930 un teorema de caracterización de los grafos planares que ha dado lugar al reconocimiento lineal de estos grafos [17]. Dicho teorema se basa en la idea de subdivisión: Decimos que el grafo $\mathbf{G}^{\prime}$ se obtiene por subdivisión de la arista $e=u v$ del grafo $\mathbf{G}$, si $V\left(\mathbf{G}^{\prime}\right)=V(\mathbf{G}) \cup\left\{w_{e}\right\}$, donde $w_{e} \notin V(\mathbf{G})$, y $E\left(\mathbf{G}^{\prime}\right)=E(\mathbf{G})-\{u v\} \cup\left\{u w_{e}, w_{e} v\right\} . \mathbf{G}^{\prime}$ es una subdivisión de $\mathbf{G}$ si se puede obtener a partir de $\mathbf{G}$ por una secuencia finita de subdivisiones de aristas.

Teorema 2.2.1. (Kuratowski) Un grafo $\mathbf{G}$ es planar si y solo si no tiene como subgrafo una subsivisión de $\mathbf{K}_{5}$ ni de $\mathbf{K}_{3,3}$, (Figura 2.1).

Es claro que la planaridad es una propiedad hereditaria para subgrafos, y, por lo tanto, para subgrafos inducidos, es decir:

$$
\mathbf{G} \in \text { Planares y } \mathbf{G}^{\prime} \leq \mathbf{G} \Longrightarrow \mathbf{G}^{\prime} \in \text { Planares. }
$$




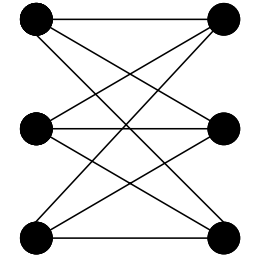

$\mathbf{K}$

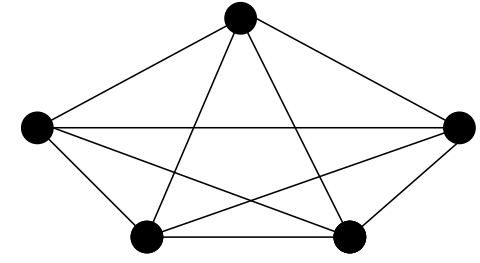

$\mathbf{K}_{5}$

Figura 2.1:

\subsection{El Operador Intersección}

Sea $\mathcal{F}=\left(F_{i}\right)_{i \in I}$ una familia de conjuntos, el grafo de intersección de $\mathcal{F}$, es un grafo que refleja la relación de intersección entre los miembros de la familia, es decir es un grafo que posee un vértice por cada miembro de la familia y dos vértices son adyacente si y solo si los correspondientes miembros tienen intersección no vacía. El operador intersección, que notamos $L$, hace corresponder a cada familia de conjuntos su grafo intersección. Formalmente definimos el grafo intersección $L(\mathcal{F})$ de la familia $\mathcal{F}=\left(F_{i}\right)_{i \in I}$ como el grafo cuyo conjunto de vértices es $I$, y dos vértices distintos $i$ y $j$ son adyacentes si y solo si $F_{i} \cap F_{j} \neq \emptyset$. Diremos que el vértice $i$ de $L(\mathcal{F})$ es correspondiente al miembro $F_{i}$ de $\mathcal{F}$ y recíprocamente. En la siguiente proposición resumimos resultados básicos ya conocidos sobre el operador intersección; por los mismos se puede consultar, por ejemplo, [13].

Proposición 2.3.1. $\quad \bullet \mathcal{F}^{\prime} \preceq \mathcal{F} \not L\left(\mathcal{F}^{\prime}\right) \leq L(\mathcal{F})$.

- $\mathcal{F}^{\prime} \subseteq \mathcal{F} \Longrightarrow L\left(\mathcal{F}^{\prime}\right) \subseteq L(\mathcal{F})$

- L es suryectivo pero no inyectivo.

- L restrigido a la clase de las familias Helly y separadoras es inyectivo. 


\subsection{El Operador Clique}

El grafo clique de $\mathbf{G}$ es el grafo de intersección de la familia de cliques de $\mathbf{G}$, es decir es el grafo $L(\mathcal{C}(\mathbf{G}))$.

El operador clique, que simbolizamos mediante $K$, hace corresponder a un grafo $\mathbf{G}$ $\mathrm{su}$ grafo clique, es decir

$$
K(\mathbf{G})=L(\mathcal{C}(\mathbf{G}))
$$

Cabe observar que como el grafo clique es grafo de intersección, tiene sentido hablar de la correspondencia entre los cliques de $\mathbf{G}$ y los vértices de $K(\mathbf{G})$.

Si Clase es una clase cualquiera de grafos, entonces $K$ (Clase) denotará la clase formada por los grafos clique de los grafos pertenecientes a Clase. Diremos que la clase Clase es fija para el operador clique si $K($ Clase $)=$ Clase.

Un grafo $\mathbf{G}$ se dice un grafo clique, si existe un grafo $\mathbf{H}$ tal que $K(\mathbf{H})=\mathbf{G}$; en otros términos, si $\mathbf{G}$ es el grafo clique de algún grafo $\mathbf{H}$.

$K$ (Grafos) indica la clase de los grafos cliques, es decir la imagen mediante el operador clique de la clase de todos los grafos.

Los siguientes son resultados básicos, ya conocidos, sobre el operador clique, [13].

Proposición 2.4.1. • $\mathbf{G}^{\prime} \leq \mathbf{G} \not K\left(\mathbf{G}^{\prime}\right) \leq K(\mathbf{G})$.

- $\mathbf{G}^{\prime} \subseteq \mathbf{G} \Longrightarrow K\left(\mathbf{G}^{\prime}\right) \leq K(\mathbf{G})$.

- K no es suryectivo , ni inyectivo.

- K restrigido a la clase de los grafos cuya familia de cliques es Helly y separadora es inyectivo.

Como hemos mencionado, el operador clique no es un operador suryectivo, es decir

$$
K(\text { Grafos }) \subset \text { Grafos pero } K(\text { Grafos }) \neq \text { Grafos }
$$

El ejemplo más sencillo de grafo que no es grafo clique es el de grafo $\mathbf{P}$, que llamaremos Pirámide, representado en la Figura 2.2. 


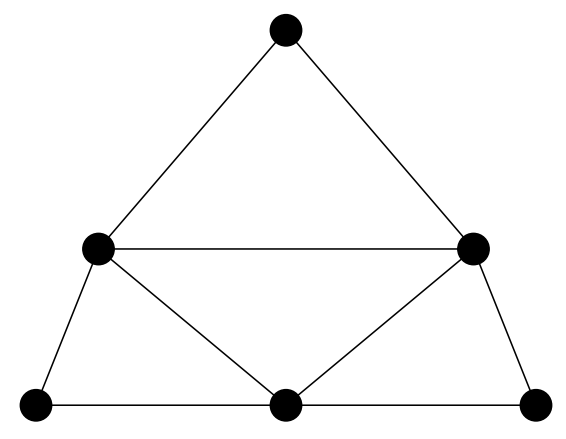

Figura 2.2: Grafo P.

Tiene sentido entonces hablar del problema de reconocimiento de los grafos cliques, que, en la forma clásica, se enuncia de la siguiente manera:

Entrada: Un grafo $\mathbf{G}=(V(\mathbf{G}), E(\mathbf{G}))$.

$\underline{\text { Pregunta: }}$ Existe un grafo $\mathbf{H}$ tal que $K(\mathbf{H})=\mathbf{G}$ ?

A la fecha no se conoce un algoritmo eficiente para la solución de este problema [26]. La caracterización de los grafos clique, dada por Roberts y Spencer, que citamos en el Teorema 2.4.1, es hasta ahora la única conocida y no ha permitido la elaboración de un algoritmo de reconocimiento que trabaje en una cantidad de tiempo que dependa polinomialmente de la cantidad de vértices del grafo ingresado. En adelante diremos simplemente algoritmo polinomial o eficiente.

Una familia de completos de un grafo $\mathbf{G}$ es una familia de conjuntos $\left(F_{i}\right)_{i \in I}$, tal que para todo $i \in I, F_{i}$ es un completo de $\mathbf{G}$. Diremos que la familia de completos $\left(F_{i}\right)_{i \in I}$,

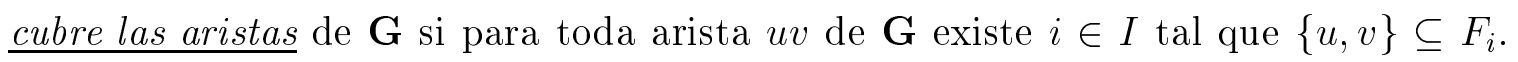
Un cubrimientos por completos de un grafo $\mathbf{G}$ es una familia de completos de $\mathbf{G}$ que cubre las aristas de $\mathbf{G}$. Una familia $\mathcal{R}-\mathcal{S}$ de un grafo $\mathbf{G}$ es una familia de completos de $\mathbf{G}$ que cubre sus aristas y que además tiene la propiedad de Helly. Una

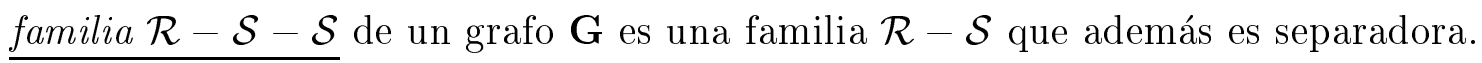
Diremos que un cubrimiento por completos, (una familia $\mathcal{R}-\mathcal{S}$, una familia $\mathcal{R}-\mathcal{S}-\mathcal{S}$ ), 
de un grafo $\mathbf{G}$ es minimal si ninguna subfamilia es un cubrimiento por completos (una familia $\mathcal{R}-\mathcal{S}$, una familia $\mathcal{R}-\mathcal{S}-\mathcal{S}$, respectivamente) de $\mathrm{G}$.

A continuación enunciamos el teorema debido Roberts y Spencer, que proporciona la caracterización de los grafos clique sobre la cual hemos hecho referencia.

Teorema 2.4.1. (Roberts-Spencer)[22] Un grafo $\mathbf{G}$ es un grafo clique si y solo si existe una familia $\mathcal{R}-\mathcal{S}$ de $\mathbf{G}$.

Un grafo $\mathbf{G}$ es un grafo Helly, si la familia de cliques de $\mathbf{G}, \mathcal{C}(\mathbf{G})$, tiene la propiedad de Helly. Sea Helly, la clase de todos los grafos Helly.

Para cualquier grafo $\mathbf{G}, \mathcal{C}(\mathbf{G})$ es un cubrimiento por completos de $\mathbf{G}$, es claro entonces que que si $\mathcal{C}(\mathbf{G})$ tiene la propiedad Helly, entonces $\mathcal{C}(\mathbf{G})$ es una familia $\mathcal{R}-\mathcal{S}$ de $\mathbf{G}$, por lo tanto Helly $\subseteq K$ (Grafos). Más aún, en [8], Escalante demuestra que Helly es una clase fija para el operador clique,

$$
\text { Helly }=K(\text { Helly }) \text {. }
$$

Por otra parte, Jayme Szwarcfiter da en [25], una caracterización de los grafos Helly (ver Teorema 4.1.1) que permite el reconocimiento eficiente de estos grafos.

Pertenecer a la clase Helly o a la clase $K$ (Grafos) no son propiedades hereditarias para subgrafos inducidos. Un sencillo contraejemplo es el grafo Helly que se obtiene agregando a la Pirámide (Figura 2.2) un vértice universal.

Es fácil probar los siguientes lemas, sobre los cuales volveremos en varias oportunidades:

Lema 2.4.2. [22] Si $C$ es un clique de $\mathbf{G}$ de tamaño menor o igual que 3 , y $\mathcal{F}$ es una familia $\mathcal{R}-\mathcal{S}$ de $\mathbf{G}$ entonces $C$ es un miembro de $\mathcal{F}$, por lo tanto,

$$
\text { si } \mathbf{G} \in \mathbf{K}_{\mathbf{4}}-\text { libre, } \mathbf{G} \in K(\text { Grafos }) \Longleftrightarrow \mathbf{G} \in \text { Helly }
$$

Lema 2.4.3. Vale que

$$
\mathbf{K}_{3}-\text { libre } \subseteq \text { Helly }=K(\text { Helly }) \subseteq K(\text { Grafos })
$$


Lema 2.4.4. Sea $\mathbf{G}$ un grafo y $E^{\prime}$ el conjunto de aristas de $\mathbf{G}$ cuyos vértices extremos conforman un clique de $\mathbf{G}$,

$$
\mathbf{G} \in K(\text { Grafos }) \leftrightarrow \mathbf{G}-E^{\prime} \in K(\text { Grafos })
$$

Se deduce de éste último lema que el estudio de los grafos clique puede limitarse al caso de los grafos cuyos cliques contienen al menos tres vértices.

Si $\mathbf{G}$ es un grafo, $K^{-1}(\mathbf{G})$ es la imagen inversa de $\mathbf{G}$ por el operador clique, es decir el conjunto de todos los grafos $\mathbf{H}$ tales que $K(\mathbf{H})=\mathbf{G}$. Si Clase es una clase de grafos $K^{-1}($ Clase $)=\left\{K^{-1}(\mathbf{G}), \mathbf{G} \in \mathbf{C l a s e}\right\}$.

Es claro que $\mathbf{G} \in K(\mathbf{G r a f o s})$ si y solo si $K^{-1}(\mathbf{G}) \neq \emptyset$, y por otra parte si $K^{-1}(\mathbf{G}) \neq \emptyset$ entonces $K^{-1}(\mathbf{G})$ contiene una cantidad infinita de grafos no isomorfos entre sí.

El siguiente resultado permite obtener los grafos en la imagen inversa por el operador $K$, de un grafo clique dado, a partir de ciertas familias $\mathcal{R}-\mathcal{S}$ del grafo en cuestión, las familias $\mathcal{R}-\mathcal{S}-\mathcal{S}$.

Teorema 2.4.5. [11] Sean $\mathbf{G}$ y $\mathbf{H}$ grafos. $\mathbf{G}=K(\mathbf{H})$ si y solo si existe $\mathcal{F}$, familia $\mathcal{R}-\mathcal{S}-\mathcal{S}$ de $\mathbf{G}$, tal que $\mathbf{H}=L(\mathcal{F})$. 


\section{Capítulo 3}

\section{$K($ Planares $)$ y $K^{-1}($ Planares $)$}

En este capítulo estudiamos la imagen directa y la imagen inversa por el operador $K$, de la clase de los grafos planares.

En el primer caso obtendremos distintas condiciones necesarias para que un grafo sea grafo clique de un grafo planar, i.e. para que el grafo pertenezca a $K$ (Planares), y caracterizaremos totalmente los grafos $\mathbf{K}_{3}$ - libre y $\mathbf{K}_{4}$ - libre en estas condiciones. Si bien queda abierto el problema de caracterizar totalmente la clase $K$ (Planares), la resolución del caso de los grafos $\mathbf{K}_{4}$ - libre y de los grafos $\mathbf{K}_{3}-$ libre, nos da una idea de la complejidad del problema planteado.

En el segundo caso probaremos que determinar si el grafo clique de un grafo dado es planar, puede hacerse eficientemente; en otras palabras veremos que la clase de grafos $K^{-1}$ (Planares) tiene reconocimiento polinomial.

\section{1 $K$ (Planares $)$}

Si $\mathbf{G} \in K($ Planares) y $v \in V(\mathbf{G})$, existe un grafo planar $\mathbf{H}$ tal que $\mathbf{G}=K(\mathbf{H})$ y un clique $C$ de $\mathbf{H}$ correspondiente a $v$. Como $\mathbf{H}$ es planar, $C$ tiene a lo sumo tamaño 4, entonces en $\mathbf{H}$ hay a lo sumo 4 cliques que intersectan a $C$ y son disjuntos entre si; por lo tanto en $\mathbf{G}$, el conjunto independiente máximo del subgrafo entorno de $v$, contiene a lo sumo cuatro vértices, es decir: 
Lema 3.1.1. Si $\mathbf{G} \in K($ Planares $) y v \in V(\mathbf{G})$, entonces $\alpha\left(\mathbf{G}_{v}\right) \leq 4$.

En forma más general este resultado se enuncia,

Lema 3.1.2. Sea $\mathbf{G}=K(\mathbf{H})$ y $v \in V(\mathbf{G})$. El tamaño de un conjunto independiente máximo en el subgrafo entorno de $v$ en $\mathbf{G}$ es menor o igual al tamaño del clique de $\mathbf{H}$ correspondiente a $v$.

El siguiente lema es equivalente al Lema 3.1.1 precedente.

Lema 3.1.3. Si $\mathbf{G} \in K(\mathbf{P l a n a r e s})$ entonces $\mathbf{G}$ es $\mathbf{K}_{1,5}-$ libre.

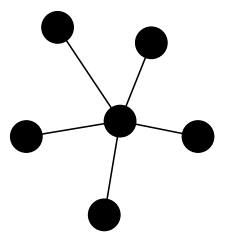

El grafo $\mathbf{K}_{1,5}$.

La recíproca de esta proposición no es en general cierta como se ve en el caso $\mathbf{G}=\mathbf{K}_{3,3}$, (Figura 2.1). Demostraremos que este grafo no está en $K($ Planares) (ver Subsección 3.1.3), sin embargo para todo vértice $v$ de $\mathbf{K}_{3,3}, \alpha\left(\mathbf{G}_{v}\right)=3$.

Es bueno observar en este momento, a partir del caso $\mathbf{K}_{3,3}$, lo complejo que puede resultar determinar que un grafo dado no está en $K$ (Planares): $\mathbf{K}_{3,3}$ es un grafo clique pues es un grafo $\mathbf{K}_{3}$ - libre (Lema 2.4.3). Para saber si $\mathbf{K}_{3,3}$ pertenece a la clase $K$ (Planares), debemos investigar los grafos en su imagen inversa por el operador clique, para ver si alguno de ellos es planar. Obtener grafos en la imagen inversa por el operador $K$, puede hacerse, por ejemplo, mediante la técnica referida en el Teorema 2.4.5. En la Figura 3.1 vemos un grafo, que llamaremos $\mathbf{H}$, tal que $K(\mathbf{H})=\mathbf{K}_{3,3}$.

Este grafo $\mathbf{H}$, no es planar (se puede ver facilmente que $\mathbf{H}$ es una subdivisión de $\mathbf{K}_{5}$ ). Sin embargo esto no implica que $\mathbf{K}_{3,3} \notin K$ (Planares), pues en la imagen inversa por 


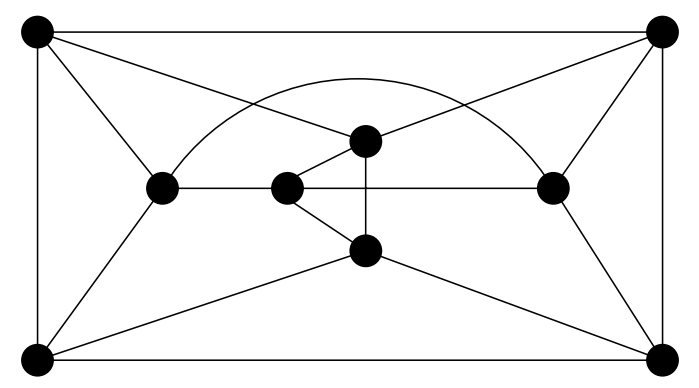

Figura 3.1: Grafo H.

$K$ de $\mathbf{K}_{3,3}$ hay además de $\mathbf{H}$ una cantidad infinita de grafos, no isomorfos a $\mathbf{H}$, alguno de los cuales podría ser planar, aún cuando $\mathbf{H}$ no lo sea.

Es claro que la propiedad de pertenecer a la clase de grafos $K$ (Planares), no es una propiedad hereditaria por subgrafos, ni por subgrafos inducidos, simplemente porque, como lo hemos observado en el capítulo anterior, la propiedad de pertenecer a la clase $K$ (Grafos) no lo es. Ahora, aunque un subgrafo inducido de un grafo clique puede no ser grafo clique, en el siguiente resultado veremos que un subgrafo inducido que satisface cierta condición es necesariamente grafo clique.

Si $\mathbf{G}=K(\mathbf{H})$ y $v \in V(\mathbf{G})$, notemos $C_{v}$ al clique de $\mathbf{H}$ correspondiente al vértice $v$.

Teorema 3.1.4. Sea $\mathbf{G}=K(\mathbf{H})$ y $\mathbf{G}^{\prime} \subseteq \mathbf{G}$. Llamemos $\mathbf{H}^{\prime}$ al subgrafo de $\mathbf{H}$,

$$
\mathbf{H}^{\prime}=\bigcup_{v \in V\left(\mathbf{G}^{\prime}\right)} \mathbf{C}_{v}
$$

Si para todo $v \in V(\mathbf{G})-V\left(\mathbf{G}^{\prime}\right),\left|N_{\mathbf{G}}(v) \bigcap V\left(\mathbf{G}^{\prime}\right)\right| \leq 2$, entonces $\mathbf{G}^{\prime}=K\left(\mathbf{H}^{\prime}\right)$.

Demostración: Basta probar que los cliques de $\mathbf{H}^{\prime}$ son exactamente los cliques de $\mathbf{H}$ correspondientes a los vértices de $\mathbf{G}^{\prime}$, es decir $\mathcal{C}\left(\mathbf{H}^{\prime}\right)=\left(C_{v}\right)_{v \in V\left(\mathbf{G}^{\prime}\right)}$.

Supongamos que $C$ es un clique de $\mathbf{H}^{\prime}$ distinto de $C_{v}$ para todo $v \in V\left(\mathbf{G}^{\prime}\right)$. Es claro que existe un clique de $\mathbf{H}$ que contiene a $C$, sea $v_{0}$ el vértice de $\mathbf{G}$ correspondiente a ese clique; resulta que $v_{0} \in V(\mathbf{G})-V\left(\mathbf{G}^{\prime}\right)$ y $C \subseteq C_{v_{0}}$ (Figura 3.2). 


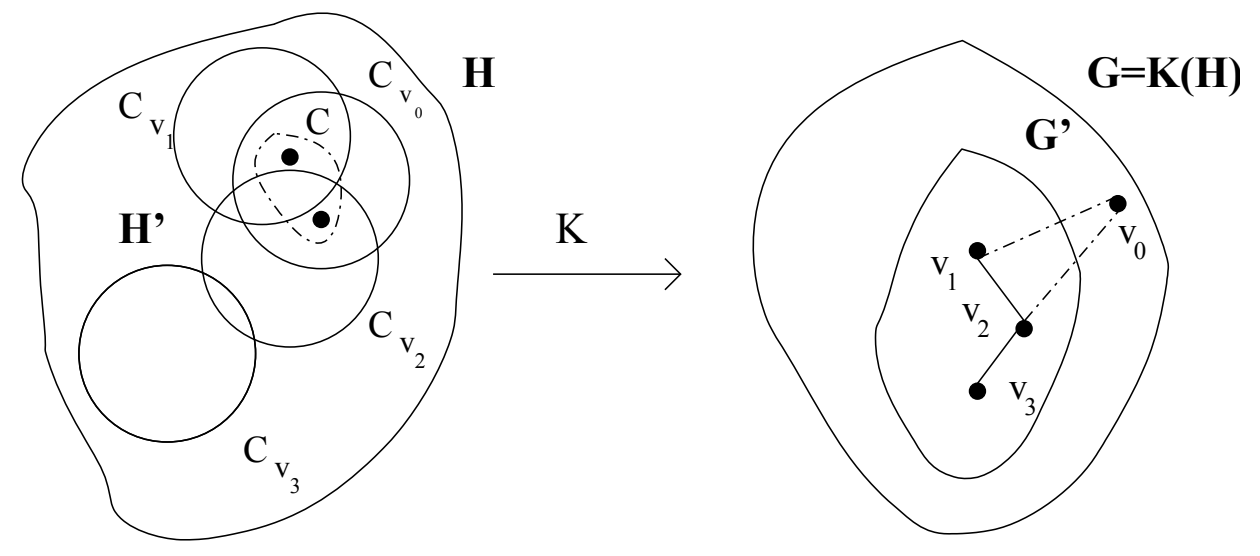

Figura 3.2:

Observemos que $\left\{v \in V\left(\mathbf{G}^{\prime}\right) / C_{v} \bigcap C \neq \emptyset\right\} \subseteq\left\{v \in V\left(\mathbf{G}^{\prime}\right) / C_{v} \bigcap C_{v_{0}} \neq \emptyset\right\}=\{v \in$ $\left.V\left(\mathbf{G}^{\prime}\right) / v \sim_{\mathbf{G}} v_{0}\right\}$. El primer conjunto tiene cardinal estrictamente mayor que uno, pues estamos suponiendo $C \neq C_{v}$ para todo $v \in \mathrm{G}^{\prime}$. El último conjunto tiene cardinal menor o igual que dos por hipótesis. Resulta que $\left|\left\{v \in V\left(\mathbf{G}^{\prime}\right) / C_{v} \bigcap C \neq \emptyset\right\}\right|=2$, pero esto es una contradicción, pues si $C$ intersecta a exactamente dos cliques, $C_{v_{1}}$ y $C_{v_{2}}$, y no está contenido en ninguno de ellos, deben existir vértices $x$ e $y$, tal que

$$
x \in C \cap\left(C_{v_{1}}-C_{v_{2}}\right), \quad y \in C \cap\left(C_{v_{2}}-C_{v_{1}}\right),
$$

pero como $x y$ es una arista de $C$ y por lo tanto de $\mathbf{H}^{\prime}$, debe existir, por definición de unión de grafos, $w \in V\left(\mathbf{G}^{\prime}\right)$ tal que $x y$ es una arista de $C_{w}$ y así $x$ e $y$ son vértices de $C_{w}$, y por lo tanto $C$ también intersecta $C_{w}$.

Por otra lado aunque la clase $K($ Grafos ) no puede ser caracterizada por subgrafos prohibidos, el teorema anterior permite encontrar ciertas estructuras prohibidas dentro de un grafo pertenciente a la clase $K$ (Grafos) y en particular dentro de un grafo perteneciente a la clase $K$ (Planares): si un grafo $\mathbf{G}$ tiene un subgrafo $\mathbf{G}^{\prime} \notin$ 
$K$ (Grafos) en las condiciones del enunciado, entonces $\mathbf{G} \notin K$ (Grafos). Además, como la planaridad es una propiedad hereditaria, resulta que si un grafo $\mathbf{G}$ tiene un subgrafo $\mathbf{G}^{\prime} \notin K$ (Planares) en las condiciones del teorema anterior, entonces $\mathbf{G} \notin K($ Planares $)$.

Por ejemplo, cualquier grafo que se obtiene a partir de un grafo que no es grafo clique, por el agregado sucesivo de vértices de grado dos, no es grafo clique. Observar el caso ilustrado en la siguiente figura a partir de la Pirámide.
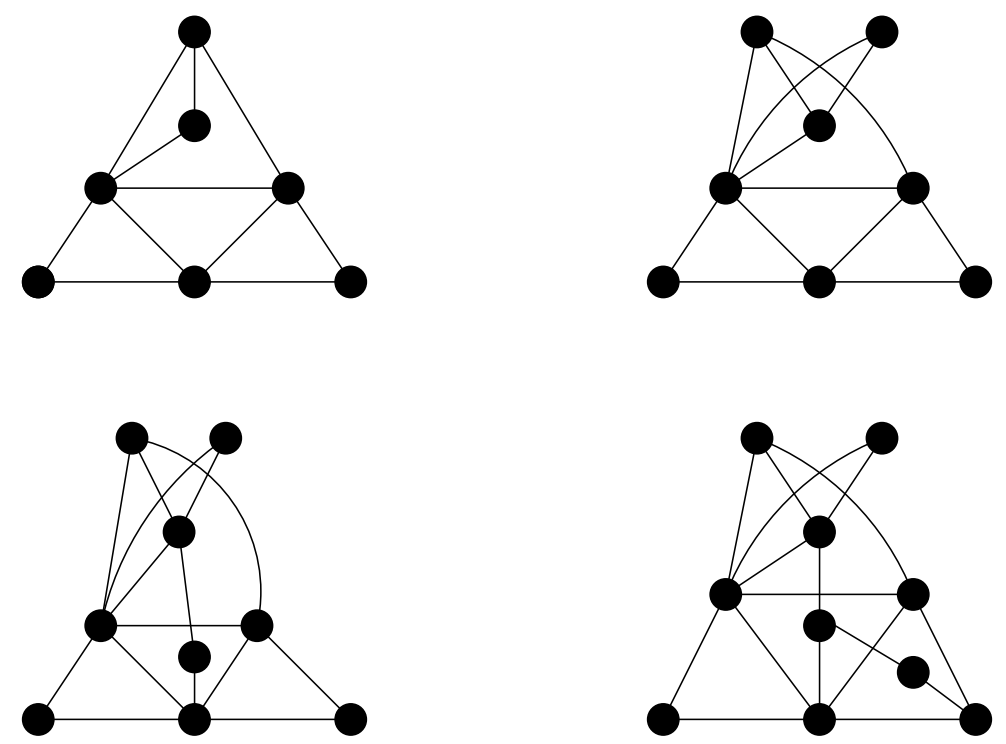

Grafos que no son grafos clique.

Corolario 3.1.5. Sea $\mathbf{G}=K(\mathbf{H})$ y $\mathbf{G}^{\prime}$ un bloque de $\mathbf{G}$, entonces $\mathbf{G}^{\prime}=K\left(\mathbf{H}^{\prime}\right)$ donde $\mathbf{H}^{\prime}=\bigcup_{v \in V\left(\mathbf{G}^{\prime}\right)} \mathbf{C}_{v}$.

Lema 3.1.6. Si $\mathbf{G} \in K($ Planares $)$ entonces cada bloque de $\mathbf{G}$ pertenece a $K(\mathbf{P l a n a r e s})$.

Demostración: Es inmediata a partir del Corolario 3.1.5 y del hecho de que la planaridad es una propiedad hereditaria.

La recíproca de este resultado no es, en general, cierta. Por ejemplo, vimos en el Lema 3.1.3 que $\mathbf{K}_{1,5}$ no está en $K$ (Planares), sin embargo cada bloque de $\mathbf{K}_{1,5}$ lo 
está. Ocurre en este ejemplo que el vértice de corte de $\mathbf{K}_{1,5}$, no satisface la condición del Lema 3.1.1, pero sí lo hace en cada uno de los bloques, esto se ve más generalmente en el siguiente resultado .

Lema 3.1.7. Sea $v$ un vértice de corte de un grafo $\mathbf{G}$ y $\mathbf{G}_{1}, \mathbf{G}_{2}, \ldots, \mathbf{G}_{k}$ los bloques de $\mathbf{G}$ a los que pertenece $v$. Entonces $\alpha\left(\mathbf{G}_{v}\right)=\sum_{1 \leq i \leq k} \alpha\left(\mathbf{G}_{i v}\right)$.

Demostración: Es inmediata. Destaquemos simplemente que $\mathbf{G}_{i v}$ es el subgrafo entorno de $v$ en $\mathbf{G}_{i}$.

Lema 3.1.8. Si $\mathbf{G} \in K(\mathbf{P l a n a r e s})$, v es un vértice de corte de $\mathbf{G} y \mathbf{G}_{1}, \mathbf{G}_{2}, \ldots, \mathbf{G}_{k}$ son los bloques de $\mathbf{G}$ a los que pertenece $v$, entonces $\sum_{1 \leq i \leq k} \alpha\left(\mathbf{G}_{i v}\right) \leq 4$.

Demostración: Es inmediata a partir del Lema 3.1.7 y del Lema 3.1.1.

Corolario 3.1.9. Si $\mathbf{G} \in K(\mathbf{P l a n a r e s})$, entonces todo vértice de corte de $\mathbf{G}$ está en a lo sumo 4 bloques de $\mathbf{G}$.

\subsection{1 $K($ Planares $) \cap \mathbf{K}_{4}-$ libre}

Buscamos caracterizar $K($ Planares $) \cap \mathbf{K}_{\mathbf{4}}$ - libre.

Los resultados obtenidos se desarrollaron a partir de las siguientes ideas básicas: de acuerdo al Teorema 2.4.5, $\mathbf{G} \in K($ Planares $)$ si y solo si existe una familia $\mathcal{R}-\mathcal{S}-\mathcal{S}$ de $\mathbf{G}$ tal que el grafo intersección de esta familia sea planar. Pensemos entonces en las familias $\mathcal{R}-\mathcal{S}-\mathcal{S}$ de un grafo $\mathbf{K}_{4}-$ libre.

Por el Lema 2.4.2, si $\mathbf{G}$ es $\mathbf{K}_{4}$ - libre y admite una familia $\mathcal{R}-\mathcal{S}$ entonces $\mathcal{C}(\mathbf{G})$ es subfamilia de cualquier familia $\mathcal{R}-\mathcal{S}$ de $\mathbf{G}$, es decir $\mathbf{G}$ tiene una única familia $\mathcal{R}-\mathcal{S}$ minimal: la familia de sus cliques. Las demás familias $\mathcal{R}-\mathcal{S}$ se obtienen agregando a la familia de los cliques miembros repetidos o bien miembros contenidos en otros miembros.

Algo similar ocurre respecto a la separación de la familia: si $\mathcal{C}(\mathbf{G})$ no es separadora, 
será necesario agregar miembros para que así sea.

Es claro que al agregar miembros, el grafo intersección de la familia se torna más complejo, con lo cual es de esperar que si en la imagen inversa por el operador clique hay algún grafo planar, este grafo planar sea el grafo intersección de la familia $\mathcal{R}-\mathcal{S}-\mathcal{S}$ de $\mathbf{G}$ formada por la familia de cliques de $\mathbf{G}$ con la menor cantidad posible de miembros agregados; es natural pensar que tal familia $\mathcal{R}-\mathcal{S}-\mathcal{S}$ es la familia formada por los cliques de $\mathbf{G}$ y un miembro $\{u\}$, por cada vértice $u \in V(\mathbf{G})$ dominado en $\mathbf{G}$.

Usaremos el siguiente lema en la demostración del teorema de caracterización.

Lema 3.1.10. Sea $\mathbf{G}$ un grafo y $\left(\mathbf{G}_{i}\right)_{i \in I}$ una familia finita de subgrafos conexos de G. El grafo $L(\mathcal{F})$, intersección de la familia $\mathcal{F}=\left(V\left(\mathbf{G}_{i}\right)\right)_{i \in I}$, es conexo si y solo si $\bigcup_{i \in I} \mathbf{G}_{i}$ es conexo.

Demostración: Para probar que $\bigcup_{i \in I} \mathbf{G}_{i}$ es conexo, consideremos $u$ y $v$ vértices de este grafo. Existen $\mathbf{G}_{i_{u}}, \mathbf{G}_{i_{v}}$ tales que $u \in V\left(\mathbf{G}_{i_{u}}\right)$ y $v \in V\left(\mathbf{G}_{i_{v}}\right)$. Sean $i_{u}$ e $i_{v}$ los correspondientes vértices de $L(\mathcal{F})$. Como $L(\mathcal{F})$ es conexo existe un camino $i_{0}, i_{1}, \ldots, i_{k}$ tal que $i_{0}=i_{u}$ e $i_{k}=i_{v}$. Como para todo $j, 1 \leq j \leq k, i_{j-1}$ es adyacente a $i_{j}$ en $L(\mathcal{F})$, resulta que los correspondientes conjuntos $V\left(\mathbf{G}_{j-1}\right)$ y $V\left(\mathbf{G}_{j}\right)$ de $\mathcal{F}$ tienen intersección no vacía, podemos entonces considerar vértices $w_{j}, 1 \leq j \leq k$, en estas intersecciones. Llamemos además $w_{0}=u$ y $w_{k+1}=v$. Así tenemos que para todo $j, 1 \leq j \leq k+1$, $w_{j-1}$ y $w_{j}$ pertenecen a $V\left(\mathbf{G}_{j}\right)$, como los subgrafos $\mathbf{G}_{j}$ son todos conexos, resulta que para todo $1 \leq j \leq k+1$ existe en $\bigcup_{i \in I} \mathbf{G}_{i}$ un camino entre $w_{j-1}$ y $w_{j}$ y por ende una cadena entre $w_{0}=u \mathrm{y} w_{k+1}=v$.

Para probar la implicación recíproca sean $i_{0}$ e $i_{0}^{\prime}$ vértices de $L(\mathcal{F})$; sean $V\left(\mathbf{G}_{i_{0}}\right)$ y $V\left(\mathbf{G}_{i_{0}^{\prime}}\right)$ los correspondientes miembros de la familia $\mathcal{F}$. Sean $u$ y $v$ vértices de $\bigcup_{i \in I} \mathbf{G}_{i}$ tal que $u \in V\left(\mathbf{G}_{i_{0}}\right)$ y $v \in V\left(\mathbf{G}_{i_{0}^{\prime}}\right)$. Como por hipótesis $\bigcup_{i \in I} \mathbf{G}_{i}$ es conexo existe un camino $v_{1}, v_{2}, \ldots v_{k}$ tal que $v_{1}=u$ y $v_{k}=v$. Como para todo $j, 1 \leq j \leq k-1$, la arista $e_{j}=v_{j} v_{j+1}$ es una arista de $\bigcup_{i \in I} \mathbf{G}_{i}$, existe un subgrafo $\mathbf{G}_{i_{j}}, i_{j} \in I$, tal que $e_{j} \in E\left(\mathbf{G}_{i_{j}}\right)$, (sin pérdida de generalidad se puede suponer $i_{1}=i_{0}$ e $i_{k-1}=i_{0}^{\prime}$ ), y por lo tanto $v_{j}$ y $v_{j+1}$ pertenecen a $V\left(\mathbf{G}_{i_{j}}\right)$, de donde $V\left(\mathbf{G}_{i_{j}}\right)$ y $V\left(\mathbf{G}_{i_{j+1}}\right)$ tienen 
intersección no vacía. Es claro entonces que los vértices de $L(\mathcal{F}), i_{j}$ correspondiente a los miembros $V\left(\mathbf{G}_{i_{j}}\right)$ conforman una cadena en $L(\mathcal{F})$, así tenemos una cadena entre $i_{1}=i_{0}$ e $i_{k-1}=i_{0}^{\prime}$.

Los grafos pertenecientes a la clase $K($ Planares $) \cap \mathbf{K}_{4}-$ libre son caracterizados por el siguiente teorema.

Teorema 3.1.11. Sea $\mathbf{G}$ grafo clique $\mathbf{K}_{4}$ - libre. $\mathbf{G} \in K(\mathbf{P l a n a r e s})$ si y solo si

1. $K(\mathbf{G})$ es un grafo planar.

2. Toda arista de $\mathbf{G}$ está en a lo sumo 3 cliques de $\mathbf{G}, y$

3. Si una arista uv de $\mathbf{G}$ está en 3 cliques de $\mathbf{G}:\{u, v, a\},\{u, v, b\} y\{u, v, c\}$, entonces los vértices $a, b$ y c no están en una misma componente conexa de $\mathbf{G}-T$, donde $T$ es el conjunto de aristas de los cliques de $\mathbf{G}$ que contienen a $u$ o $a v$.

Demostración: Sea $\mathbf{G} \in K($ Planares $) \cap \mathbf{K}_{4}$ - libre. Como $\mathbf{G} \in K($ Planares $)$, existe un grafo planar $\mathbf{H}$ tal que $\mathbf{G}=K(\mathbf{H})$. De acuerdo con el Teorema 2.4.5, existe $\mathcal{F}$, una familia $\mathcal{R}-S-S$ de $\mathbf{G}$ satisfaciendo $L(\mathcal{F})=\mathbf{H}$.

Como $\mathbf{G}$ es $\mathbf{K}_{4}$ - libre, por el Lema 2.4.2, $\mathcal{C}(\mathbf{G})$ es una subfamilia de $\mathcal{F}$, entonces $L(\mathcal{C}(\mathbf{G}))=K(\mathbf{G})$ es un subgrafo inducido de $L(\mathcal{F})=\mathbf{H}$ (Proposición 2.3.1). Con esto probamos que $K(\mathbf{G})$ es planar.

Supongamos que una arista $u v$ de $\mathbf{G}$ está en cuatro cliques diferentes. Como $\mathcal{F}$ es una familia separadora debe existir un miembro $F_{u}$ tal que $u \in F_{u}$ y $v \notin F_{u}$ (Equivalencias 2.1.2), claramente $F_{u}$ no es ninguno de los cuatro cliques mencionados. Como $F_{u}$ y los cuatro cliques son miembros de $\mathcal{F}$ y todos ellos son mutuamente intersectantes pues contienen a $u$, su intersección genera un $\mathbf{K}_{5}$ en $L(\mathcal{F})=\mathbf{H}$. Esto contradice la planaridad de $\mathbf{H}$. Hemos probado que toda arista de $\mathbf{G}$ está en a lo sumo 3 cliques. Probaremos la condición 3 por contradicción. Si 3 no es cierta, existe una arista $u v$ de $\mathbf{G}$ en tres cliques: $A=\{u, v, a\}, B=\{u, v, b\}$ y $C=\{u, v, c\}$; y se satisface que 


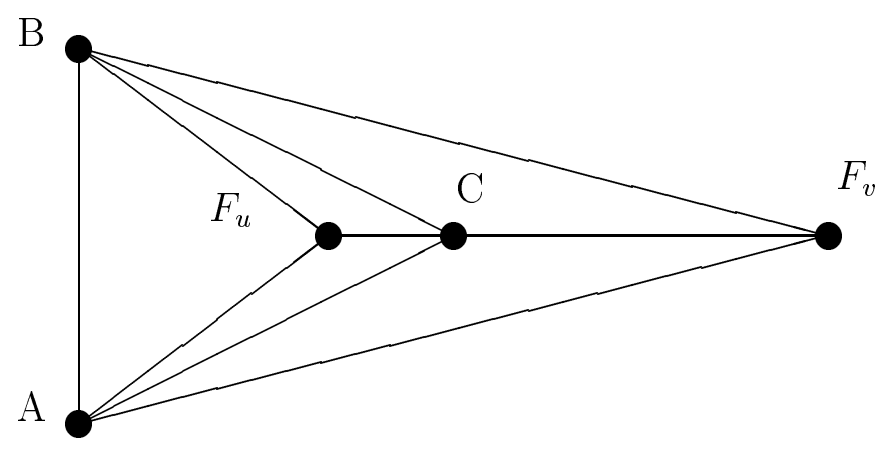

Figura 3.3: Subgrafo $\mathbf{S}$ de $\mathbf{H}=L(\mathcal{F})$.

los vértices $a, b$ y $c$ están en una misma componente conexa $\mathbf{X}$, de $\mathbf{G}-T$, donde $T$ es el conjunto de las aristas de los cliques de $\mathbf{G}$ que contienen $u$ o $v$.

Observemos que, por la propia definición de T,

$$
\text { Si } e \in E(\mathbf{G}-T) \text { y } e \in E\left(\mathbf{C}^{\prime}\right), C^{\prime} \text { clique de } \mathbf{G} \Longrightarrow u \notin C^{\prime} \text { y } v \notin C^{\prime} \text {. }
$$

Ahora, vimos que por ser $\mathcal{F}$ una familia separadora debe contener un miembro $F_{u}$ tal que $u \in F_{u}$ y $v \notin F_{u}$; por la misma razón debe existir otro miembro $F_{v}$ tal que $v \in F_{v}$ y $u \notin F_{v}$. La intersección de los cinco miembros de $\mathcal{F}: A, B, C, F_{u}$ y $F_{v}$ genera en $L(\mathcal{F})$ el subgrafo $\mathbf{S}$ de la Figura 3.3. $F_{u}$ y $F_{v}$ no pueden ser adyacentes en $\mathbf{H}$ por planaridad. Observar que llamamos en la misma forma a los miembros de $\mathcal{F}$ y a los correspondientes vértices de $L(\mathcal{F})$.

El subgrafo $\mathbf{S}$ es planar, pero demostraremos -a partir de él- que existe un subgrafo de $L(\mathcal{F})$,i.e. de $\mathbf{H}$, el cual no es planar pues contiene una subdivisión de $K_{3,3}$. Más precisamente, mostraremos un subgrafo $\mathbf{S}^{\prime}$ de $L(\mathcal{F})$, conexo y disjunto de $\mathbf{S}$ el cual tiene vértices adyacentes a $A, B$ y $C$, con lo cual habrá una subdivisión de $K_{3,3}$. Sea $\mathcal{C}_{\mathbf{X}}=\left(C_{i}\right)_{i \in I}$ la familia formada por todos aquellos cliques de $\mathbf{G}$ que contienen alguna arista de $\mathbf{X}$. Claramente $\mathcal{C}_{\mathbf{X}}$ es una subfamilia de $\mathcal{C}(\mathbf{G})$ y por lo tanto de $\mathcal{F}$, entonces $\mathbf{S}^{\prime}=L\left(\mathcal{C}_{\mathbf{X}}\right)$ es un subgrafo inducido de $L(\mathcal{F})$ (Proposición 2.3.1).

Si $C_{i}$ es un miembro de $\mathcal{C}_{\mathbf{X}}$, por definición de $\mathcal{C}_{\mathbf{X}}$ y la implicación 3.1.1, $C_{i}$ no contiene 
a $u$ ni a $v$, por lo tanto $C_{i}$ no es $A$, ni $B$, ni $C$, y tampoco es $F_{u}$ o $F_{v}$. Se deduce que $\mathbf{S}^{\prime}$ y $\mathbf{S}$ son subgrafos disjuntos, no tienen ningún vértice en común.

Por otra parte, como cada subgrafo $\mathbf{C}_{i}$ y $\bigcup_{i \in I} \mathbf{C}_{i}$ son conexos, por lo demostrado en el Lema 3.1.10, el subgrafo $\mathbf{S}^{\prime}=L\left(\mathcal{C}_{\mathbf{X}}\right)$ es conexo. Hemos demostrado que $\mathbf{S}^{\prime}$ es un subgrafo conexo de $L(\mathcal{F})$ disjunto de $\mathbf{S}$.

Ahora, como $a, b$ y $c$ son vértices de la componente conexa $\mathbf{X}$, existen aristas de $\mathbf{X}$ incidentes en esos vértices, por lo tanto hay un miembro de $\mathcal{C}_{\mathbf{X}}$ conteniendo a $a$, un miembro conteniendo a $b$ y un miembro conteniendo a $c$; pueden ser un mismo miembro o no. Claramente los vértices de $\mathbf{S}^{\prime}$ correspondientes a estos miembros son adyacentes en $L(\mathcal{F})$ a $A$, a $B$, y a $C$ respectivamente, tal como queríamos demostrar. Para demostrar la recíproca, consideremos un grafo clique $\mathbf{G}, \mathbf{K}_{4}$-libre. Por el Lema 2.4.2, $\mathbf{G}$ es un grafo Helly, por lo tanto $\mathcal{C}(\mathbf{G})$ es una familia $\mathcal{R}-S$ de $\mathbf{G}$. Llamemos $\operatorname{Dom}(\mathbf{G})=\left\{u_{1}, u_{2}, \ldots, u_{s}\right\}$ al conjunto de los vértices dominados de $\mathbf{G}$. En lo que sigue suponemos que $\operatorname{Dom}(\mathbf{G})$, es no vacío, en caso que lo fuese la demostración puede seguirse en forma análoga. Claramente la familia $\mathcal{F}=\mathcal{C}(\mathbf{G}) \bigcup\left(\left\{u_{i}\right\}\right)_{1 \leq i \leq s}$ tiene la propiedad de Helly pues $\mathcal{C}(\mathbf{G})$ la tiene y es separadora pues no tiene elementos dominados, por lo tanto es una familia $\mathcal{R}-S-S$ de $\mathbf{G}$. Concluimos por el Teorema 2.4.5 que $\mathbf{H}=L(\mathcal{F})$ es tal que $\mathbf{G}=K(\mathbf{H})$. Probaremos que si se satisfacen las condiciones 1, 2 y 3, $\mathbf{H}$ es un grafo planar y por lo tanto $\mathbf{G} \in K$ (Planares). Más precisamente, probaremos inductivamente que para todo $j, 0 \leq j \leq s$, el grafo $\mathbf{H}_{j}=L\left(\mathcal{F}_{j}\right)$ es planar, donde

$$
\begin{aligned}
\mathcal{F}_{0} & =\mathcal{C}(\mathbf{G}) \\
\mathcal{F}_{j} & =\mathcal{C}(\mathbf{G}) \bigcup\left(\left\{u_{i}\right\}\right)_{1 \leq i \leq j} \quad \text { cuando } j>0
\end{aligned}
$$

La proposición es verdadera cuando $j=0$, porque $\mathbf{H}_{0}=L\left(\mathcal{F}_{0}\right)=L(\mathcal{C}(\mathbf{G}))=K(\mathbf{G})$ que es planar por la hipótesis 1 . Suponiendo que para un dado $j=\ell-1 \geq 0, \mathbf{H}_{\ell-1}$ es planar, probaremos que $\mathbf{H}_{\ell}$ también lo es. Es claro que $\mathbf{H}_{\ell}$ es el grafo planar $\mathbf{H}_{\ell-1}$ con el agregado de un nuevo vértice $\left\{u_{\ell}\right\}$, y las aristas que lo hacen adyacente a 
aquellos vértices de $\mathbf{H}_{\ell-1}$ correspondientes a los cliques de $\mathbf{G}$ que contienen al vértice $u_{\ell}$. Veamos que tales cliques son a lo sumo tres.

Como $u_{\ell}$ es un vértice dominado de $\mathbf{G}$ debe existir en $\mathbf{G}$ un vértice $v$ que lo domina. Como $v$ domina a $u_{\ell}, v$ pertenece a todo clique que contiene a $u_{\ell}$ (Equivalencia 2.2.1); se deduce que la arista $v u_{\ell}$ está en todo clique que contiene a $u_{\ell}$, entonces, por la hipótesis $2, u_{\ell}$ no puede estar más que en tres cliques de G. Consideraremos por separado el caso en que $u_{\ell}$ está en uno o en dos cliques de $\mathbf{G}$ y el caso en que $u_{\ell}$ está en exactamente tres cliques.

Caso a: $u_{\ell}$ está en uno (dos) cliques de $\mathbf{G}$. Para obtener $\mathbf{H}_{\ell}$ debemos agregar a $\mathbf{H}_{\ell-1}$ el vértice $\left\{u_{\ell}\right\}$ y hacer este vértice adyacente a un único vértice (dos vértices que son extremos de una arista) de $\mathbf{H}_{\ell-1}$, entonces es claro que $\mathbf{H}_{\ell}$ es planar si $\mathbf{H}_{\ell-1}$ lo es. $\underline{\text { Caso b: }}$ : $u_{\ell}$ está en tres cliques de $\mathbf{G}$. Estos cliques deben ser $\mathbf{K}_{3}$, pues $\mathbf{G}$ es $\mathbf{K}_{4}$-libre. Sean estos cliques $A=\left\{u_{\ell}, v, a\right\}, B=\left\{u_{\ell}, v, b\right\}$ y $C=\left\{u_{\ell}, v, c\right\}$. Si $\mathbf{H}_{\ell-1}$ admite una representación plana tal que el triángulo con vértices $A, B$ y $C$ es la frontera de una cara, para obtener una representación de $\mathbf{H}_{\ell}$, podemos agregar a $\mathbf{H}_{\ell-1}$ el vértice $\left\{u_{\ell}\right\}$ en el centro de esta cara y hacerlo adyacente a $A, B$ y $C$ sin perder la planaridad, y la demostración del teorema está terminada.

Veamos que, en las condiciones del teorema, una tal representación siempre existe, pues de no ser así, se contradice la hipótesis 3; en otras palabras demostraremos que si no existe una representación de $\mathbf{H}_{\ell-1}$ como la dicha, los vértices $a, b$ y $c$ de $\mathbf{G}$ están en un subgrafo conexo de $\mathbf{G}-T$, donde $T$ es el conjunto de todas las aristas de los cliques de $\mathbf{G}$ que contienen a $u_{\ell}$ o a $v$.

Si $\mathbf{H}_{\ell-1}$ no admite una representación plana con el triángulo de vértices $A, B, C$ como frontera de una cara, es porque en $H_{\ell-1}$ hay subgrafos conexos, $\mathbf{S}$ y $\mathbf{S}^{\prime}$, disjuntos, que no contienen ninguno de los vértices $A, B$ o $C$, pero que contienen vértices adyacentes a $A, B$ y $C$, como se muestra en la Figura 3.4.

Veamos que uno de los dos subgrafos, $\mathbf{S}$ o $\mathbf{S}^{\prime}$ es tal que cada uno de sus vértices corresponde a un miembro de $\mathcal{F}_{\ell-1}$ que no contiene a $u_{\ell}$ ni a $v$; esto se deduce facilmente de los siguientes hechos: 


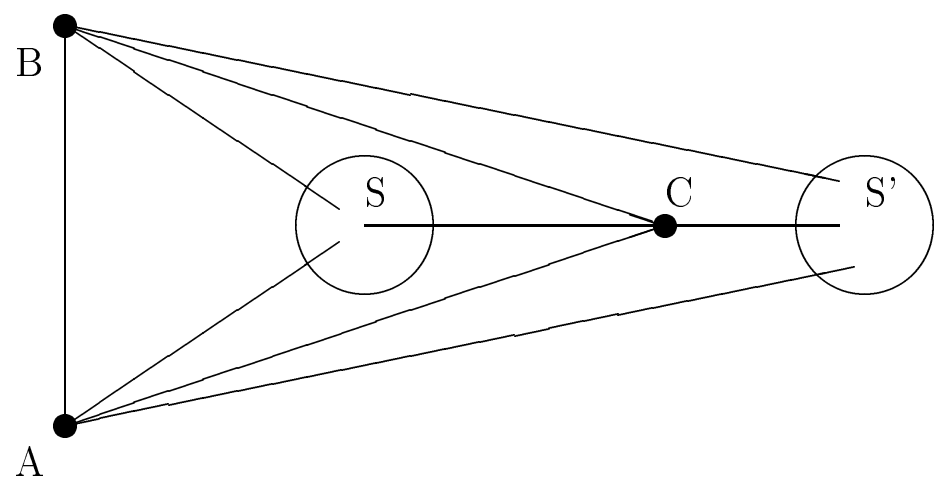

Figura 3.4: $\mathbf{H}_{\ell-1}=L\left(\mathcal{F}_{\ell-1}\right)$.

i) Los vértices de $\mathbf{S}$ y de $\mathbf{S}^{\prime}$ corresponden todos a miembros de $\mathcal{F}_{\ell-1}$ distintos de los cliques $A, B$ o $C$ (Ver Figura 3.4).

ii) Estamos suponiendo en el caso b) que los únicos miembros de $\mathcal{F}_{\ell-1}$ a los que pertenece $u_{\ell}$ son $A, B$ and $C$.

iii) $v$ es un elemento de la familia $\mathcal{F}_{\ell-1}$ que está en los miembros $A, B, C$ y tal vez en más pero, como por hipótesis inductiva, $L\left(\mathcal{F}_{\ell-1}\right)$ es planar, todo elemento de la familia está en a lo sumo cuatro miembros, de donde $v$ está en a lo sumo un miembro más, este miembro podría corresponder a un vértice de $\mathbf{S}$ o de $\mathbf{S}^{\prime}$, pero no de los dos. Podemos entonces asumir sin pérdida de generalidad que:

los vértices de $\mathbf{S}^{\prime}$ corresponden a miembros que no contienen a $u_{\ell}$ ni a $v$

Llamemos $\mathcal{F}^{\prime}$ a la subfamilia de $\mathcal{F}_{\ell-1}$ tal que $L\left(\mathcal{F}^{\prime}\right)=S^{\prime}$. Como cada miembro de la subfamilia $\mathcal{F}^{\prime}$ es el conjunto de vértices de un subgrafo conexo de $\mathbf{G}$, y como $\mathbf{S}^{\prime}=L\left(\mathcal{F}^{\prime}\right)$ es conexo, por el Lema 3.1.10, es conexo el subgrafo, que llamaremos $\mathbf{X}$, unión de los subgrafos de $\mathbf{G}$ cuyos conjuntos de vértices son los miembros de $\mathcal{F}_{\ell-1}$ correspondientes a los vértices de $\mathbf{S}^{\prime}$.

Veamos que $a, b$ y $c$ son vértices de $\mathbf{X}$. En $\mathbf{S}^{\prime}$ hay un vértice adyacente a $A=\left\{u_{\ell}, v, a\right\}$; por la afirmación 3.1.2 ese vértice debe corresponder a un miembro de $\mathcal{F}^{\prime}$ que contiene 
a $a$, por lo tanto $a \in V(\mathbf{X})$. De la misma manera lo probamos para $b$ y $c$.

Finalmente probaremos que $\mathbf{X}$ es un subgrafo de $\mathbf{G}-T$. Sea $e$ una arista de $\mathbf{X}$, debemos ver que $e$ no está en ningún clique que contiene a $u_{\ell}$ ni a $v$. Por definición de $\mathbf{X}$, e es la arista de un clique de $\mathbf{G}$ tal que corresponde a un vértice de $\mathbf{S}^{\prime}$. Por la afirmación 3.1.2 los vértices extremos de $e$ no son $u_{\ell}$ ni $v$, entonces $e$ no está en un clique que contenga $u_{\ell}$, pues estos cliques son unicamente $A, B$ y $C$. Por la misma razón, si e está en un clique que contiene a $v$, este clique debe corresponder a un vértice de $\mathbf{S}$, pero entonces resulta que hay un vértice de $\mathbf{S}^{\prime}$ adyacente a un vértice de $\mathbf{S}$, lo cual contradice la planaridad de $\mathbf{H}_{\ell-1}$.

En la demostración anterior no solo hemos probado que existe un grafo planar en la imagen inversa sino que lo hemos construido, probamos entonces el siguiente corolario.

Corolario 3.1.12. Sea $\mathbf{G}$ un grafo clique $\mathbf{K}_{4}$-libre, Dom el conjunto de vértices dominados de $\mathbf{G}$ y $\mathcal{F}$ la familia $\mathcal{C}(\mathbf{G}) \bigcup(\{u\})_{u \in \text { Dom. }} . \mathbf{G} \in K($ Planares $)$ si y solo si $L(\mathcal{F})$ es planar.

\section{Complejidad Algorítmica}

Cada una de las condiciones fijadas en el Teorema 3.1.11 de esta sección, es testeable en tiempo polinomial para un grafo $\mathbf{K}_{4}$-libre, por lo tanto $K$ (Planares) $\cap \mathbf{K}_{\mathbf{4}}-$ libre es una clase de grafos con reconocimiento polinomial.

\subsection{2 $K($ Planares $) \cap \mathbf{K}_{\mathbf{3}}-$ libre}

Buscamos caracterizar $K($ Planares $) \cap \mathbf{K}_{\mathbf{3}}-$ libre.

El grafo línea de un grafo dado $\mathbf{G}$ se define como el grafo intersección de la familia de conjuntos $\mathcal{E}_{\mathbf{G}}=(e)_{e \in E(\mathbf{G})}$, cuyos miembros son las aristas del grafo. El grafo línea de $\mathbf{G}$ se denota $\operatorname{Lin}(\mathbf{G})$. De acuerdo a las definiciones dadas en el Capítulo 2 resulta que $\operatorname{Lin}(\mathbf{G})=L\left(\mathcal{E}_{\mathbf{G}}\right)$.

El siguiente Teorema debido a Sedláček caracteriza a los grafos tales que su grafo línea es un grafo planar. 
Teorema 3.1.13. [23, Sedláček] Sea $\mathbf{G}$ un $\operatorname{grafo}$. Lin $(\mathbf{G})$ es planar si y solo si

\section{1. $\mathbf{G} \in$ Planares,}

2. Todo vértice de $\mathbf{G}$ tiene grado a lo sumo 4, y

3. Si un vértice de $\mathbf{G}$ tiene grado 4 , entonces es un vértice de corte.

Si $\mathbf{G}$ es un grafo $\mathbf{K}_{3}$ - libre, sin vértices aislados, todo clique de $\mathbf{G}$ es un $\mathbf{K}_{2}$, es decir hay una correspondencia biunívoca entre las aristas y los cliques de $\mathbf{G}$, de donde $\mathcal{C}(\mathbf{G})=\mathcal{E}_{\mathbf{G}}$ y por lo tanto

$$
\mathbf{G} \text { es } \mathbf{K}_{3}-\text { libre } \Longrightarrow K(\mathbf{G})=\operatorname{Lin}(\mathbf{G})
$$

Es claro entonces que el Teorema de Sedlác̆ek nos permite deducir cuando un grafo $\mathbf{K}_{3}$ - libre está en $K^{-1}$ (Planares), pero no nos sirve para ver cuando un grafo $\mathbf{K}_{3}$ - libre está en $K$ (Planares), pues aunque el grafo en cuestión sea $\mathbf{K}_{3}$-libre, los grafos en la imagen inversa por el operador $K$ pueden no ser $\mathbf{K}_{3}-$ libre.

Sin embargo a través del Teorema 3.1.11 podemos reducir un problema en el otro: todo grafo $\mathbf{K}_{3}$ - libre satisface inmediatamente el hecho de ser grafo clique, y satisface la segunda y la tercer hipótesis del Teorema 3.1.11, se deduce que un grafo $\mathbf{K}_{3}$-libre pertenece a $K(\mathbf{P l a n a r e s})$ si y solo si $K(\mathbf{G}) \in$ Planares, i.e. si y solo si $\mathbf{G}$ pertenece a $K^{-1}$ (Planares); por lo tanto hemos probado el siguiente teorema.

Teorema 3.1.14. Sea $\mathbf{G}$ un grafo $\mathbf{K}_{3}$ - libre. Son equivalentes:

1. $\mathbf{G} \in K($ Planares $)$.

2. $\mathbf{G} \in K^{-1}$ (Planares).

3. (a) $\mathbf{G} \in$ Planares,

(b) Todo vértice de $\mathbf{G}$ tiene grado a lo sumo 4, y

(c) Si un vértice de $\mathbf{G}$ tiene grado 4 , entonces es un vértice de corte. 
El siguiente corolario nos dice que un grafo $\mathbf{K}_{3}$ - libre que no es planar, no "viene" por el operador clique de un grafo planar y no "va" por el operador clique a un grafo planar.

Corolario 3.1.15. Si $\mathbf{G}$ es $\mathbf{K}_{3}$-libre $y \mathbf{G} \notin$ Planares, entonces $\mathbf{G} \notin K(\mathbf{P l a n a r e s})$ y $K(\mathbf{G}) \notin$ Planares.

\section{Complejidad Algorítmica}

Cada una de las condiciones fijadas en el el punto 3 del Teorema 3.1.14 de esta sección, son testeables en tiempo polinomial para un grafo $\mathbf{K}_{3}-$ libre, por lo tanto $K$ (Planares) $\cap \mathbf{K}_{\mathbf{3}}$ - libre y $K^{-1}$ (Planares $) \cap \mathbf{K}_{\mathbf{3}}$ - libre son clases de grafos con reconocimiento polinomial.

\subsubsection{Ejemplos}

Probaremos facilmente a partir de los resultados anteriores que ninguno de los grafos de la Figura 3.5 está en $K$ (Planares), es decir en la imagen inversa por el operador $K$ de estos grafos, no hay ningún grafo planar.

$\mathbf{G}_{1}$ es $\mathbf{K}_{3}$ - libre y tiene un vértices de grado 4 que no es de corte, por lo tanto $\mathbf{G}_{1} \notin K($ Planares).

$\mathbf{G}_{2}$ es $\mathbf{K}_{4}$-libre y $K\left(\mathbf{G}_{2}\right)$ no es planar, concluimos que $\mathbf{G}_{2} \notin K$ (Planares).

$K\left(\mathbf{G}_{3}\right)$ es planar pero $\mathbf{G}_{3}$ tiene una arista que pertenece a cuatro cliques, entonces $\mathbf{G}_{3} \notin K($ Planares $)$.

$K\left(\mathbf{G}_{4}\right)$ es planar, toda arista de $\mathbf{G}_{4}$ está en a lo sumo tres cliques pero hay una arista que está en exactamente tres cliques y los vértices $a, b$ and $c$ no satisfacen la tercer hipótesis del Teorema 3.1.11, por lo tanto concluimos que $\mathbf{G}_{4} \notin K$ (Planares). 

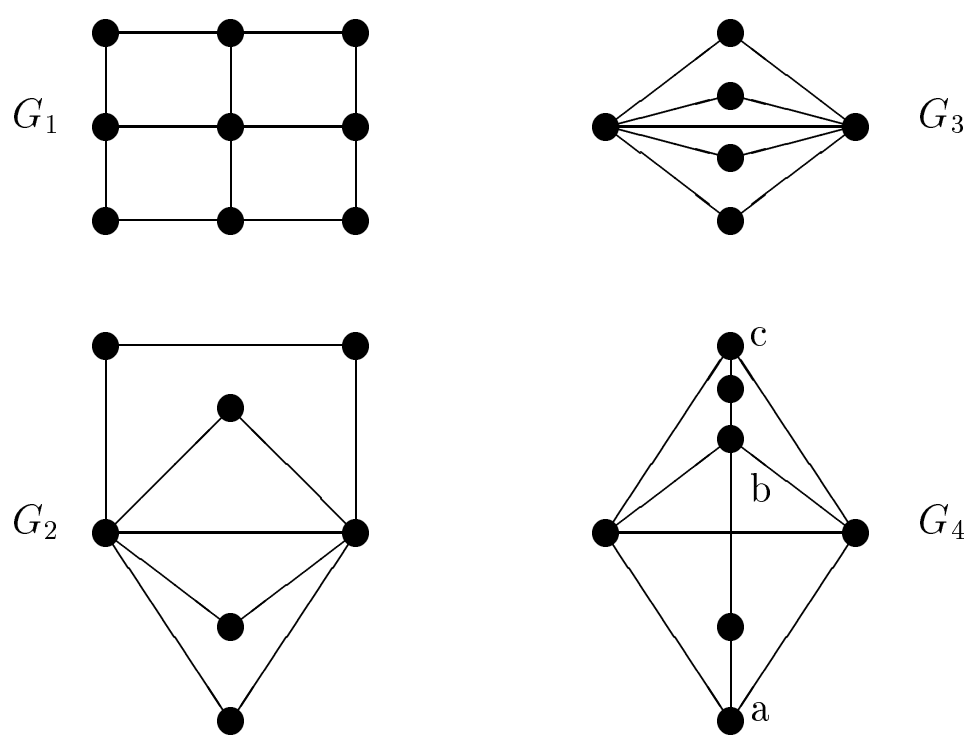

Figura 3.5: Grafos no pertenecientes a $K$ (Planares).

Como $\mathbf{K}_{3,3}$ es un ejemplo de un grafo en las condiciones del Corolario 3.1.15, ha quedado probado que

$$
\mathbf{K}_{3,3} \notin K(\text { Planares }) \text {. }
$$

\section{$3.2 \quad K^{-1}$ (Planares)}

Probaremos en esta sección que la clase de grafos $K^{-1}$ (Planares) tiene reconocimiento polinomial. Esto se deduce facilmente del hecho que estos grafos tiene una cantidad de cliques que depende linealmente de la cantidad de vértices, y del hecho de que los grafos planares son reconocibles eficientemente.

Lema 3.2.1. Si $\mathbf{H} \in K^{-1}$ (Planares), entonces en $\mathcal{C}(\mathbf{H})$, hay a lo sumo 4 cliques mutuamente intersectantes. 
Corolario 3.2.2. Si $\mathbf{H} \in K^{-1}$ (Planares), y $v \in V(\mathbf{H})$ entonces $v$ pertenece a lo sumo a cuatro cliques de $H$.

Corolario 3.2.3. Si $\mathbf{H} \in K^{-1}$ (Planares), la cantidad de cliques de $\mathbf{H}$ es menor o igual a cuatro veces la catidad de vértices de $\mathbf{H}$.

Teorema 3.2.4. La clase de grafos $K^{-1}$ (Planares) tiene reconocimiento polinomial.

Demostración: Dado un grafo $\mathbf{H}$, comenzamos a listar sus cliques. Por ejemplo en [17] se da un algoritmo que trabaja en tiempo $\mathbf{O}(n . m)$ por cada clique listado. $\mathrm{Si}$ la cantidad de cliques listados supera cuatro veces la cantidad de vértices, 4.n, nos detenemos pues el grafo dado no está en $K^{-1}$ (Planares). Sino, hemos obenido $\mathcal{C}(\mathbf{H})$ en tiempo $\mathbf{O}\left(n^{2} . m\right)$. Computar $K(\mathbf{H})$ a partir de la lista de los cliques es del orden de $k^{2}$ donde $k$ es la cantidad de cliques, por lo tanto, en este caso, resulta polinomial en $n$. Finalmente se chequea si $K(\mathbf{H})$ es o no un grafo planar, para esto en [17] hay referencias de distintos algoritmos que trabajan en tiempo lineal.

Con posterioridad a la obtención de este resultado vimos que una versión similar, para la clase $K^{-1}\left(\mathbf{K}_{\mathbf{k}}-\right.$ libre $)$ en general y para $K^{-1}$ (Planares) en particular, figura en [21].

Observemos que como

$$
\text { Planares } \subseteq \mathrm{K}_{\mathbf{5}}-\text { libre, }
$$

entonces

$$
K^{-1}(\text { Planares }) \subseteq K^{-1}\left(\mathbf{K}_{\mathbf{5}}-\text { libre }\right) .
$$

La inclusión es estricta pues por ejemplo el mismo grafo $\mathbf{H}$, ilustrado en la Figura 3.1 , pertenece a $K^{-1}\left(\mathbf{K}_{5}\right.$-libre) (pues $K(\mathbf{H})=\mathbf{K}_{3,3}$ y $\mathbf{K}_{3,3}$ es $\mathbf{K}_{5}$-libre) pero $\mathbf{H}$ no pertenece a $K^{-1}$ (Planares) (pues $K(\mathbf{H})=\mathbf{K}_{3,3}$ y $\mathbf{K}_{3,3}$ no es planar).

El siguiente resultado, nos dice que pertenecer a $K^{-1}$ (Planares) es una propiedad hereditaria para subgrafos inducidos. 
Lema 3.2.5. Si $\mathbf{H} \in K^{-1}$ (Planares), $y \mathbf{H}^{\prime} \subseteq \mathbf{H}$ entonces $\mathbf{H}^{\prime} \in K^{-1}$ (Planares).

Demostración: Si $\mathbf{H}^{\prime} \subseteq \mathbf{H}$, por el segundo item de la Proposición 2.4.1, $K\left(\mathbf{H}^{\prime}\right) \leq$ $K(\mathbf{H})$. Como $K(\mathbf{H})$ es planar, por ser la planaridad hereditaria, resulta $K\left(\mathbf{H}^{\prime}\right)$ planar.

Como todo bloque es subgrafo inducido, resulta el siguiente corolario referido a los bloques de los grafos en $K^{-1}$ (Planares),.

Corolario 3.2.6. Si $\mathbf{G} \in K^{-1}$ (Planares) entonces todo bloque de $\mathbf{G}$ pertenece a $K^{-1}$ (Planares).

La recíproca de este corolario no es en general cierta. El mismo grafo $\mathbf{K}_{1,5}$ nos proporciona un contraejemplo. 


\section{Capítulo 4}

\section{Grafos Planares Helly y 3-Helly}

En este capítulo damos una caracterización de los grafos planares Helly y de los 3Helly, mediante una sencilla descripción de los triángulos extendidos de estos grafos. Mostramos de esta forma la potencialidad del uso de los triángulos extendidos en la caracterización de otras clases de grafos, más allá de la clase de los grafos Helly. En el próximo capítulo volveremos sobre el tema de los triángulos extendidos.

\subsection{Introducción}

Hemos visto que un grafo se dice Helly si la familia de cliques del grafo tiene la propiedad Helly; más generalmente, dado $k \in \mathbf{N}$, diremos que un grafo es $k-H e l l y$, si la familia de cliques del grafo tiene la propiedad $k-$ Helly. Llamaremos $\mathbf{k}-$ Helly a la clase de todos los grafos $k-$ Helly. Es trivial que:

$$
\begin{gathered}
\text { Helly } \subseteq \ldots \subseteq \mathbf{k}+\mathbf{1}-\text { Helly } \subseteq \mathbf{k}-\text { Helly } \subseteq \ldots \subseteq \mathbf{3}-\text { Helly } \\
\mathbf{2}-\text { Helly }=\mathbf{1}-\text { Helly }=\text { Grafos }
\end{gathered}
$$

Roberts y Spencer demuestran en [22] que si $w(\mathbf{G}) \leq k$ y $\mathcal{F}$ es una familia de completos de $\mathbf{G}$ entonces $\mathcal{F}$ tiene la propiedad Helly si y solo si $\mathcal{F}$ tiene la propiedad k-Helly. 
Se deduce que si $w(\mathbf{G}) \leq k$ entonces,

$$
\mathbf{G} \in \text { Helly } \Longleftrightarrow \mathbf{G} \in \mathbf{k}-\text { Helly }
$$

por lo tanto un grafo planar es grafo Helly si y solo si es $4-$ Helly.

Resultan las siguientes relaciones,

$$
\begin{gathered}
\text { Planares } \cap \text { Helly }=\text { Planares } \cap 4-\text { Helly, } \\
\text { Planares } \cap \text { Helly } \subseteq \text { Planares } \cap 3-\text { Helly } \subseteq \text { Planares. }
\end{gathered}
$$

Si bien no se conoce una caracterización de los grafos $k-$ Helly en general, los grafos Helly tienen reconocimiento polinomial a traves del algoritmo que surge de la caracterización dada por Jayme Swarcfiter, basada en características de los triángulos extendidos del grafo.

El triángulo extendido de un grafo $\mathbf{G}$, relativo al triángulo $T \in T(\mathbf{G})$, es el subgrafo inducido en $\mathbf{G}$ por los vértices adyacentes al menos a dos vértices de $T$. En adelante notaremos $\mathbf{T}^{\prime}$, al triángulo extendido de $\mathbf{G}$ relativo $T$.

Teorema 4.1.1. [25, Szwarcfiter] G es un grafo Helly si y solo si todo triángulo extendido de $\mathbf{G}$ tiene un vértice universal.

A continuación daremos una caracterización particular de los grafos planares Helly y una caracterización de los grafos planares $3-$ Helly, basadas en propiedades de los triángulos extendidos del grafo.

\subsection{Las caracterizaciones}

Dado un grafo $\mathbf{G}$ y un triángulo $T=\{x, y, z\} \in T(\mathbf{G})$, llamamos:

$$
\begin{gathered}
V_{x y}=\{v \in V(\mathbf{G})-T / v \sim x, v \sim y, v \nsim z\}, \\
V_{x z}=\{v \in V(\mathbf{G})-T / v \sim x, v \nsim y, v \sim z\}, \\
V_{y z}=\{v \in V(\mathbf{G})-T / v \nsim x, v \sim y, v \sim z\}, \\
V_{x y z}=\{v \in V(\mathbf{G}) / v \sim x, v \sim y, v \sim z\} .
\end{gathered}
$$


Es claro que $V\left(\mathbf{T}^{\prime}\right)$ es unión disjunta de $V_{x y}, V_{x z}, V_{x y}$ y $V_{x y z}$.

Decimos que:

$\mathbf{T}^{\prime}$ es de tipo 1 si alguno de los conjuntos $V_{x y}, V_{x z}$ o $V_{y z}$ es vacío.

$\mathbf{T}^{\prime}$ es de tipo 2 si existen vértices $x_{1}, y_{1}, z_{1}$ y $u$ de $\mathbf{G}$ tal que

$V_{x y}=\left\{z_{1}\right\}, V_{x z}=\left\{y_{1}\right\}, V_{y z}=\left\{x_{1}\right\}, V_{x y z}=\{u\}, x_{1} \sim u, y_{1} \sim u, \mathrm{y} z_{1} \sim u$.

$\mathbf{T}^{\prime}$ es de tipo 3 si existen vértices $x_{1}, y_{1}, z_{1}, u$ y $u^{\prime}$ de $\mathbf{G}$ tal que

$V_{x y}=\left\{z_{1}\right\}, V_{x z}=\left\{y_{1}\right\}, V_{y z}=\left\{x_{1}\right\}, V_{x y z}=\left\{u, u^{\prime}\right\}, x_{1} \sim u, y_{1} \sim u, \mathrm{y} z_{1} \sim u$.

Obsérvese que un triángulo extendido de tipo 2, de un grafo planar, es isomorfo al grafo $\mathbf{T}_{2}$ de la Figura 4.1 y que un triángulo extendido de tipo 3 de un grafo planar es isomorfo al grafo $\mathbf{T}_{3}$ de la misma figura, en otras palabras estas clases de grafos contienen un único grafo planar. Se debe a que los grafos $\mathbf{T}_{2}$ y $\mathbf{T}_{3}$ son planares maximales: por el agregado de cualquier otra arista entre dos de sus vértices se obtiene un grafo que no es planar. Además, se deduce de esto que si $\mathbf{T}_{1} \circ \mathbf{T}_{2}$ son subgrafos de un grafo planar $\mathbf{G}$ entonces, son subgrafos inducidos de $\mathbf{G}$.

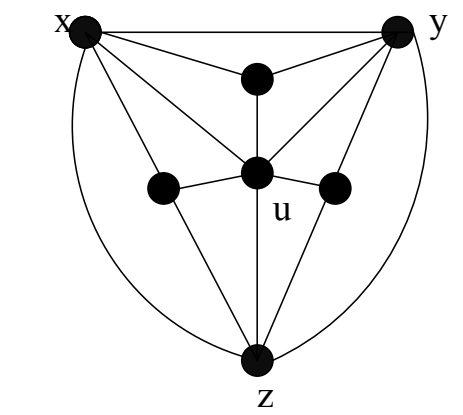

Grafo $\mathbf{T}_{2}$

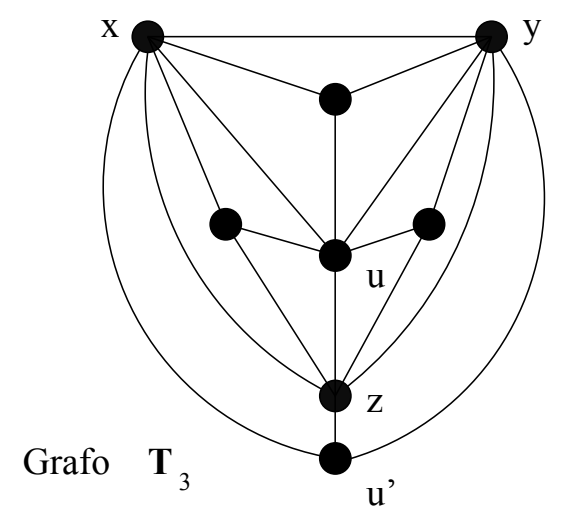

Figura 4.1: Los grafos $\mathbf{T}_{2}$ y $\mathbf{T}_{3}$.

Teorema 4.2.1. Sea $\mathbf{G}$ un grafo planar. $\mathbf{G}$ es Helly si y solo si para todo triángulo $T$ de $\mathbf{G}$ se verifica que $\mathbf{T}^{\prime}$ es de tipo 1 o de tipo 2. 
Demostración: Sea G Helly, y supongamos que existe un triángulo $T=\{x, y, z\}$ tal que $\mathbf{T}^{\prime}$ no es de tipo 1 , entonces $V_{x y}, V_{x z}$ y $V_{y z}$ son todos no vacíos, de donde si llamamos $u$ al vértice universal de $\mathbf{T}^{\prime}$ (que sabemos existe pues $\mathbf{G}$ es Helly), resulta que $u$ no es $x, y$ ni $z$. Por otra parte, por ser $u$ universal, $u$ es adyacente a $x$, a $y$ y a $z$ y a cada uno de los vértices pertenecientes a $V_{x y}, V_{x z} \mathrm{y} V_{y z}$. Así tenemos que el grafo $\mathbf{T}_{2}$ es un subgrafo de $\mathbf{T}^{\prime}$, y además es inducido pues es planar maximal. Si en $\mathbf{T}^{\prime}$ hubiere algún otro vértice además de los de $\mathbf{T}_{2}$, este vértice, llamémosle $h$, debería ser adyacente al menos a dos vértices de $T$ y a $u$, pero esto contradice la planaridad de $\mathbf{T}^{\prime}$ como se ve en la siguiente figura.

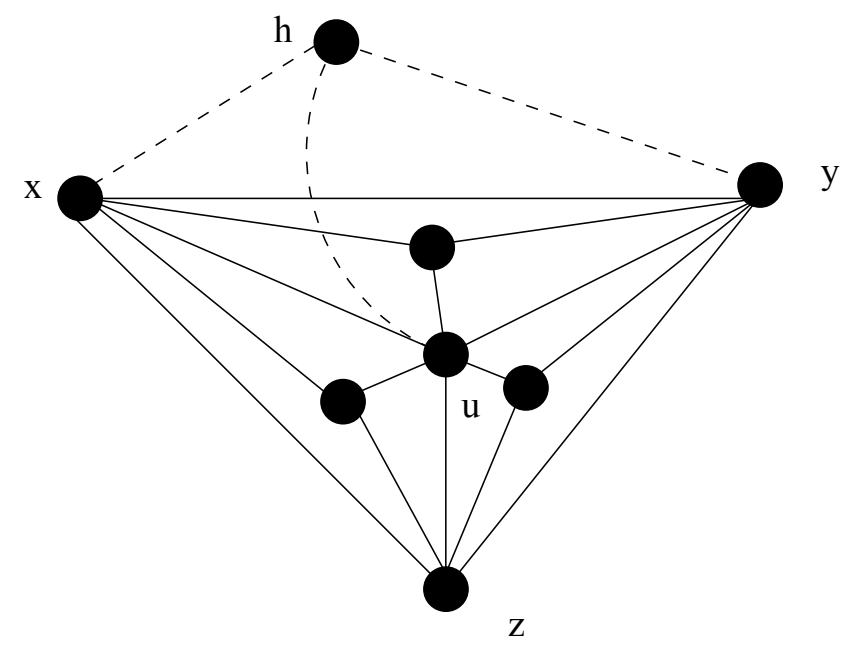

Concluimos que $\mathbf{T}^{\prime}$ debe ser el grafo $\mathbf{T}_{2}$. La recíproca es inmediata por cuanto todo triángulo extendido del tipo 1 o 2 tiene un vértice universal.

Teorema 4.2.2. Sea $\mathbf{G}$ un grafo planar. $\mathbf{G}$ es 3 - Helly si y solo si para todo triángulo $T$ de $\mathbf{G}$ se verifica que $\mathbf{T}^{\prime}$ es de tipo 1, 2 o 3.

Demostración: Sea $\mathbf{G}$ un grafo planar $3-$ Helly y sea $T=\{x, y, z\} \in T(\mathbf{G})$ tal que $\mathbf{T}^{\prime}$ no es de tipo 1 , ni 2 . Por no ser de tipo 1 existen vértices $x_{1} \in V_{y z}, y_{1} \in V_{x z} \mathrm{y}$ $z_{1} \in V_{x y}$. Como los cliques que contienen los completos $\left\{x_{1}, y, z\right\},\left\{x, y_{1}, z\right\}$ y $\left\{x, y, z_{1}\right\}$ 
son distintos entre si y son mutuamente intersectante, deben tener un vértice en común, pues $\mathbf{G}$ es $3-$ Helly. Llamemos $u$ a este vértice; es claro que $u$ no es ninguno de los vértices $x, y, z, x_{1}, y_{1}$ ni $z_{1}$. Resulta entonces que el grafo $\mathbf{T}_{2}$ es subgrafo (inducido) de $\mathbf{T}^{\prime}$. Como $\mathbf{T}^{\prime}$ no es del tipo $2, \mathbf{T}^{\prime}$ no puede ser el grafo $\mathbf{T}_{2}$, por lo tanto $\mathbf{T}^{\prime}$ debe tener algún otro vértice. Llamemos $u^{\prime}$ a este otro vértice de $\mathbf{T}^{\prime}$. Supongamos sin pérdida de generalidad que $u^{\prime}$ es adyacente a $x$ y a $y$, como se ve en la siguiente figura.

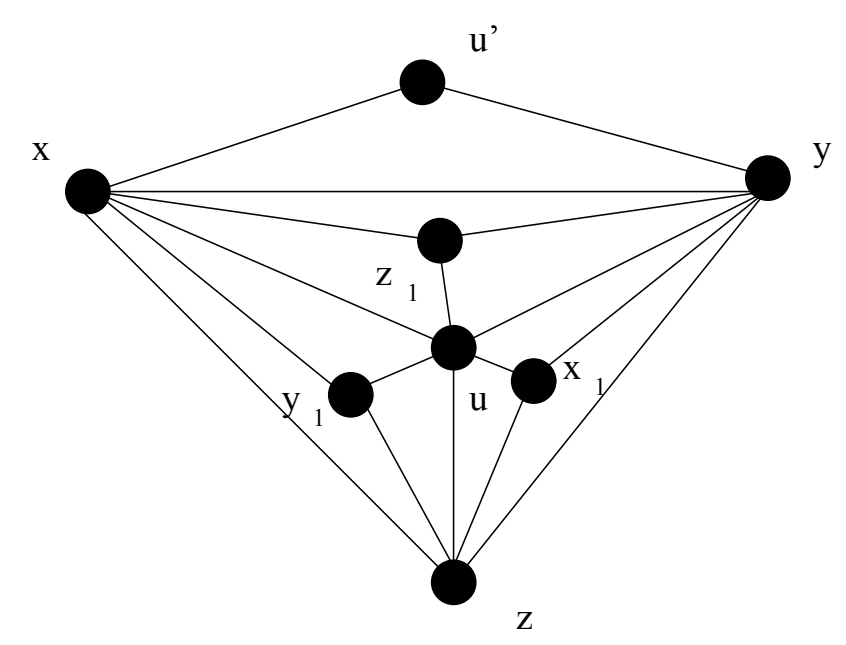

Ahora, el completo $\left\{x, y, u^{\prime}\right\}$ está contenido en un clique que no es ninguno de los ya nombrados, y este clique es mutuamente intersectante con los cliques $\left\{x, y_{1}, z, u\right\} \mathrm{y}$ $\left\{x_{1}, y, z, u\right\}$, por lo tanto los tres debe tener un vértice en común. Como los cliques contienen a lo sumo cuatro vérices, el vértice común debe ser $z$ o $u$. Si fuera $u$, resultaría $u \sim u^{\prime}$ de donde $\mathbf{T}^{\prime}$ no sería planar.

Concluimos, entonces, que $z$ debe ser el vértice común, por lo tanto $z \sim u^{\prime}$ y así tenemos que el grafo $\mathbf{T}_{3}$ es subgrafo inducido de $\mathbf{T}^{\prime}$. Ahora, para ver que $\mathbf{T}^{\prime}$ es el grafo $\mathbf{T}_{3}$, basta ver que en $\mathbf{T}^{\prime}$ no hay más vértices que los ya descriptos. Supongamos que $\mathbf{T}^{\prime}$ tiene otro vértice $w$, este debe ser adyacente al menos a dos vértices de $T$, repitiendo el razonamiento anterior resulta que $w$ también debe ser adyacente al tercer vértice de $T$, con lo cual $\mathbf{T}^{\prime}$ no resulta planar, contradicción.

Recíprocamente, sea $\mathbf{G}$ un grafo tal que para todo triángulo el correspondiente 
triángulo extendido es de tipo 1, 2 o 3. Supongamos que G no es 3-Helly, entonces existen cliques de $\mathbf{G}, C_{1}, C_{2}$, y $C_{3}$, mutuamente intersectante y sin un vértice en común. Es fácil ver que deben existir vértices $x, y, z, x_{1}, y_{1}, z_{1}$ tal que: $C_{1} \supset\left\{x_{1}, y, z\right\}, x \notin C_{1}$; $C_{2} \supset\left\{x, y_{1}, z\right\}, y \notin C_{2} ;$ y $C_{3} \supset\left\{x, y, z_{1}\right\}, z \notin C_{3}$, como se ilustra en la siguiente figura.

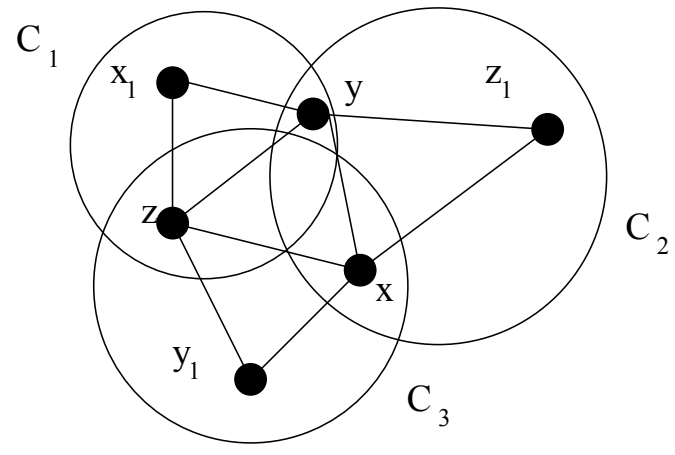

Llamemos $T$ al triángulo de vértices $x, y, z$. Es claro que $\mathbf{T}^{\prime}$ no es de tipo 1 , por lo tanto $\mathbf{T}^{\prime}$ debe ser $\mathbf{T}_{2}$ o $\mathbf{T}_{3}$, pero, en cualquiera de los dos casos, el vértice $u$ descripto en la definición de $\mathbf{T}_{2}$ y $\mathbf{T}_{3}$ pertenecería a $C_{1}, C_{2}$ y $C_{3}$, lo cual contradice que estos cliques no tiene un vértice en común.

Problema Abierto 4.2.1. Hacer un análisis similar al que hacemos en esta sección, para un grafo cualquiera, no necesariamente planar. Es decir, caracterizar los grafos que son $k-$ Helly en base a propiedades de sus triángulos extendidos. 


\section{Capítulo 5}

\section{$K($ Grafos $) \cap$ Planares}

En este capítulo generalizamos la noción de triángulo extendido y obtenemos un marco común para los resultados de Szwarcfiter (Teorema 4.1.1) y de Roberts-Spencer (Teorema 2.4.1).

Damos una condición necesaria para que un grafo planar sea grafo clique (que todo triángulo extendido del grafo lo sea) y caracterizamos totalmente los triángulos extendidos de un grafo planar que son grafos clique. Dejamos abierta una posibilidad de caracterización total de los grafos clique planares mediante la compatibilidad de los cubrimientos de sus triángulos extendidos.

Sin pérdida de generalidad, en vista del Lema 2.4.4, en adelante consideraremos unicamente grafos tales que sus cliques contienen al menos tres vértices.

\section{$5.1 \quad$ Introducción}

Sabemos que un grafo es grafo clique si existe una familia de completos del grafo tal que: i) cubre las aristas y ii) tiene la propiedad Helly. Todo grafo tiene una particular familia de completos que satisface i): la familia de sus cliques. Cuando esta familia satisface ii) el grafo se dice grafo Helly y es claro entonces que Helly $\subseteq K$ (Grafos). Desde este punto de vista la relación entre la idea de grafo Helly y la de grafo clique es bien clara. Sin embargo, si pensamos en la caracterización de los grafos Helly 
que permite el reconocimiento eficiente de esta clase, se pierde la idea intuitiva de la relación entre dichas clases de grafos. En otras palabras, nos preguntamos como se relacionan las siguientes definiciones:

Grafo Helly = todo triángulo extendido del grafo tiene un vértice universal.

Grafo Clique = existe una familia de completos del grafo que cubre las aristas y tiene la propiedad Helly.

Veremos que ambas se relacionan en torno a los triángulos del grafo: en la primer definición el vértice universal es un vértice común a todos los cliques que continen al menos dos vértices del triángulo; la segunda definición es equivalente a pedir que exista un vértice común a todos los completos de la familia que contienen al menos dos vértices del triángulo. En la Sección 5.2 desarrollamos formalmente estas ideas. Hemos encontrado en esta forma de ver a los grafos clique - trabajando en torno a sus triángulos - una poderosa herramienta.

Resulta además del análisis realizado que un grafo es grafo Helly si y solo si lo es cada uno de sus triángulos extendidos. Basándonos en esta idea, encontramos que si un grafo planar es grafo clique entonces lo es cada uno de sus triángulos extendidos. Además caracterizamos los triángulos extendidos de un grafo planar que son grafos clique.

El análisis y entendimiento de este caso particular de los grafos planares nos ha permitido desarrollar una nueva caracterización de los grafos clique en general, la cual es motivo del próximo capítulo.

\subsection{Triángulo extendido según una familia de com- pletos}

Sea $\mathbf{G}$ un grafo y $V_{1}$ y $V_{2}$ dos subconjuntos de vértices de $\mathbf{G}$. Diremos que $V_{1}$ y $V_{2}$ están pegados si su intersección contiene al menos dos vértices, es decir si

$$
\left|V_{1} \cap V_{2}\right| \geq 2
$$


Sea $\mathcal{F}=\left(F_{i}\right)_{i \in I}$ una familia de completos de $\mathbf{G}$ y $T$ un triángulo de $\mathbf{G}$. Notamos $\mathcal{F}_{T}$ a la subfamilia de $\mathcal{F}$ formada por los miembros pegados a $T$, en caso en que estos existan, es decir

$$
\mathcal{F}_{T}=\left(F_{i}\right)_{i \in I^{\prime}} \quad \text { si } \quad I^{\prime}=\left\{i \in I /\left|F_{i} \cap T\right| \geq 2\right\} \neq \emptyset .
$$

En adelante toda referencia a $\mathcal{F}_{T}$ queda supeditada a su existencia; por ejemplo si decimos "para todo $T \in T(\mathbf{G}), \mathcal{F}_{T} \ldots$. ", nos referimos a todo $T \in T(\mathbf{G})$ para el cual existe $\mathcal{F}_{T}$, mientras que nada se dice sobre los triángulos $T$ para los cuales no existe $\mathcal{F}_{T}$.

Por otra parte cabe destacar que esta salvedad no es necesaria si $\mathcal{F}$ cubre las aristas de $\mathbf{G}$, pues en este caso para todo triángulo tiene sentido hablar de $\mathcal{F}_{T}$.

El triángulo extendido de $\mathbf{G}$ según la familia $\mathcal{F}$, relativo al triángulo $T$, que denotaremos $\mathbf{T}_{\mathcal{F}}$, es el subgrafo de $\mathbf{G}$ inducido por los vértices que son elementos de $\mathcal{F}_{T}$, es decir

$$
\mathbf{T}_{\mathcal{F}}=\mathrm{G}\left[\cup \mathcal{F}_{T}\right]
$$

Si $\mathcal{F}=\mathcal{C}(G)$ llamamos a $\mathbf{T}_{\mathcal{F}}$ simplemente triángulo extendido de $\mathbf{G}$ relativo a $T$ y lo notamos $\mathbf{T}^{\prime}$. Es fácil probar que esta definición de triángulo extendido coincide con la dada por Szwarcfiter, es decir $\mathbf{T}^{\prime}$ es el subgrafo de $\mathbf{G}$ inducido por todos los vértices de $\mathbf{G}$ adyacentes al menos a dos vértices de $T$; por lo tanto esta noción de triángulo extendido según una familia $\mathcal{F}$ de conjuntos de vértices generaliza la de triángulo extendido, ya conocida.

Llamamos en general triángulo extendido, a cualquier grafo $\mathbf{G}$ tal que alguno de sus triángulos $T$, satisface $\mathbf{T}^{\prime}=\mathbf{G}$, y en caso de no ser necesario omitiremos especificar cuál es triángulo $T$ de $\mathbf{G}$ en estas condiciones.

Lema 5.2.1. Sean $\mathcal{F}$ y $\mathcal{M}$ familias de completos de un grafo $\mathbf{G}$ y $T$ un triángulo cualquiera de $\mathbf{G}$,

$$
\mathcal{F} \preceq \mathcal{M} \Longrightarrow \mathcal{F}_{T} \preceq \mathcal{M}_{T} \quad \text { y } \quad \mathbf{T}_{\mathcal{F}} \subseteq \mathbf{T}_{\mathcal{M}}
$$


Demostración: Si $F$ es un miembro de $\mathcal{F}_{T}$, entonces es miembro de $\mathcal{F}$. Como $\mathcal{F} \preceq \mathcal{M}$, existe $M$ miembro de $\mathcal{M}$ tal que $F \subseteq M$. Por otra parte como $F$ contiene al menos dos vértices de $T$, entonces $M$ tiene al menos dos vértices de $T$, de donde $M$ es un miembro de $\mathcal{M}_{T}$. Hemos probado que $\mathcal{F}_{T} \preceq \mathcal{M}_{T}$.

Para demostrar que $\mathbf{T}_{\mathcal{F}} \subseteq \mathbf{T}_{\mathcal{M}}$ basta ver que todo vértice del primero es un vértice del segundo pues se trata de subgrafos inducidos. Como $\mathcal{F}_{T} \preceq \mathcal{M}_{T}$, resulta la siguiente inclusión

$$
V\left(\mathbf{T}_{\mathcal{F}}\right)=\cup \mathcal{F}_{T} \subseteq \cup \mathcal{M}_{T}=V\left(\mathbf{T}_{\mathcal{M}}\right)
$$

Lema 5.2.2. Sea $\mathcal{F}$ una familia de completos de un grafo $\mathbf{G}$. $\mathcal{F}$ tiene la propiedad Helly si y solo si para todo triángulo $T \in T(\mathbf{G}), \mathcal{F}_{T}$ tiene la propiedad de Helly; si y solo si para todo triángulo $T \in T(\mathbf{G}), \mathcal{F}_{T}$ tiene intersección total no vacía.

Demostración: Sea $T$ un triángulo de $\mathbf{G}$. Como $\mathcal{F}_{T}$ es subfamilia de $\mathcal{F}$, si $\mathcal{F}$ tiene la propiedad Helly entonces $\mathcal{F}_{T}$ tienen la propiedad Helly, de donde $\cap \mathcal{F}_{T} \neq \emptyset$, pues es mutuamente intersectante. Recíprocamente, sea $\mathcal{F}$ una familia de completos de un grafo $\mathbf{G}$, tal que para todo triángulo $T \in T(\mathbf{G}), \cap \mathcal{F}_{T} \neq \emptyset$. De acuerdo a la Proposición 2.1.1 para ver que la familia $\mathcal{F}$ tiene la propiedad Helly basta ver que dados tres elementos cualesquiera de $\cup \mathcal{F}: x, y$, z; la subfamilia de $\mathcal{F}$, que notaremos $\mathcal{F}^{\prime}$, formada por aquellos miembros que contienen al menos dos de estos tres vértices, tiene intersección total no vacía. Lo vemos: si $T=\{x, y, z\} \in T(\mathbf{G})$, es claro que $\mathcal{F}^{\prime}=\mathcal{F}_{T}$, por lo tanto $\cap \mathcal{F}^{\prime}=\cap \mathcal{F}_{T} \neq \emptyset$ por hipótesis. Si $\{x, y, z\} \notin T(\mathbf{G})$ es por que al menos dos de los vértices no son adyacentes entre sí, supongamos $x$ e $y$, entonces como los miembros de $\mathcal{F}$ son completos de $\mathbf{G}$, se deduce fácilmente que todos los miembros de $\mathcal{F}^{\prime}$ contienen a $z$, por lo tanto $\cap \mathcal{F}^{\prime} \neq \emptyset$. 
Lema 5.2.3. Sea $\mathcal{F}$ una familia de completos de un grafo $\mathbf{G}$ y $T \in T(\mathbf{G})$. Si $\mathcal{F}_{T}$ tiene intersección total no vacía entonces $\mathbf{T}_{\mathcal{F}}$ tiene un vértice universal. La recíproca vale en el caso en que $\mathcal{F}=\mathcal{C}(\mathbf{G})$.

Demostración: Sea $u \in \cap \mathcal{F}_{T}$. Como los miembros de $\mathcal{F}_{T}$ son completos de $\mathrm{G}, u$ es adyacente a todos los vértices de $\cup \mathcal{F}_{T}$, como a su vez $V\left(\mathbf{T}_{\mathcal{F}}\right)=\cup \mathcal{F}_{T}$, resulta que $u$ es un vértice universal de $\mathbf{T}_{\mathcal{F}}$.

Recíprocamente, sea $u$ un vértice universal de $\mathbf{T}_{\mathcal{C}(\mathbf{G})}$, queremos probar que $u \in$ $\cap \mathcal{C}(\mathbf{G})_{T}$. Sea $C \in \mathcal{C}(\mathbf{G})_{T}$, como $V\left(\mathbf{T}_{\mathcal{C}(\mathbf{G})}\right)=\cup\left(\mathcal{C}(\mathbf{G})_{T}\right) \supseteq C$, entonces $u$ es adyacente a todos los vértices de $C$. Como $C$ es un clique de $\mathbf{G}$, resulta $u \in C$.

El siguiente lema muestra la relación existente entre los cliques de un grafo dado y los cliques de uno cualquiera sus triángulos extendidos.

Lema 5.2.4. Sea $\mathbf{G}$ un grafo y $\mathbf{T}^{\prime}$ el triángulo extendido de $\mathbf{G}$ relativo al triángulo T. Valen las siguientes proposiciones

- $\mathcal{C}\left(\mathbf{T}^{\prime}\right) \prec \mathcal{C}(\mathbf{G})$, pero en general $\mathcal{C}\left(\mathbf{T}^{\prime}\right) \nsubseteq \mathcal{C}(\mathbf{G})$.

- $\mathcal{C}\left(\mathbf{T}^{\prime}\right)_{T}=\mathcal{C}(\mathbf{G})_{T}$, y por lo tanto $\mathcal{C}(\mathbf{G})_{T} \subseteq \mathcal{C}\left(\mathbf{T}^{\prime}\right)$, pero en general $\mathcal{C}(\mathbf{G})_{T} \neq \mathcal{C}\left(\mathbf{T}^{\prime}\right)$.

Demostración: Como $\mathbf{T}^{\prime}$ es un subgrafo inducido de $\mathbf{G}$, es claro que todo clique de $\mathbf{T}^{\prime}$ es un completo de $\mathbf{G}$, por lo tanto para probar la primer afirmación basta ver que si $C_{1}$ y $C_{2}$ son cliques distintos de $\mathbf{T}^{\prime}$, no pueden estar contenidos en un mismo clique $C$ de $\mathbf{G}$. Si fuera así resultaría que $C_{1} \cup C_{2} \subseteq C$, por lo tanto $C_{1} \cup C_{2}$ sería un completo de $\mathbf{G}$ y por lo tanto de $\mathbf{T}^{\prime}$, lo cual es una contradicción.

Ahora demostremos la segunda afirmación: Sea $C$ perteneciente a $\mathcal{C}(\mathbf{G})_{T}$, entonces $C$ es un clique de $\mathbf{G}$ pegado al triángulo $T$; como por definición $\mathbf{T}^{\prime}=\mathbf{G}\left[\cup \mathcal{C}(\mathbf{G})_{T}\right]$, resulta que $C$ es clique de $\mathbf{T}^{\prime}$; como además $C$ está pegado a $T$ resulta que $C \in \mathcal{C}\left(\mathbf{T}^{\prime}\right)_{T}$. Hemos probado que $\mathcal{C}(\mathbf{G})_{T} \subseteq \mathcal{C}\left(\mathbf{T}^{\prime}\right)_{T}$. Por otra parte como $\mathcal{C}\left(\mathbf{T}^{\prime}\right) \prec \mathcal{C}(\mathbf{G})$ entonces $\mathcal{C}\left(\mathbf{T}^{\prime}\right)_{T} \prec \mathcal{C}(\mathbf{G})_{T}$. De ambos resultados surge $\mathcal{C}(\mathbf{G})_{T}=\mathcal{C}\left(\mathbf{T}^{\prime}\right)_{T}$. 
En el grafo $\mathbf{G}$ de la siguiente Figura, consideremos el triángulo $T=\{x, y, z\}$. Claramente $w \notin V\left(\mathbf{T}^{\prime}\right) . \quad C_{1}=\{u, v, z\}$ es clique de $\mathbf{T}^{\prime}$ pero no de $\mathbf{G}$, por lo tanto $\mathcal{C}\left(\mathbf{T}^{\prime}\right) \not \mathcal{C}(\mathbf{G})$. Además $C_{1} \notin \mathcal{C}(\mathbf{G})_{T}$, simplemente por no ser clique de $\mathbf{G}$, por lo tanto $\mathcal{C}(\mathbf{G})_{T} \neq \mathcal{C}\left(\mathbf{T}^{\prime}\right)$.

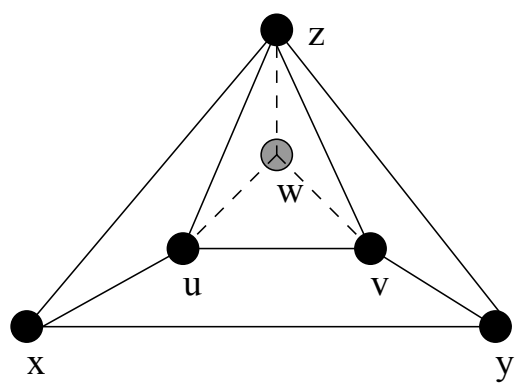

Grafo G

El siguiente Teorema generaliza el dado por Szwarcfiter como caracterización de los grafos Helly.

Teorema 5.2.5. Sea $\mathbf{G}$ un grafo. Las siguientes proposiciones son equivalentes:

- $\mathbf{G}$ es un grafo Helly.

- $\mathcal{C}(\mathbf{G})$ tiene la propiedad Helly.

- $T \in T(\mathbf{G}) \Longrightarrow \mathcal{C}(\mathbf{G})_{T}$ tiene la propiedad Helly.

- $T \in T(\mathbf{G}) \Longrightarrow \mathcal{C}(\mathbf{G})_{T}$ tiene intersección total no vacía.

- $T \in T(\mathbf{G}) \Longrightarrow \mathbf{T}^{\prime}$ tiene un vértice universal.

- $T \in T(\mathbf{G}) \Longrightarrow \mathbf{T}^{\prime}$ es un grafo Helly.

Demostración: $\mathbf{G}$ es un grafo Helly si y solo si (definición) $\mathcal{C}(\mathbf{G})$ tiene la propiedad Helly; si y solo si (Lema 5.2.2) para todo triángulo $T \in T(\mathbf{G}), \mathcal{C}(\mathbf{G})_{T}$ tiene la propiedad Helly; si y solo si (Lema 5.2.2) para todo triángulo $T \in T(\mathbf{G}), \cap \mathcal{C}(\mathbf{G})_{T} \neq \emptyset$, 
si y solo si (Lema 5.2.3) para todo triángulo $T \in T(\mathbf{G}), \mathbf{T}_{\mathcal{C}(\mathbf{G})}=\mathbf{T}^{\prime}$ tiene un vértice universal, entonces para todo triángulo $T \in T(\mathbf{G}), \mathbf{T}_{\mathcal{C}(\mathbf{G})}=\mathbf{T}^{\prime}$ es un grafo Helly. Falta demostrar que si $\mathbf{G}$ es tal que todo triángulo extendido es Helly entonces $\mathbf{G}$ es un grafo Helly, para esto basta ver que para todo $T \in T(\mathbf{G}), \cap \mathcal{C}(\mathbf{G})_{T} \neq \emptyset$. Sea $T \in T(\mathbf{G})$, por ser $\mathbf{T}^{\prime}$ Helly resulta que $\cap \mathcal{C}\left(\mathbf{T}^{\prime}\right)_{T} \neq \emptyset$; por el Lema 5.2.4 $\mathcal{C}(\mathbf{G})_{T} \subseteq \mathcal{C}\left(\mathbf{T}^{\prime}\right)_{T}$, con lo cual $\cap \mathcal{C}(\mathbf{G})_{T} \neq \emptyset$.

Análogamente se demuestra el siguiente Teorema que generaliza el de Roberts y Spencer.

Teorema 5.2.6. Sea $\mathbf{G}$ un grafo. Las siguientes proposiciones son equivalentes:

- $\mathbf{G}$ es un grafo clique.

- Existe $\mathcal{F}$ un cubrimiento por completos de $\mathbf{G}$ tal que, $\mathcal{F}$ tiene la propiedad Helly.

- Existe $\mathcal{F}$ un cubrimiento por completos de $\mathbf{G}$ tal que, $T \in T(\mathbf{G}) \Longrightarrow \mathcal{F}_{T}$ tiene la propiedad Helly.

- Existe $\mathcal{F}$ un cubrimiento por completos de $\mathbf{G}$ tal que, $T \in T(\mathbf{G}) \Longrightarrow \mathcal{F}_{T}$ tiene intersección total no vacía.

- Existe $\mathcal{F}$ un cubrimiento por completos de $\mathbf{G}$ tal que, $T \in T(\mathbf{G}) \Longrightarrow \mathbf{T}_{\mathcal{F}}$ tiene un vértice universal y este vértice universal está en todo miembro de $\mathcal{F}_{T}$.

- Existe $\mathcal{F}$ familia $\mathcal{R}-\mathcal{S}$ de $\mathbf{G}$ tal que, $T \in T(\mathbf{G}) \Longrightarrow \mathcal{F}_{T}$ es familia $\mathcal{R}-\mathcal{S}$ de $\mathbf{T}_{\mathcal{F}}$

Cualquiera de las proposiciones precedentes implica que

- Existe $\mathcal{F}$ un cubrimiento por completos de $\mathbf{G}$ tal que, $T \in T(\mathbf{G}) \Longrightarrow \mathbf{T}_{\mathcal{F}}$ es un grafo clique.

Podríamos preguntarnos si vale también la equivalencia de la última proposición del Teorema anterior. Es decir, si existe una familia de completos que cubre las aristas 
de $\mathbf{G}$, tal que todo triángulo extendido de acuerdo a esta familia es un grafo clique entonces $\mathbf{G}$ es grafo clique?

La respuesta es no. Como contraejemplo tenemos el sencillo caso del grafo Pirámide, Figura 2.2, con su cubrimiento por completos formado por las aristas, que llamaremos $\mathcal{F}$. Es claro que para todo $T \in T(\mathbf{P}), \mathbf{T}_{\mathcal{F}}=\mathbf{T}$ y por lo tanto es grafo clique, sinembargo sabemos que $\mathbf{P}$ no lo es.

Ocurre que $\mathbf{T}_{\mathcal{F}}$ tiene un vértice universal, pero este vértice no esta en todos los miembros de $\mathcal{F}_{T}$, como se ilustra en la siguiente figura.

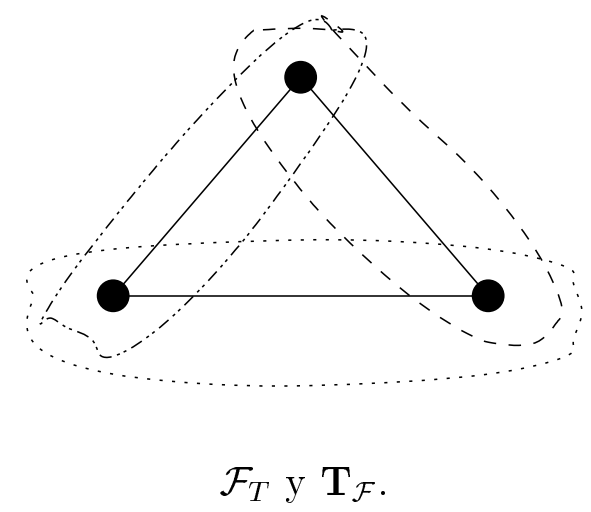

Equivalentemente se puede pensar que $\mathbf{T}_{\mathcal{F}}$ es grafo clique, pero la familia $\mathcal{R}-\mathcal{S}$ que admite no es jutamente $\mathcal{F}_{T}$. Esta idea es la de compatibilidad de cubrimientos que desarrollamos al final de la sección siguiente.

\subsection{Condición necesaria para grafo clique planar}

Observemos que la última equivalencia del Teorema 5.2.6 dice que a partir de una familia $\mathcal{R}-\mathcal{S}, \mathcal{F}$, de un grafo dado se puede obtener una familia $\mathcal{R}-\mathcal{S}$ de ciertos subgrafos inducidos en torno a cada triángulo del grafo: $\mathbf{T}_{\mathcal{F}} ;$ y viceversa. Podríamos querer usar esta idea para tatar de construir una familia $\mathcal{R}-\mathcal{S}$ del grafo dado empezando por construir una ramilia $\mathcal{R}-\mathcal{S}$ de las estructuras más sencillas $\mathbf{T}_{\mathcal{F}}$; pero el inconveniente, claro está, es que $\mathbf{T}_{\mathcal{F}}$ depende también de la familia $\mathcal{F}$ que queremos construir. A priori lo único que sabemos es que $\mathbf{T}_{\mathcal{F}}$ es subgrafo inducido de $\mathbf{T}^{\prime}$. 
Justamente para el caso de los grafos planares probamos que es posible obtener a partir de una familia $\mathcal{R}-\mathcal{S}$ del grafo, una familia $\mathcal{R}-\mathcal{S}$ de cada triángulo extendido.

En lo que sigue utilizaremos la siguiente notación: si $F$ es un conjunto de vértices, $F \supseteq\{x, y, \ldots, \not u, \not \nu\}$, significa que $x, y, \ldots$ pertenecen a $F$, mientras que $u, v, \ldots$, no pertenecen a $F$.

Si $\mathcal{F}=\left(F_{i}\right)_{i \in I}$ una familia $\mathcal{R}-\mathcal{S}$ y $\mathbf{T}^{\prime}$ un triángulo extendido, $\mathcal{F} \cap \mathbf{T}^{\prime}$ denota la familia de completos de $\mathbf{T}^{\prime},\left(F_{i}^{\prime}\right)_{i \in I^{\prime}}$, donde

$$
I^{\prime}=\left\{i \in I /\left|F_{i} \cap V\left(\mathbf{T}^{\prime}\right)\right| \geq 3\right\} \quad \text { y } \quad F_{i}^{\prime}=F_{i} \cap V\left(\mathbf{T}^{\prime}\right) .
$$

Teorema 5.3.1. Sea $\mathbf{T}^{\prime}$ un triángulo extendido de un grafo planar $\mathbf{G}$. Si $\mathcal{F}$ es una familia $\mathcal{R}-\mathcal{S}$ de $\mathbf{G}$ entonces $\mathcal{F} \cap \mathbf{T}^{\prime}$ es una familia $\mathcal{R}-\mathcal{S}$ de $\mathbf{T}^{\prime}$.

Demostración: Sea $\mathcal{F}=\left(F_{i}\right)_{i \in I}$ y $T=\{x, y, z\}$. Llamemos $\mathcal{F}^{\prime}$ a $\mathcal{F} \cap \mathbf{T}^{\prime}$. Es claro que los miembros de $\mathcal{F}^{\prime}$ son completos de $\mathbf{T}^{\prime}$. Veamos que cubren las aristas de $\mathbf{T}^{\prime}$. Si no fuera así existiría una aristas $u v$ de $\mathbf{T}^{\prime}$ que no está en un completo de $\mathcal{F}^{\prime}$, esto significa, por definición de $\mathcal{F}^{\prime}$ y porque $\mathcal{F}$ cubre las aristas de $\mathbf{G}$, que en todo completo de $\mathcal{F}$ conteniendo a $u$ y a $v$ no hay otro vértice de $\mathbf{T}^{\prime}$ :

$$
F \in \mathcal{F}, u \in F, v \in F \Longrightarrow F \cap V\left(\mathbf{T}^{\prime}\right)=\{u, v\}
$$

Veamos que esto es un absurdo:

Caso 1: $u, v \in T$, podemos suponer sin pérdida de generalidad $u=x, v=y$. Por la implicación 5.3.1 el triángulo $\{\mathrm{u}, \mathrm{v}, \mathrm{z}\}$ no es un completo de $\mathcal{F}$, de donde deben existir miembros de $\mathcal{F}: F_{1} \supset\{u, v, \not k\}, F_{2} \supset\{v, z, \not u\}, F_{3} \supset\{u, z, \not p\}$. Por ser estos completos mutuamente intersectantes, tienen un vértice en común, que claramente no es ninguno de los ya nombrados, sea $w$. Resulta que $w$ es un vértice de $\mathbf{T}^{\prime}$ y está en el completo $F_{1}$, miembro de $\mathcal{F}$ con $u$ y $v$, lo cual contradice la implicación 5.3.1. Caso 2: $u \in T$ y $v \notin T$, sin pérdida de generalidad podemos suponer $u=x$ y $v$ adyacente a $y$ y repetir el razonamiento anterior para el triángulo $\{u, v, y\}$. 


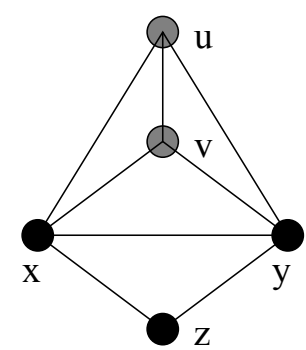

a

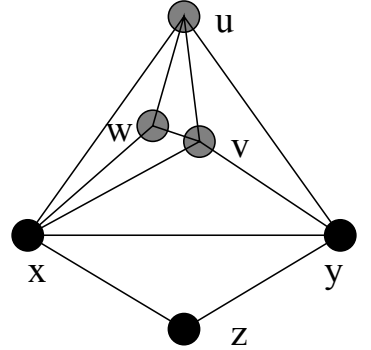

b

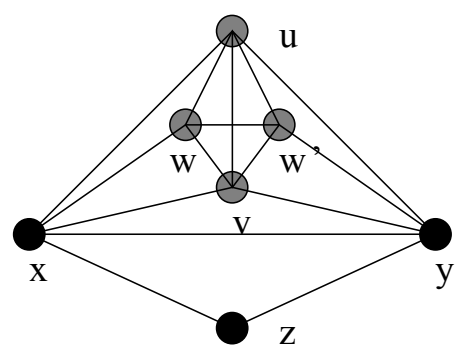

c

Figura 5.1:

Caso 3: $u \notin T, v \notin T$.

Subcaso 3.1: $u$ y $v$ son adyacentes a un mismo par de vértices de $T$, podemos suponer a $x$ y a $y$ (Fig.5.1.a). Entonces, por la implicación 5.3.1 el triángulo $\{\mathrm{u}, \mathrm{v}, \mathrm{x}\}$ no es un miembro de $\mathcal{F}$, por lo tanto deben existir en $\mathcal{F}: F_{1} \supset\{u, v, \not x\}, F_{2} \supset\{x, u, \not p\}$, $F_{3} \supset\{x, v, \not \mu\}$.Por ser mutuamente intersectantes, tienen un vértice en común que llamaremos $w$. Nuevamente por la implicación 5.3.1, $w$ no puede ser un vértice de $\mathbf{T}^{\prime}$, por lo tanto no es ninguno de los hasta ahora indicados (Fig.5.1.b), además $w \not y, w \not z$. Tenemos así: $F_{1} \supset\{u, v, w, \not x, \not y, \not z\}, F_{2} \supset\{x, u, w, \not v, \not y, \not z\}$, $F_{3} \supset\{x, v, w, \not h, \not y, \not z\}$. Por otra parte, existirán miembros de $\mathcal{F}$ cubriendo las aristas vy y uy: $F_{4} \supset\{v, y, \not u, \not \omega\}, F_{5} \supset\{u, y, \not \nu, \not \omega\}$, claramente no son los anteriores. Por ser mutuamente intersectantes $F_{1}, F_{4}$ y $F_{5}$ deben tener un vértice en común; este vértice no puede ser $x, y, z, u, v$, ni $w$. Se trata entonces de un vértice $w^{\prime}$ (Fig.5.1.c). Así resulta: $F_{1} \supset\left\{u, v, w, w^{\prime}\right\}, F_{2} \supset\{x, u, w\}, F_{3} \supset\{x, v, w\}, F_{4} \supset\left\{v, y, w^{\prime}\right\}$, $F_{5} \supset\left\{u, y, w^{\prime}\right\}$, pero en estas condiciones hay una subdivisión de $\mathbf{K}_{5}$ con lo cual $\mathbf{T}^{\prime}$ no es planar. Contradicción.

Subcaso 3.2: $u$ y $v$ no son adyacentes a un mismo par de vértices de $T$. Podemos suponer $u$ es adyacente a $x$ y a $y$ mientras que $v$ es adyacente a $y$ y a $z$ (Fig.5.2.a). Razonando como antes deben existir miembros de $\mathcal{F}: F_{1} \supset\{u, v, w, \not k, \not y, \not k\}, F_{2} \supset$ $\{u, y, w, \not x, \not \notin, \not p\}, F_{3} \supset\{v, y, w, \not x, \not 火, \not \mu\}$, con $w \notin V\left(\mathbf{T}^{\prime}\right)$ (Fig.5.2.b). Ahora, a su vez, 


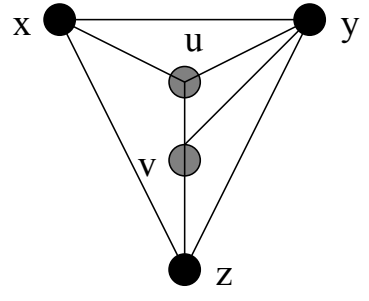

a

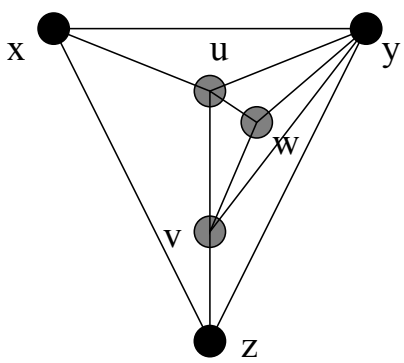

b

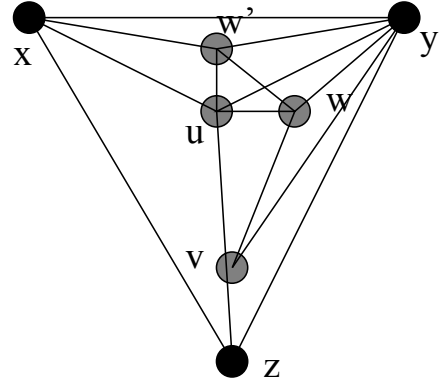

C

Figura 5.2:

hay dos posibilidades:

Subcaso 3.2.1.: $u, x, y$ están en un mismo miembro de $\mathcal{F}$. Este completo resulta mutuamente intersectante con $F_{1}$ y $F_{3}$, por lo tanto tienen un vértice común que como en los casos anteriores debe pertenecer a $V\left(\mathbf{T}^{\prime}\right)$, lo cual es absurdo, por la implicación 5.3.1.

Subcaso 3.2.2: Si $u, x, y$ no están en un mismo miembro de $\mathcal{F}$, entonces en $\mathcal{F}$ además de los completos nombrados al comienzo del subcaso 3.2, hay completos satisfaciendo: $F_{4} \supset\{x, y, \not k, \not u, \not \omega\}, F_{5} \supset\{x, u, \not y, \not \nu, \not \omega\}$, que deben tener un vértice en común con $F_{2}$. Este vértice en común no puede ser $x, y, z, v, u$, ni $w$, entonces existe un vértice $w^{\prime}$ (Fig.5.2.c) y completos $F_{1} \supset\{u, v, w\}, F_{2} \supset\left\{u, y, w, w^{\prime}\right\}, F_{3} \supset\{v, y, w\}$, $F_{4} \supset\left\{x, y, w^{\prime}\right\}, F_{5} \supset\left\{x, u, w^{\prime}\right\}$, por lo tanto existe una subdivisión de $\mathbf{K}_{5}$, de donde $\mathbf{T}^{\prime}$ no es planar. Contradicción.

Con esto hemos probado que la familia de completos $\mathcal{F}^{\prime}$ cubre las aristas de $\mathbf{T}^{\prime}$. Resta probar, entonces, que $\mathcal{F}^{\prime}$ es una familia con la propiedad Helly.

Supongamos existe una subfamilia $\mathcal{F}^{\prime \prime}=\left(F_{i}^{\prime}\right)_{i \in I^{\prime \prime}}, I^{\prime \prime} \subseteq I^{\prime}$, mutuamente intersectante sin un vértice en común y minimal en el sentido que cualquier subfamilia de $\mathcal{F}$ " tiene intersección total no vacía, entonces para todo $i \in I^{\prime}$ existe un vértice $v_{i}$ de $\mathbf{G}$ tal que $v_{i}$ pertenece a todo miembro de $\mathcal{F}$ " salvo a $F_{i}^{\prime}$. 
Es claro que si $i \neq j$, entonces $v_{i} \neq v_{j}$, y que

$$
\left\{v_{i}, i \in I^{\prime \prime}\right\} \text { es un completo de } \mathbf{G} \text {. }
$$

Por otra parte, por como fue definida $\mathcal{F}^{\prime}$, resulta que

$$
\bigcap_{i \in I^{\prime \prime}} F_{i}^{\prime}=\left\{\bigcap_{i \in I^{\prime \prime}} F_{i}\right\} \cap V\left(\mathbf{T}^{\prime}\right)=\emptyset
$$

Pero, como $\mathcal{F}$ tiene la propiedad Helly, sabemos que existe un vértice $u$ tal que $u \in \bigcap_{i \in I}$ " $F_{i}$, entonces se deduce de la ecuación anterior que $u \notin V\left(\mathbf{T}^{\prime}\right)$.

Ahora, como los completos $F_{i}^{\prime}$ contiene al menos 3 vértices y los completos $F_{i}$ contiene a lo sumo 4 vértices, resulta que

$$
i \in I^{\prime \prime} \Longrightarrow F_{i}=F_{i}^{\prime} \cup\{u\}
$$

De los enunciados 5.3.2 y 5.3.3, resulta que si $|I "| \geq 4$, en $\mathbf{G}$ hay un completo con 5 vértices lo cual contradice la planaridad de G. Si $\left|I^{\prime \prime}\right|=3$ y los vértices $v_{i}, i \in I$ ", con el vértice $u$ no conforman un $\mathbf{K}_{5}$, la situación es la descripta en la Figura 5.3.

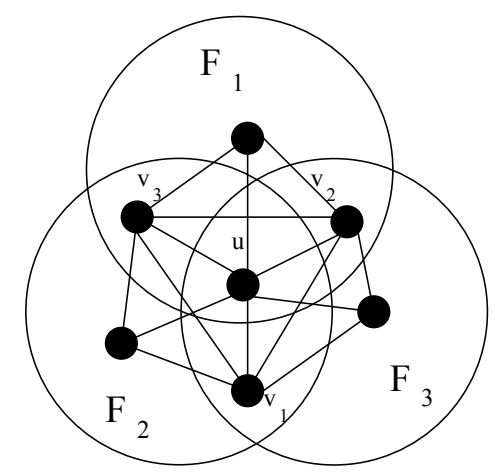

Figura 5.3: $F_{i}^{\prime}=F_{i}-\{u\} . u \notin V\left(\mathbf{T}^{\prime}\right)$. 
Entre los vértices distintos de $u$, del subgrafo de $\mathbf{G}$ descripto en dicha figura, ocurre que: i) no hay dos pertenecientes al triángulo $T$, pues el vértice $u$ no pertenece a $V\left(\mathbf{T}^{\prime}\right)$ y ii) cada uno de ellos es adyacentes al menos a dos vértices de $T$, pues cada uno de ellos está en $V\left(\mathbf{T}^{\prime}\right)$.

Es fácil probar a partir de i) y ii) que en $\mathbf{G}$ hay una subdivisión de $\mathbf{K}_{5}$, lo cual es nuevamente una contradicción.

Hemos probado entonces que no existe una subfamilia mutuamente intersectante con intersección total vacía.

Corolario 5.3.2. Sea $\mathbf{G}$ un grafo planar. Si $\mathbf{G}$ es un grafo clique, entonces todo triángulo extendido de $\mathbf{G}$ es también un grafo clique.

La recíproca de este resultado no es cierta como se puede ver en el siguiente ejemplo.

\section{Compatibilidad de cubrimientos}

Consideremos el grafo planar $\mathbf{G}$ de la siguiente figura. Mostramos una representación no plana para resaltar la simetría del grafo.

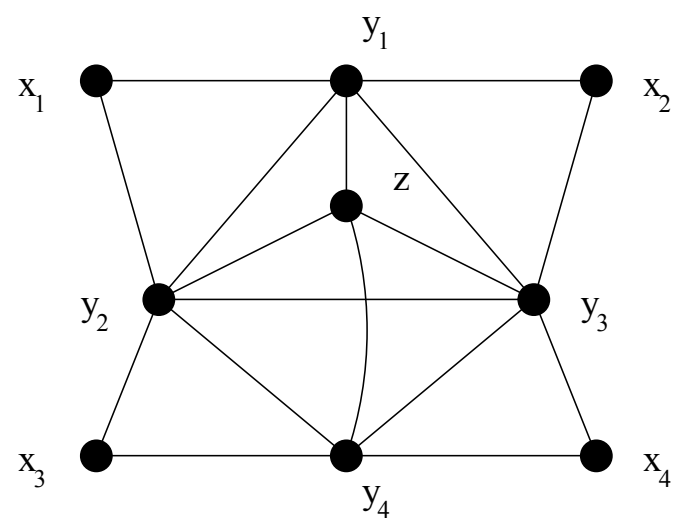

Grafo G

El grafo tiene cuatro clases de triángulos: 
i)Los cuatro triángulos de las esquinas: $\left\{x_{1}, y_{1}, y_{2}\right\},\left\{x_{2}, y_{1}, y_{3}\right\},\left\{x_{3}, y_{2}, y_{4}\right\}$, y $\left\{x_{4}, y_{3}, y_{4}\right\}$. En los cuatro casos el correspondiente triángulo extendido de $\mathbf{G}$ es el de la siguiente figura, que claramente es grafo clique.

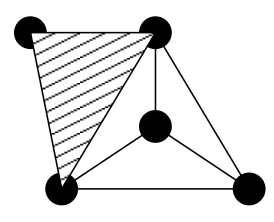

ii)Los cuatro centrales: $\left\{z, y_{1}, y_{2}\right\},\left\{z, y_{1}, y_{3}\right\},\left\{z, y_{2}, y_{4}\right\}$ y $\left\{z, y_{3}, y_{4}\right\}$. En los cuatro casos el correspondiente triángulo extendido de $\mathbf{G}$ es el de la siguiente figura, que claramente es grafo clique.

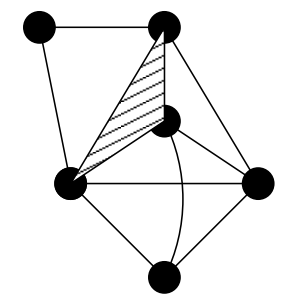

iii)El triángulo $\left\{z, y_{2}, y_{3}\right\}$. El correspondiente triángulo extendido, que se muestra a continuación, es grafo clique.

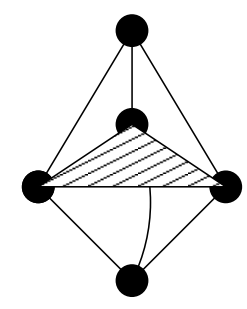

Finalmente, iv) los triángulos $T_{1}=\left\{y_{1}, y_{2}, y_{3}\right\}$ y $T_{2}=\left\{y_{2}, y_{3}, y_{4}\right\}$. La Figura 5.3 muestra el grafo que llamaremos $\mathbf{A}$, isomorfo a $\mathbf{T}_{1}^{\prime}$ y a $\mathbf{T}_{2}^{\prime}$, el cual también es grafo clique. 


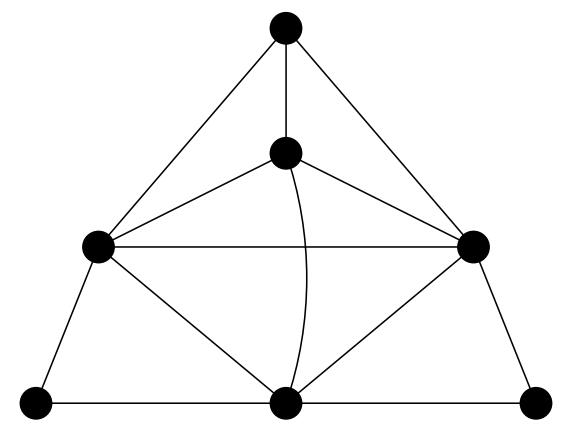

Figura 5.4: Grafo A.

Sin embargo, a pesar de que todos los triángulos extendidos son grafo clique, el grafo G no lo es, con lo cual probamos que no vale la recíproca del Corolario 5.3.2. Pero esto, como es de esperar, no contradice el Teorema 5.3.1. Es más, se puede probar que $\mathbf{G}$ no es grafo clique a partir de dicho teorema, veamos:

I)El grafo A admite una única familia $\mathcal{R}-\mathcal{S}$, a saber:

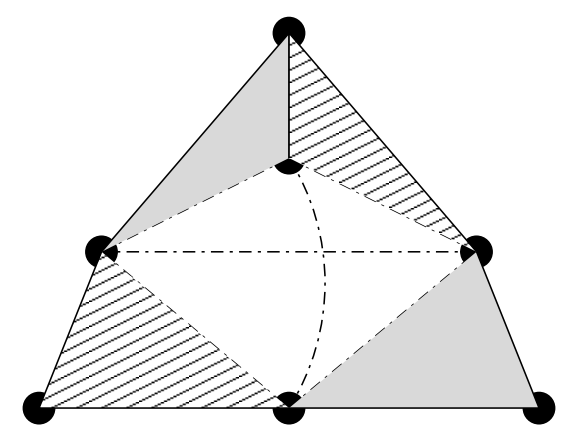

Familia $\mathcal{R}-\mathcal{S}$ de $\mathbf{A}$.

II)Se deduce que $\mathbf{T}_{1}^{\prime}$ admite una única familia $\mathcal{R}-\mathcal{S}$,

$$
\left\{z, y_{2}, y_{4}\right\},\left\{z, y_{3}, y_{4}\right\},\left\{z, y_{1}, y_{2}, y_{3}\right\},\left\{x_{1}, y_{1}, y_{2}\right\},\left\{x_{2}, y_{1}, y_{3}\right\}
$$

III)Igualmente $\mathbf{T}_{2}^{\prime}$ admite una única familia $\mathcal{R}-\mathcal{S}$, 


$$
\left\{z, y_{1}, y_{2}\right\},\left\{z, y_{1}, y_{3}\right\},\left\{z, y_{2}, y_{3}, y_{4}\right\},\left\{x_{3}, y_{2}, y_{4}\right\},\left\{x_{4}, y_{3}, y_{4}\right\}
$$

Ahora, supongamos exite $\mathcal{F}$ familia $\mathcal{R}-\mathcal{S}$ de $\mathbf{G}$.

Por el Teorema 5.3.1, $\mathcal{F} \cap \mathbf{T}_{1}^{\prime}$ es una familia $\mathcal{R}-\mathcal{S}$ de $\mathbf{T}_{1}^{\prime}$, por lo tanto $\mathcal{F} \cap \mathbf{T}_{1}^{\prime}$ debe ser la indicada en II). De esto se deduce que $\left\{z, y_{1}, y_{2}, y_{3}\right\}$ es un miembro de $\mathcal{F}$.

Análogamente $\mathcal{F} \cap \mathbf{T}_{2}^{\prime}$ es una familia $\mathcal{R}-\mathcal{S}$ de $\mathbf{T}_{2}^{\prime}$; como vimos que $\left\{z, y_{1}, y_{2}, y_{3}\right\}$ es miembro de $\mathcal{F}$, y todos son vértices de $\mathbf{T}_{2}^{\prime}$, resulta que este debe ser un miembro de $\mathcal{F} \cap \mathbf{T}_{2}^{\prime}$, lo cual contradice III).

Concluimos a partir del Teorema que $\mathbf{G}$ no es grafo clique.

Toda esta explicación, en un lenguaje más sencillo, se reduce a decir que si un grafo planar es grafo clique, entonces cada uno de sus triángulos extendido $\mathbf{T}_{i}$ admite una familia $\mathcal{R}-\mathcal{S}, \mathcal{F}_{i}$, pero además todas estas familias deben ser compatibles en el sentido que:

$$
\forall i, j \quad \mathcal{F}_{i} \cap \mathbf{T}_{j}^{\prime} \preceq \mathcal{F}_{j}
$$

Consideramos que tras este enfoque existe una posibilidad de caracterización de los grafos clique planares y tal vez de otras clases de grafos.

\subsection{Triángulos extendidos de un Grafo Planar que son Grafos Cliques}

En la sección precedente probamos que una condición necesaria para que un grafo planar sea grafo clique, es que cada uno de sus triángulos extendidos sea grafo clique. Este resultado carecería de utilidad práctica si determinar si un triángulo extendido es grafo clique, es tan o más complejo que determinar si un grafo cualquiera es o no grafo clique; por lo tanto es de importancia saber cuando un triángulo extendido 
es grafo clique. En esta sección mostraremos una caracterización de los triángulos extendidos de un grafo planar, que son grafos clique.

A continuación presentamos el teorema de caracterización:

Teorema 5.4.1. Sea $\mathbf{G}$ un grafo planar y $T=\{x, y, z\}$ uno cualquiera de sus triángulos. $\mathbf{T}^{\prime}$ es un grafo clique si y solo si se satisface alguna de las siguientes condiciones:

a) $V_{x y}=\emptyset \circ V_{x z}=\emptyset \circ V_{y z}=\emptyset$.

b) $V_{x y}=\left\{z_{1}\right\}$ y existe $u \in V_{x y z}$ tal que $z_{1} \sim u$,o

$V_{x z}=\left\{y_{1}\right\}$ y existe $u \in V_{x y z}$ tal que $y_{1} \sim u, o$

$V_{y z}=\left\{x_{1}\right\}$ y existe $u \in V_{x y z}$ tal que $x_{1} \sim u$.

c) $V_{x y}=\left\{z_{1}, z_{1}^{\prime}\right\}, V_{x z}=\left\{y_{1}, y_{1}^{\prime}\right\}, V_{y z}=\left\{x_{1}, x_{1}^{\prime}\right\}, V_{x y z}=\{u\}, y$

$z_{1} \sim z_{1}^{\prime} \sim u, y_{1} \sim y_{1}^{\prime} \sim u, x_{1} \sim x_{1}^{\prime} \sim u$.

Obsérvese que los triángulos extendidos de un grafo planar que son de tipo 1 son exactamente los descriptos en el apartado a), mientras que los de tipo 2 y 3 están contenidos estrictamente en los descriptos en el apartado b). Además todo triángulo extendido planar que satisface c), es isomorfo al grafo B de la Figura 5.5, pues este es planar maximal.

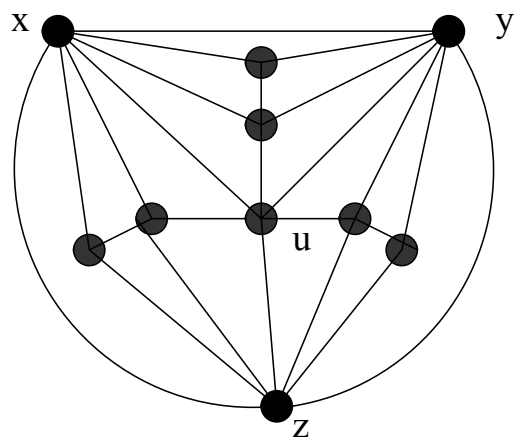

Figura 5.5: Grafo B.

Los siguientes lemas serán utilizados en la demostración del teorema precedentemente enunciado. 
Lema 5.4.2. Sea $\mathbf{G}$ un grafo planar y $T=\{x, y, z\}$ uno cualquiera de sus triángulos. Si $\mathcal{F}$ es una familia $\mathcal{R}-\mathcal{S}$ de $\mathbf{G}$ y $u_{T} \in \cap \mathcal{F}_{T}$, entonces:

1) $v \in \mathbf{T}_{\mathcal{F}}, v \neq u_{T} \Longrightarrow v \sim u_{T}$.

2) $u_{T} \in T$ o $u_{T} \in V_{x y z}$.

3) $u \in V_{x y z} \Longrightarrow u \in V\left(\mathbf{T}_{\mathcal{F}}\right)$.

4) $\left|V_{x y z}\right|=2 \Longrightarrow u_{T} \in T$.

5) $z_{1} \in V_{x y} \quad y \quad z_{1} \notin \mathbf{T}_{\mathcal{F}} \Longrightarrow V_{x y}=\left\{z_{1}\right\}$, y existe $u \in V_{x y z}$ tal que $z_{1} \sim u$,ó

$u_{T} \in V_{x y z}$ y existe $z_{1}^{\prime} \in V\left(\mathbf{T}_{\mathcal{F}}\right)$ tal que $V_{x y}=\left\{z_{1}, z_{1}^{\prime}\right\}, y z_{1} \sim z_{1}^{\prime} \sim u_{T}$.

6) $\left|V_{x y}\right|>2 \Longrightarrow u_{T} \in T$.

Demostración: 1)Es inmediato pues $V\left(\mathbf{T}_{\mathcal{F}}\right)=\cup \mathcal{F}_{T}$ y $u_{T}$ está en todos los miembros de $\mathcal{F}_{T}$.

2)Supongamos que $u_{T} \notin T$. Sea $F$ un miembro de $\mathcal{F}$ que cubre la arista $x y$ de $T$. Es claro que $F \in \mathcal{F}_{T}$, entonces $u_{T} \in F$, de donde $u_{T}$ es adyacente a $x$ y a $y$. Análogamente se prueba que es adyacente a $z$.

3)Supongamos que $u \notin V\left(\mathbf{T}_{\mathcal{F}}\right)$ (en particular $u \neq u_{T}$ ), entonces existe miembros de $\mathcal{F}$ en las siguientes condiciones: $F_{1} \supseteq\{u, x, \not y, \not z\}, F_{2} \supseteq\{u, y, \not x, \not k\}, F_{3} \supseteq\{u, z, \not x, \not y\}$. Ahora hay dos posibilidades: a)Existe un completo miembro de $\mathcal{F}$ que contiene los tres vértices de $T$, i.e. existe $F_{4} \supseteq\{x, y, z, \not \iota\}$ o b)Las aristas de $T$, no están cubiertas por un mismo miembro de $\mathcal{F}$, por lo tanto existen completos de $\mathcal{F}: F_{4} \supseteq\left\{x, y, u_{T}, \not \varnothing, \not \iota\right\}$, $F_{5} \supseteq\left\{x, z, u_{T}, \not y, \not \iota\right\}, F_{6} \supseteq\left\{y, z, u_{T}, \not x, \not \iota\right\}$, donde es claro que $u_{T} \in V_{x y z}$ y $u_{t} \neq u$. En el caso a) debe existir un vértice común a los cuatro completos y este no puede ser $x, y, z$, ni $u$, por lo que debe ser otro vértice $h$ con lo que se llega a un absurdo por planaridad. En el caso b) debe existir un vértice en común a $F_{1}, F_{2}$ y $F_{4}$, que no puede ser $x, y, z$, ni $u$, y tampoco puede ser $u_{T}$ pues, por planaridad, este no es adyacente a $u$. Aparece entonces un nuevo vértice y se tiene un absurdo por planaridad. 4)Si $u$ y $u^{\prime}$ son vértices de $V_{x y z}$, por el item anterior ambos están en $\mathbf{T}_{\mathcal{F}}$. Si uno de ellos fuera $u_{T}$, entonces el otro debería ser adyacente y esto no es posible, por lo tanto $u_{T}$ es un vértice distinto de $u$ y de $u^{\prime}$. Ahora $u \sim u_{T}$ y $u^{\prime} \sim u_{T}$, con lo cual por planaridad resulta $u_{T} \in T$. 
5)Sea $z_{1} \in V_{x y}$ y supongamos que $z_{1}$ no pertenece a ningún completo que tiene al menos dos vértices de $T$, hay entonces dos posibilidades: a) y b).

a) Existen miembros de $\mathcal{F}: F_{1} \supseteq\left\{z_{1}, x, \not y, \not k\right\}, F_{2} \supseteq\left\{z_{1}, y, \not k, \not k\right\}, F_{3} \supseteq\left\{x, y, z, \not x_{1}\right\}$.

Estos tres completos deben tener un vértice común que no es $x, y, z, z_{1}$, sea entonces $u$, es claro que $u \in V_{x y z}$.

Así tenemos $F_{1} \supseteq\left\{z_{1}, x, u, \not y, \not ̋\right\}, F_{2} \supseteq\left\{z_{1}, y, u, \not x, \not k\right\}, F_{3} \supseteq\left\{x, y, z, u, \not{ }_{1}\right\}$. Supongamos existe otro vértice $z_{1}^{\prime}$ en $V_{x y}$, hay dos posibilides:

$\left.a_{1}\right) z_{1}^{\prime} \in \mathbf{T}_{\mathcal{F}}$. En este caso existirá otro miembro de $\mathcal{F}, F_{4} \supseteq\left\{x, y, z_{1}^{\prime}, \not k, \not z_{1}, \not \mu\right\}$. Ahora $F_{1}, F_{2}$ y $F_{4}$ son mutuamente intersectantes por lo tanto deben tener un vértice en común que no es $x, y, z, z_{1}, u$, entonces debe ser un vértice $h$ distinto de los ya nombrados, pero esto contradice la planaridad.

$\left.a_{2}\right) z_{1}^{\prime} \notin \mathbf{T}_{\mathcal{F}}$. Como por planaridad $z_{1}^{\prime} \not u$, el vértice $z_{1}^{\prime}$ no está en $F_{1}, F_{2}$ ni $F_{3}$, entonces hay un miembro de $\mathcal{F}, F_{4} \supseteq\left\{z_{1}^{\prime}, x, \not y, \not 火, \not \iota\right\}, F_{5} \supseteq\left\{z_{1}^{\prime}, y, \not x, \not \subset, \not \iota\right\}$. Ahora $F_{4}$, $F_{5}$ y $F_{3}$ deben tener un vértice en común que no puede ser $x, y, z, z_{1}, u$, pero $F_{3}$ no puede contener más que cuatro vértices, con lo cual este caso tampoco es posible.

Concluimos entonces que en el caso a), en $V_{x y}$ no hay más vértices que $z_{1}$, y $z_{1} \sim u \in$ $V_{x y z}$ que es lo que queríamos probar.

b) Existen miembros de $\mathcal{F}: F_{1} \supseteq\left\{z_{1}, x, \not y, \not £\right\}, F_{2} \supseteq\left\{z_{1}, y, \not k, \not k\right\}$, y no hay un miembro de $\mathcal{F}$ donde $x, y, z$ estén juntos y por lo tanto existen completos $F_{3} \supseteq\left\{x, y, u_{T}, \not 火, \not{ }_{1}\right\}$, $F_{4} \supseteq\left\{x, z, u_{T}, \not y, \not k_{1}\right\}, F_{5} \supseteq\left\{y, z, u_{T}, \not x, \not k_{1}\right\}$. Ahora $F_{1}, F_{2}, F_{3}$ tienen un vértice común que no es $x, y, z, z_{1}$. Entonces hay dos posibilidades:

$b_{1}$ ) El vértice común es $u_{T}$. Supongamos que existe otro vértice $z_{1}^{\prime}$ en $V_{x y}$. Si $z_{1}^{\prime} \in \mathbf{T}_{\mathcal{F}}$ debe ser $z_{1}^{\prime} \sim u_{T}$ lo cual es absurdo por planaridad.

Si $z_{1}^{\prime} \notin \mathbf{T}_{\mathcal{F}}$ existe un completo cubriendo $z_{1}^{\prime} x$ y otro cubriendo $z_{1}^{\prime} y$. Como $z_{1}^{\prime} \nsim u_{T}$ no pueden ser $F_{1}, F_{2}$, ni $F_{3}$, sean $F_{6} \supseteq\left\{x, z_{1}^{\prime}, \not \mu_{T}, \not y, \not z\right\}, F_{7} \supseteq\left\{y, z_{1}^{\prime}, \not \psi_{T}, \not x, \not k\right\}$. Ahora $F_{6}$, $F_{7}$ y $F_{3}$ tienen un vértice en común que no es $x, y, z, z_{1}, u_{T}$, debe ser un $h$ distinto lo cual es absurdo por planaridad.

Conclusión del caso $b_{1}$ : en $V_{x y}$ no hay más vértices que $z_{1}$, y $z_{1} \sim u_{T} \in V_{x y z}$ que es lo que queríamos probar. 
$b_{2}$ )El vértice común no es $u_{T}$. Sea entonces un vértice $z_{1}^{\prime}$, distinto de los ya nombrados. En estas condiciones tenemos completos $F_{1} \supseteq\left\{z_{1}, x, z_{1}^{\prime}, \not y, \not k\right\}, F_{2} \supseteq\left\{z_{1}, y, z_{1}^{\prime}, \not x, \not 火\right\}$, $F_{3} \supseteq\left\{x, y, z_{1}^{\prime}, u_{T}, \not_{1}, \not z\right\}, F_{4} \supseteq\left\{x, z, u_{T}, \not y, \not z_{1}\right\}, F_{5} \supseteq\left\{y, z, u_{T}, \not x, \not z_{1}\right\}$. Con esto tenemos que $z_{1}^{\prime} \in V_{x y}, z_{1}^{\prime} \in \mathbf{T}_{\mathcal{F}}, z_{1} \sim z_{1}^{\prime} \sim u_{T} \in V_{x y z}$. Lo único que falta probar es que en $V_{x y}$ no puede haber más vértices. Supongamos que hubiere un $h$. Si $h$ fuese un vértice de $\mathbf{T}_{\mathcal{F}}, h$ debería ser adyacente a $u_{T}$ y esto es imposible por planaridad. Si no es un vértices de $\mathbf{T}_{\mathcal{F}}$, como en el caso $b_{1}$ ) debe haber otros completos con $h, x \mathrm{y}$ con $h, y$ y estos deben tener un vértice en común con $F_{3}$, que no puede ser $x, y, z_{1}^{\prime}, u_{T}$, entonces deber ser alguien distinto pero esto es imposible nuevamente por planaridad. 6) Si $\left|V_{x y}\right|>2$ por el inciso anterior todo vértice en $V_{x y}$ debe ser un vértice $\mathbf{T}_{\mathcal{F}}$, por lo tanto cada uno de ellos debe ser adyacente a $u_{T}$. Como $\mathbf{G}$ es planar se deduce $u_{T} \in T$.

Lema 5.4.3. Sea $\mathbf{T}^{\prime}$ un triángulo extendido planar relativo al triángulo $T=\{x, y, z\}$. Si $V_{x y}=\left\{z_{1}\right\}$ y $z_{1} \sim u \in V_{x y z}$, entonces:

1. $\left\{x, y, z_{1}, u\right\}$ es el único clique de $\mathbf{T}^{\prime}$ que contiene al vértice $z_{1}$.

2. Los cliques de $\mathbf{T}^{\prime}$ que contienen al vértice $u$ son $\{x, y, z, u\},\left\{x, y, z_{1}, u\right\} ;\left\{x_{1}, y, z, u\right\}$ en caso en que exista un vértice $x_{1} \in V_{y z}$ adyacente a $u ; y\left\{x, y_{1}, z, u\right\}$ en caso en que exista un vértice $y_{1} \in V_{x z}$ adyacente a $u$. Observar que u pertenece a lo sumo a cuatro cliques de $\mathbf{T}^{\prime}$.

3. Todo clique de $\mathbf{T}^{\prime}$, salvo el clique $\left\{x, y, z_{1}, u\right\}$ contiene el vértice $z$.

4. Si $V_{x y z}=\{u\}$ entonces $\{x, y, z, u\},\left\{x, y, z_{1}, u\right\}$ son los únicos cliques de $\mathbf{T}^{\prime} q u e$ contienen conjuntamente a los vértices $x$ e $y$.

Si $V_{x y z}=\left\{u, u^{\prime}\right\}$ entonces $\{x, y, z, u\},\left\{x, y, z, u^{\prime}\right\} y\left\{x, y, z_{1}, u\right\}$ son los únicos cliques de $\mathbf{T}^{\prime}$ que contienen conjuntamente a los vértices $x$ e $y$.

Demostración: En todos los casos la prueba se obtiene directamente a partir de la planaridad de $\mathbf{T}^{\prime}$. 


\section{Prueba del Teorema 5.4.1}

Demostración: Probaremos que si $\mathbf{T}^{\prime}$ es un grafo clique y no se satisfacen ni a) ni b), entonces vale c).

Si $\mathbf{T}^{\prime}$ es un grafo clique, existe una familia, $\mathcal{F}$, de completos de $\mathbf{T}^{\prime}$ que cubren sus aristas y tienen la propiedad Helly. Sea, como en el Lema 5.4.2, $u_{T} \in \cap \mathcal{F}_{T}$.

Si $u_{T} \in T$, podemos suponer sin pérdida de generalidad que $u_{T}=z$. Si no vale a), entonces $V_{x y} \neq \emptyset$. Sea $z_{1} \in V_{x y}$. Como $z_{1} \not z=u_{T}$ entonces (por Lema 5.4.2,1) $z_{1} \notin \mathbf{T}_{\mathcal{F}}$, entonces (por Lema 5.4.2,5) se debe cumplir: i) $V_{x y}=\left\{z_{1}\right\}, z_{1} \sim u \in V_{x y z}$, cual contradice la no validez de b); ó ii) $V_{x y}=\left\{z_{1}, z_{1}^{\prime}\right\}, z_{1} \sim z_{1}^{\prime} \sim u_{T} \in V_{x y z}$, lo cual contradice la suposición $u_{T}=z \in T$. En consecuencia, no puede ser $u_{T} \in T$.

Si $u_{T} \notin T$ debe ser (por Lema 5.4.2, 2) $u_{T} \in V_{x y z}$. Por Lema 5.4.2, 6, $V_{x y}, V_{x z}, \mathrm{y} V_{y z}$ contienen, cada uno de ellos, a lo sumo dos vértices. Supongamos que uno de ellos por ejemplo $V_{x y}$, tubiere solo uno: $z_{1}$. Como por hipótesis no vale b) $z_{1}$ no puede ser adyacente a ningún elemento de $V_{x y z}$, en particular no puede ser adyacente a $u_{T}$ pero entonces (por Lema 5.4.2,1) $z_{1} \notin \mathbf{T}_{\mathcal{F}}$, pero así (nuevamente Lema 5.4.2,5 ) es $z_{1} \sim u \in V_{x y z}$ lo cual dijimos que no es posible. Concluimos entonces que los tres conjuntos $V_{x y}, V_{x z}, \mathrm{y} V_{y z}$ tienen exactamente dos vértices. Como $u_{T} \in V_{x y z}$, en cada caso, (por planaridad) al menos uno de los dos vértices mencionados no puede ser adyacente a $u_{T}$ y por lo tanto no es un vértice de $T_{\mathcal{F}}$, entonces (por 5 del Lema 5.4.2) resulta c). Recordemos que el grafo descripto en c) es planar maximal, por lo tanto no puede haber mas aristas entre sus vértices.

La implicación recíproca dice que todo triángulo extendido planar que satisface a), b) o c) es un grafo clique. Es claro que todo triángulo extendido (planar o no) que satisface a) es un grafo clique pues tiene un vértice universal. Un triángulo extendido planar que satisface c) es isomorfo al grafo de la Figura 5.5, el cual claramente es grafo clique pues admite el siguiente cubrimiento por completos Helly:

$$
\begin{aligned}
& \left\{x, z_{1}, z_{1}^{\prime}\right\},\left\{y, z_{1}, z_{1}^{\prime}\right\},\left\{x, y, z_{1}^{\prime}, u\right\}, \\
& \left\{x, y_{1}, y_{1}^{\prime}\right\},\left\{z, y_{1}, y_{1}^{\prime}\right\},\left\{x, z, y_{1}^{\prime}, u\right\}, \\
& \left\{y, x_{1}, x_{1}^{\prime}\right\},\left\{z, x_{1}, x_{1}^{\prime}\right\},\left\{y, z, x_{1}^{\prime}, u\right\} .
\end{aligned}
$$




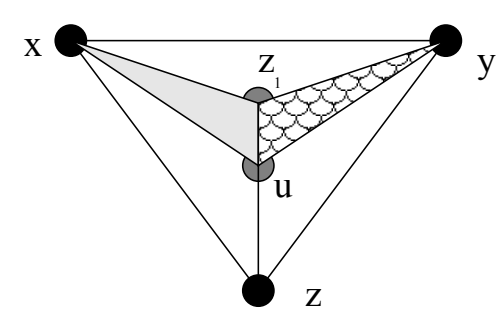

Figura 5.6: Cubrimiento en el Caso 1.

Resta considerar el caso de un triángulo extendido planar que satisface b). Sea el triangulo extendido planar $\mathbf{T}^{\prime}$, donde $T=\{x, y, z\}, V_{x y}=\left\{z_{1}\right\}, z_{1} \sim u \in V_{x y z}$, $V_{x z} \neq \emptyset$ y $V_{y z} \neq \emptyset$. Consideraremos cuatro casos: Caso 1: $V_{x y z}=\{u\}$, y $z_{1}$ es el único vértice de $V\left(\mathbf{T}^{\prime}\right)-T$ adyacente a $u$. Sea $\mathcal{F}$ la familia (Figura 5.6)

$$
\mathcal{F}=\left[\mathcal{C}\left(\mathbf{T}^{\prime}\right)-\left\{x, y, z_{1}, u\right\}\right] \cup\left\{x, z_{1}, u\right\} \cup\left\{y, z_{1}, u\right\}
$$

Es fácil ver que $\mathcal{F}$ es una familia de completos que cubre las aristas de $\mathbf{T}^{\prime}$, obsérvese que la arista $x y$ permanece cubierta por el clique $\{x, y, z, u\}$. Veamos que $\mathcal{F}$ satisface la propiedad Helly. Supongamos que $\mathcal{F}^{\prime}$ es una subfamilia mutuamente intersectante. Si todo miembro de $\mathcal{F}^{\prime}$ es un miembro de $\left[\mathcal{C}\left(\mathbf{T}^{\prime}\right)-\left\{x, y, z_{1}, u\right\}\right]$, por Lema 5.4.3, 3, todos ellos contienen el vértice $z$, por lo tanto $\cap \mathcal{F}^{\prime} \neq \emptyset$. Consideremos entonces que $\left\{x, z_{1}, u\right\} \in \mathcal{F}^{\prime}$, o $\left\{y, z_{1}, u\right\} \in \mathcal{F}^{\prime}$ (es el mismo caso por simetría), o ambos son miembros de $\mathcal{F}^{\prime}$ :

Subcaso 1.a: $F_{0}=\left\{x, z_{1}, u\right\} \in \mathcal{F}^{\prime}$ y $F_{1}=\left\{y, z_{1}, u\right\} \notin \mathcal{F}^{\prime}$. Por Lema 5.4.3, 1, $F_{0}$ es el único completo de $\mathcal{F}^{\prime}$ que contiene $z_{1}$, por lo tanto todo otro completo de $\mathcal{F}^{\prime}$ interseca a $F_{0}$ en $x$ o en $u$. Por Lema 5.4.3, 2 , el único completo, además de $F_{0}$, que contiene a $u$ es $\{x, y, z, u\}$ y este también contiene a $x$, concluimos entonces que todo miembro de $\mathcal{F}^{\prime}$ contiene el vértice $x$, por lo tanto $\cap \mathcal{F}^{\prime} \neq \emptyset$.

Subcaso 1.b: $F_{0}=\left\{x, z_{1}, u\right\} \in \mathcal{F}^{\prime}$ y $F_{1}=\left\{y, z_{1}, u\right\} \in \mathcal{F}^{\prime}$. Supongamos existe $F \in \mathcal{F}^{\prime}$ tal que $u \notin F$. Como $F_{0}$ y $F_{1}$ son los únicos completos que contienen a $z_{1}$, (Lemma 5.4.3, 1), y $\mathcal{F}^{\prime}$ es mutuamente intersectante, entonces $F \cap F_{0}=\{x\}$ y $F \cap F_{1}=\{y\}$, 


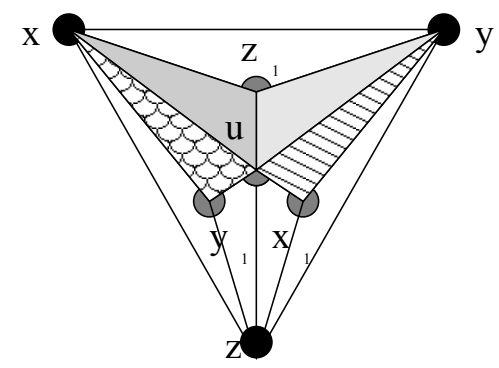

Figura 5.7: Cubrimiento en el Caso 2.

de donde los vértices $x, y$ pertencen a $F$, pero entonces por Lema 5.4.3, 4, debe ser $F=\{x, y, z, u\}$, lo cual contradice $u \notin F$. Concluimos que todo miembro de $\mathcal{F}^{\prime}$ contiene a $u$, por ende $\cap \mathcal{F}^{\prime} \neq \emptyset$.

Caso 2: $V_{x y z}=\{u\}$ y $z_{1}$ no es el único vértice de $V\left(\mathbf{T}^{\prime}\right)-T$ adyacente a $u$. Consideraremos sin pérdida de generalidad el caso en que existen vértices $y_{1} \in V_{x z} \mathrm{y} x_{1} \in V_{y z}$, adyacentes a $u$.

Llamemos $\mathbf{T}^{\prime \prime}$ al grafo que se obtiene quitando a $\mathbf{T}^{\prime}$ las aristas $x_{1} u$ y $y_{1} u$. $\mathbf{T}^{\prime \prime}$ es un triángulo extendido planar, relativo al mismo triángulo $T$, en la situación del caso 1 , entonces

$$
\mathcal{F}=\left[\mathcal{C}\left(\mathbf{T}^{\prime \prime}\right)-\left\{x, y, z_{1}, u\right\}\right] \cup\left\{x, z_{1}, u\right\} \cup\left\{y, z_{1}, u\right\}
$$

es una familia de completos que cubren las aristas de $\mathbf{T}^{\prime \prime}$ con la propiedad Helly. Ahora consideremos (Figura 5.7)

$$
\mathcal{F}_{1}=\mathcal{F} \cup\left\{x, y_{1}, u\right\} \cup\left\{x_{1}, y, u\right\}
$$

Es claro que $\mathcal{F}_{1}$ es una familia de completos de $\mathbf{T}^{\prime}$ que cubre sus aristas. Veamos que tiene la propiedad Helly. Sea $\mathcal{F}_{1}^{\prime}$ una subfamilia mutuamente intersectante de $\mathcal{F}_{1}$. Si $\mathcal{F}_{1}^{\prime}$ es subfamilia de $\mathcal{F}$ entonces tiene intersección no vacía; considereremos entonces que $\mathcal{F}_{1}^{\prime}$ contiene a $F_{2}=\left\{x, y_{1}, u\right\}$, o a $F_{3}=\left\{x_{1}, y, u\right\}$ (es el mismo caso por simetría), o a ambos:

Subcaso 2.a $: F_{2}=\left\{x, y_{1}, u\right\} \in \mathcal{F}_{1}^{\prime}$ y $F_{3}=\left\{x_{1}, y, u\right\} \notin \mathcal{F}_{1}^{\prime}$. Supongamos $\mathcal{F}_{1}^{\prime}$ tiene intersección vacía, entonces existe un miembro $B \in \mathcal{F}_{1}^{\prime}$ tal que $u \notin B$, por lo tanto $B$ 


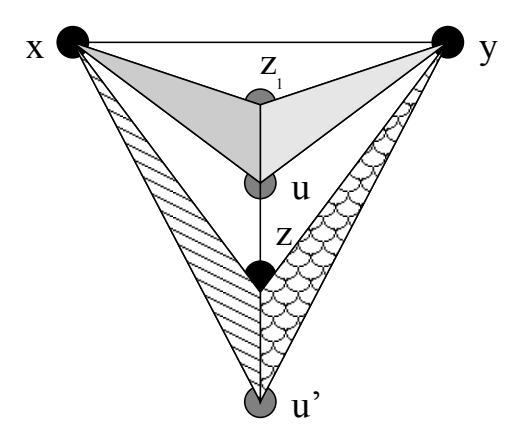

Figura 5.8: Cubrimiento en el Caso 3.

interseca $F_{2}$ en $x$ o en $y_{1}$. Como todo completo de $\mathcal{F}_{1}$ que contiene a $y_{1}$ contiene a $x$, deducimos que $B \supseteq\{x, \not \mu\}$.

Por otra parte, y por las mismas razones, debe existir un miembro $A \in \mathcal{F}_{1}^{\prime}$, tal que $x \notin A$, y por lo tanto $y_{1} \notin A$, además como $A$ interseca $F_{2}$, resulta $u \in A$. Los únicos miembros de $\mathcal{F}_{1}$ que satisfacen estas condiciones son $F_{3}=\left\{x_{1}, y, u\right\}$ (que no es un miembro de $\left.\mathcal{F}_{1}^{\prime}\right)$ y $F_{1}=\left\{y, z_{1}, u\right\}$, de donde resulta $A=F_{1}=\left\{y, z_{1}, u\right\}$.

Observemos ahora que $A=\left\{y, z_{1}, u\right\}$ y $B \supseteq\{x, \mu\}$ se intersecan, por lo tanto $y$ o $z_{1}$ están en $B$. Pero en ambos casos, si $x$, e $y$ o si $z_{1}$, y $x$ pertenecen a $B$, resulta que $u$ pertenece a $B$, lo cual es una contradicción. Concluimos que $\cap \mathcal{F}^{\prime} \neq \emptyset$.

Subcaso 2.b: $F_{2}=\left\{x, y_{1}, u\right\} \in \mathcal{F}_{1}^{\prime}$ y $F_{3}=\left\{x_{1}, y, u\right\} \in \mathcal{F}_{1}^{\prime}$. Supongamos entonces que existe un miembro $F \in \mathcal{F}_{1}^{\prime}$ tal que no contiene al vértice $u$. Como $F$ interseca a $F_{2}$ y a $F_{3}$, entonces $F$ contiene: $y$ e $y_{1}$ (no es posible), o $x$ y $x_{1}$ (no es posible), o $x_{1}$ e $y_{1}$ (no es posible), o, finalmente, $F$ contiene $x$ e $y$, en cuyo caso $F$ contiene a $u$, lo cual es una contradicción. Concluimos que todo otro miembro de $\mathcal{F}_{1}^{\prime}$ contiene a $u$.

Caso 3: $V_{x y z}=\left\{u, u^{\prime}\right\}$ y $z_{1}$ es el único vértice de $V\left(\mathbf{T}^{\prime}\right)-T$ adyacente a $u$.

Consideremos la familia (Figura 5.8):

$$
\begin{gathered}
\mathcal{F}=\left[\left[\mathcal{C}\left(\mathbf{T}^{\prime}\right)-\left\{x, y, z_{1}, u\right\}\right]-\left\{x, y, z, u^{\prime}\right\}\right] \cup\left\{x, z_{1}, u\right\} \cup \\
\cup\left\{y, z_{1}, u\right\} \cup\left\{x, z, u^{\prime}\right\} \cup\left\{y, z, u^{\prime}\right\} .
\end{gathered}
$$

Es claro que $\mathcal{F}$ cubre las aristas de $\mathbf{T}^{\prime}$, veamos que $\mathcal{F}$ satisface la propiedad Helly. Supongamos que $\mathcal{F}^{\prime}$ es una subfamilia mutuamente intersectante. Si todo miembro 


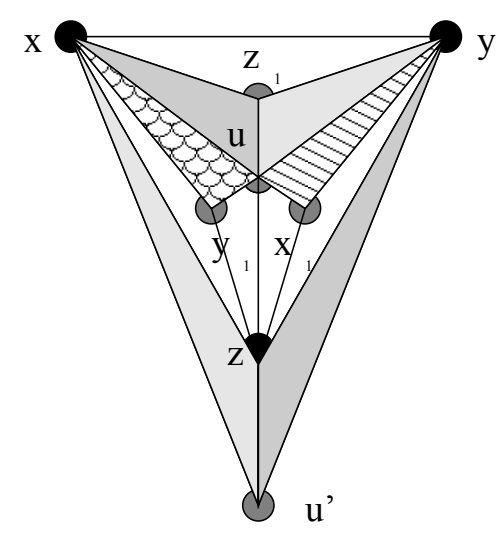

Figura 5.9: Cubrimiento en el Caso 4.

de $\mathcal{F}^{\prime}$ pertenece a $\left[\left[\mathcal{C}\left(T^{\prime}\right)-\left\{x, y, z_{1}, u\right\}\right]-\left\{x, y, z, u^{\prime}\right\}\right] \cup\left\{x, z, u^{\prime}\right\} \cup\left\{y, z, u^{\prime}\right\}$, entonces por Lema 5.4.3, 3, tienen al vértice $z$, en común. En caso en que $\left\{x, z_{1}, u\right\}$ o $\left\{y, z_{1}, u\right\}$ son miembos de $\mathcal{F}^{\prime}$, podemos repetir exactamente la demostración del caso 1 .

Caso 4: $V_{x y z}=\left\{u, u^{\prime}\right\}$ y $z_{1}$ no es el único vértice de $V\left(\mathbf{T}^{\prime}\right)-T$ adyacente a $u$. Nuevamente sin pérdida de generalidad podemos suponer existen vértices $y_{1} \in V_{x z} \mathrm{y}$ $x_{1} \in V_{y z}$ adyacentes a $u$.

Consideremos $\mathbf{T}^{\prime \prime}$, como el grafo $\mathbf{T}^{\prime}$ sin las aristas $x_{1} u$ e $y_{1} u . \mathbf{T}^{\prime \prime}$ es un triángulo extendido planar en las condiciones del caso 3. Llamemos $\mathcal{F}$ el cubrimiento por completos Helly de $\mathbf{T}^{\prime \prime}$ indicado en ese caso. Ahora consideremos la familia (Figura $5.9)$ :

$$
\mathcal{F}_{1}=\mathcal{F} \cup\left\{x, y_{1}, u\right\} \cup\left\{x_{1}, y, u\right\}
$$

Es claro que $\mathcal{F}_{1}$ es un cubrimiento por completos de las aristas de $\mathbf{T}^{\prime}$. Veamos que es Helly. Si $\mathcal{F}_{1}^{\prime}$ es una subfamilia de mutuamente intersectante de $\mathcal{F}_{1}$ y está contenida en $\mathcal{F}$, claramente tiene intersección no vacía. Si no, $\mathcal{F}_{1}^{\prime}$ debe contener a $F_{2}=\left\{x, y_{1}, u\right\}$ o a $F_{3}=\left\{x_{1}, y, u\right\}$, o a ambos, y en esta situación podemos repetir exatamente la demostración hecha en el caso 2. 


\section{Complejidad Algorítmica}

La caracterización dada en esta sección, Teorema 5.4.1, permite implementar un algoritmo eficiente de reconocimiento de los triángulos extendidos de un grafo planar, que son grafos clique.

Por otra parte podemos observar lo siguiente: supongamos que debemos determinar si una grafo planar dado, G, es o no grafo clique. De acuerdo a lo expuesto precedentemente podemos chequear eficientemente, a partir de la caracterización que presentamos en esta sección, si cada uno de los triángulos extendidos de $\mathbf{G}$ es un grafo clique y en caso de encontrar un triángulo extendido que no sea grafo clique, sabremos que $\mathbf{G}$ no es un grafo clique. Esta tarea puede también ser simplificada, pues no necesariamente habrá que recorrer todos los triángulos de G; veamos: si $T$ es un triángulo de $\mathbf{G}$ tal que $\mathbf{T}^{\prime}$ es grafo clique, entonces para todo triángulo $T_{1}$ tal que $\mathbf{T}_{1}^{\prime} \subseteq \mathbf{T}^{\prime}$ es inmediato que $\mathbf{T}_{1}^{\prime}$ es grafo clique, pues en la circunstancias descriptas, el triángulo extendido del grafo $\mathbf{T}^{\prime}$ relativo al triángulo $T_{1}$ es el mismo $\mathbf{T}_{1}^{\prime}$, entonces siendo $\mathbf{T}^{\prime}$ grafo clique (por el Corolario 5.3.2) debe serlo $\mathbf{T}_{1}^{\prime}$. Por lo tanto la tarea de chequear si los triángulos extendidos de $\mathbf{G}$ son grafos clique, no debe necesariamente ser desarrollada sobre todos los triángulos de $\mathbf{G}$. 


\section{Capítulo 6}

\section{Asignación Triángulo-Vértice}

Como hemos visto en el Capítulo 4 un grafo es grafo clique si y solo si existe una familia de completos que cubre las arista del grafo, tal que para todo triángulo existe un vértice común a todos los miembros de la familia pegados a él. Por lo tanto si $\mathbf{G}$ es un grafo clique, dada $\mathcal{F}$ una familia $\mathcal{R}-\mathcal{S}$, se puede vincular o relacionar a cada triángulo $T$ de $\mathbf{G}$ con un vértice particular.

Para cada $T \in T(\mathbf{G})$, existe $u_{T} \in V(\mathbf{G})$ tal que $u_{T} \in \cap \mathcal{F}_{T}$.

Por otra parte observamos, en las distintas demostraciones realizadas en los capítulos anteriores para el caso de los grafos planares, la importancia del papel desarrollado por el vértice $u_{T}$ y la cantidad de propiedades que este vértice tiene; es decir, la existencia de una familia $\mathcal{R}-\mathcal{S}$ del grafo, destaca con propiedades particulares a un vértice en torno a cada triángulo.

En este capítulo trabajeremos sobre la idea recíproca: mostraremos que si a cada triángulo del grafo se le puede asignar un vértice, respetando ciertas condiciones, es posible construir a partir de estos vértices una familia $\mathcal{R}-\mathcal{S}$ del grafo.

Obtendremos además una nueva caracterización de los grafos cliques, la cual consideramos será una nueva herramienta para la resolución del problema del reconocimiento de los grafos clique. 
Finalmente presentaremos ejemplos de aplicación.

Tal como en el capítulo anterior, sin pérdida de generalidad, consideraremos grafos tales que sus cliques contienen al menos tres vértices.

\subsection{Definiciones}

Una asignación triángulo-vértice de un grafo $\mathbf{G}$, es una función $\mathbf{u}: T(\mathbf{G}) \rightarrow V(\mathbf{G})$, (para simplificar la notación en lo que sigue denotaremos $\mathbf{u}(T)=u_{T}$ ), tal que para todo triángulo $T=\{x, y, z\}$ de $\mathbf{G}$ se satisface una de las siguientes condiciones:

1. $u_{T} \in T$

$\mathrm{O}$

2. $u_{T} \in V_{x y z}$ y en este caso $u_{T_{1}}=u_{T_{2}}=u_{T_{3}}=u_{T}$, donde $T_{1}=\left\{y, z, u_{T}\right\}, T_{2}=\left\{x, z, u_{T}\right\}$ y $T_{3}=\left\{x, y, u_{T}\right\}$.

$\mathrm{X}$

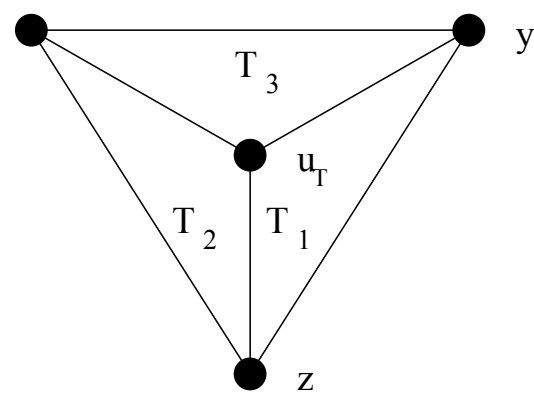

Figura 6.1:

El conjunto de los triángulos de $\mathbf{G}$ para los cuales $u_{T} \in T$ se denotará $T(\mathbf{G})_{\mathbf{u}}$. A tales triángulos les llamaresmo propios, por cuanto se les asigna un vértice que les pertenece.

$$
T(\mathbf{G})_{\mathbf{u}}=\left\{T \in T(\mathbf{G}) / u_{T} \in T\right\}
$$

Veamos que dada una asignación triángulo-vértice, toda arista está cubierta por un triángulo propio. 
Proposición 6.1.1. Si $\mathbf{u}$ es una asignación triángulo-vértice de un grafo $\mathbf{G}$ y uv $\in$ $E(\mathbf{G})$ entonces existe $T \in T(\mathbf{G})_{\mathbf{u}}$ que $\{u, v\} \subseteq T$.

Demostración: Como todo clique contiene al menos tres vértices, toda arista uv está cubierta por algún triángulo $T$. Si $u_{T} \in T$, claramente vale la proposición. Si $u_{T} \notin T$, por la condición 2 pedida en la definición de asignación triángulo-vértice, resulta que $u_{\left\{u, v, u_{T}\right\}}=u_{T}$, de donde $T_{1}=\left\{u, v, u_{T}\right\}$ cubre la arista $u v$ y $u_{T_{1}} \in T_{1}$.

Dada $\mathbf{u}$, una asignación triángulo-vértice de un grafo $\mathbf{G}, V^{\prime}$ un subconjunto de vértices de $\mathbf{G}$, y $s$ un número entero positivo, llamaremos $A_{\mathbf{u}}^{s}\left(V^{\prime}\right)$ al subconjunto de vértices de $\mathbf{G}$, definido de la siguiente forma:

$$
\begin{aligned}
A_{\mathbf{u}}^{1}\left(V^{\prime}\right) & =V^{\prime} \cup\left\{u_{T}, T \in T(\mathbf{G}), T \text { pegado a } V^{\prime}\right\} \\
& =V^{\prime} \cup\left\{u_{T}, T \in T(\mathbf{G})_{\mathbf{u}}, T \text { pegado a } V^{\prime}\right\} . \\
A_{\mathbf{u}}^{s}\left(V^{\prime}\right) & =A^{1}\left(A_{\mathbf{u}}^{s-1}\left(V^{\prime}\right)\right) .
\end{aligned}
$$

Recordemos que $T$ pegado a $V^{\prime}$ significa que $\left|T \cap V^{\prime}\right| \geq 2$ e interpretemos como se forman los conjuntos definidos: partimos del conjunto de vértices $V^{\prime}$, agregamos a $V^{\prime}$ los vértices $u_{T}$ asignados a los triángulos $T$ pegados a $V^{\prime}$, así obtenemos $A_{\mathbf{u}}^{1}\left(V^{\prime}\right)$. Por la condición 2 de la definición de asignación triángulo-vértice es claro que es lo mismo agregar solo los $u_{T}$ asignados a triángulos $T$ pertenecientes a $T(\mathbf{G})_{\mathbf{u}}$.

Ahora agregamos a $A_{\mathbf{u}}^{1}\left(V^{\prime}\right)$ los vértices $u_{T}$ correspondientes a los triángulos $T$ pegados $A_{\mathbf{u}}^{1}\left(V^{\prime}\right)$, así obtenemos $A_{\mathbf{u}}^{2}\left(V^{\prime}\right)$. De esta forma podríamos continuar indefinidamente, pero dado que estos conjuntos de vértices pueden crecer a lo sumo hasta ser igual a $V(\mathbf{G})$, existe un entero positivo, que notaremos $s_{V^{\prime}}$, tal que

$$
A_{\mathbf{u}}^{s_{V^{\prime}}}\left(V^{\prime}\right)=A_{\mathbf{u}}^{s_{V^{\prime}}+1}\left(V^{\prime}\right)
$$


Vale entonces que para todo entero $s, s \geq s_{V^{\prime}}$

$$
A_{\mathbf{u}}^{s}\left(V^{\prime}\right)=A_{\mathbf{u}}^{s_{V^{\prime}}}\left(V^{\prime}\right)
$$

Llamaremos engordado de $V^{\prime}$ según la asignación triángulo-vértice $\mathbf{u}$, al conjunto de vértices:

$$
A_{\mathbf{u}}\left(V^{\prime}\right)=A_{\mathbf{u}}^{s_{V^{\prime}}}\left(V^{\prime}\right)
$$

El conjunto $A_{\mathbf{u}}\left(V^{\prime}\right)$ ya no crece por el agregado de vértices $u_{T}$, sino que se ha estabilizado, esto se expresa claramente en la siguiente proposición.

Proposición 6.1.2. Si $T$ es un triángulo pegado a $A_{\mathbf{u}}\left(V^{\prime}\right)$ entonces el vértice $u_{T}$ pertenece a $A_{\mathbf{u}}\left(V^{\prime}\right)$.

Teorema 6.1.1. Sea u una asignación triángulo-vértice de un grafo $\mathbf{G}$. Si para todo triángulo propio, $T \in T(\mathbf{G})_{\mathbf{u}}, A_{\mathbf{u}}(T)$ es un completo de $\mathbf{G}$ entonces

$$
\left(A_{\mathbf{u}}(T)\right)_{T \in T(\mathbf{G})_{\mathbf{u}}} \text { es una familia } \mathcal{R}-\mathcal{S} \text { de } \mathbf{G}
$$

Demostración: Llamemos $\mathcal{F}$ a la familia $\left(A_{\mathbf{u}}(T)\right)_{T \in T(\mathbf{G})_{\mathbf{u}}}$. Por hipótesis todo miembro de $\mathcal{F}$ es un completo; por otra parte se deduce de la Proposición 6.1.1 que los miembros de $\mathcal{F}$ cubren las aristas de $\mathbf{G}$. Para demostrar que $\mathcal{F}$ satisface la propiedad de Helly, probaremos que se satisface el Lema 5.2.2, es decir probaremos que para todo triángulo $T$ de $\mathbf{G}$, la interseción de todos los miembros de $\mathcal{F}$ pegados a $T$ es no vacía, más precisamente probaremos que $u_{T}$ pertenece a esa intersección.

Sea $T$ un triángulo de $\mathbf{G}$ y $A_{\mathbf{u}}(\bar{T})$ un miembro de $\mathcal{F}$ pegado a $T$. Se deduce de la Proposición 6.1.2 que $u_{T} \in A_{\mathbf{u}}(\bar{T})$, como queríamos probar.

Observemos lo siguiente: sabemos que determinar la existencia o no de una familia $\mathcal{R}-\mathcal{S}$ de un grafo dado es una tarea sumamente complicada. El teorema anterior dice 
que en lugar de buscar una familia $\mathcal{R}-\mathcal{S}$ se puede buscar una asignación triángulovértice tal que los engordados de los triángulos propios sean completos. Aspiramos a que en futuros trabajos, al menos para determinadas clases de grafos, la condición de completitud de los triángulos engordados, imponga suficientes condiciones a los vértices a asignar a cada triángulo, como para que sea posible determinar facilmente la existencia o no de una tal asignación.

Haremos al final de este capítulo algunos ejemplos para ampliar estas ideas.

Veamos ahora como toda familia $\mathcal{R}-\mathcal{S}$ define una asignación triángulo vértice y como los engordados de los triángulo por esa asignación guardan información de la familia $\mathcal{R}-\mathcal{S}$ original.

Lema 6.1.2. Dada $\mathcal{F}$ una familia $\mathcal{R}-\mathcal{S}$ de un gafo $\mathbf{G}$, elijamos para cada $T \in$ $T(\mathbf{G})$, un vértice $u_{T}$ tal que $u_{T} \in \cap \mathcal{F}_{T}$ y siempre que sea posible $u_{T} \in T$. La aplicación $\mathbf{u}: T(\mathbf{G}) \rightarrow V(\mathbf{G})$, tal que $\mathbf{u}(T)=u_{T}$ es una asignación triángulo-vértice de $\mathbf{G}$, tal que para todo $T \in T(\mathbf{G})$, para todo $F \in \mathcal{F}$,

$$
T \subseteq F \Longrightarrow A_{\mathbf{u}}(T) \subseteq F
$$

Más aún, $\left(A_{\mathbf{u}}(T)\right)_{T \in T(\mathbf{G})_{\mathbf{u}}}$ es otra familia $\mathcal{R}-\mathcal{S}$ del grafo $\mathbf{G}$.

Demostración: Si $\mathcal{F}$ es una familia $\mathcal{R}-\mathcal{S}$ de $\mathbf{G}$ entonces para todo triángulo $T$ de $\mathbf{G}, \cap \mathcal{F}_{T} \neq \emptyset$ (Lema 5.2.2). Se deduce que para todo triángulo $T$ de $\mathbf{G}$ es posible elegir un vértice $u_{T}$ satisfaciendo lo pedido en el enunciado.

Veamos que la aplicación $\mathbf{u}(T)=u_{T}$, es una asignación triángulo-vértice de $\mathbf{G}$. Sea $T=\{x, y, z\}$ un triángulo de $\mathbf{G}$, tal que $u_{T} \notin T$. Como toda arista de $T$ está cubierta por algún miembro de $\mathcal{F}$, y como $u_{T}$ pertenece a todos los miembros de $\mathcal{F}$ pegados a $T$, se deduce $u_{T} \in V_{x y z}$.

Consideremos los triángulos $T_{1}=\left\{y, z, u_{T}\right\}, T_{2}=\left\{x, z, u_{T}\right\}$ y $T_{3}=\left\{x, y, u_{T}\right\}$, debemos mostrar que $u_{T_{1}}=u_{T_{2}}=u_{T_{3}}=u_{T}$. Por simetría es suficiente probar que $u_{T_{1}}=u_{T}$ y esto se deduce directamente de la forma en que fue elegido $u_{T_{1}} \mathrm{y}$ de los siguientes hechos: 


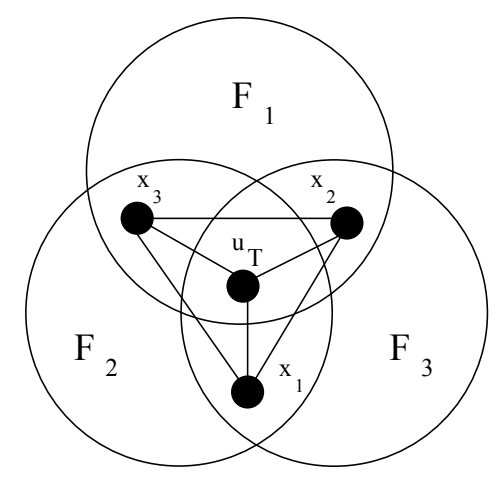

Figura 6.2: Los miembros $F_{i}, 1 \leq i \leq 3$

I) $u_{T} \in T_{1}$.

II) $u_{T} \in \cap \mathcal{F}_{T_{1}}$ pues si $F \in \mathcal{F}_{T_{1}}$, entonces $F$ es un miembro de la familia $\mathcal{F}$ pegado a $T_{1}$, por lo tanto contiene dos vértices de $T_{1}$. Si uno de estos vértices es $u_{T}$, listo, sino $F$ debe contener a los vértices y y $z$, de donde $F$ está pegado a $T$, y por lo tanto $F \in \mathcal{F}_{T}$ y así resulta $u_{T} \in F$.

III) $u_{T_{1}}$ no puede ser $y$ ni $z$, pues, como probamos a continuación, estos vértices no pertenecen a $\cap \mathcal{F}_{T_{1}}$.

Como $u_{T} \notin T$ entonces $\cap \mathcal{F}_{T} \cap T=\emptyset$, pues en otro caso el vértice $u_{T}$ hubiera sido elegido perteneciente a $T$. Entonces, deben existir miembros de $\mathcal{F}$, (Figura 6.2):

$$
\begin{aligned}
& F_{1} \supseteq\left\{y, z, u_{T}, \not x\right\}, \\
& F_{2} \supseteq\left\{x, z, u_{T}, \not y\right\}, \\
& F_{3} \supseteq\left\{x, y, u_{T}, \not z\right\} .
\end{aligned}
$$

Claramente $F_{2} \in \mathcal{F}_{T_{1}}$ e $y \notin F_{2}$, entonces $y \neq u_{T_{1}}$. Análogamente, $F_{3} \in \mathcal{F}_{T_{1}}$ y $z \notin F_{3}$, entonces $z \neq u_{T_{1}}$.

Hemos probado que $\mathbf{u}$ es una asignación triángulo-vértice de $\mathbf{G}$.

Sea $\bar{T} \in T(\mathbf{G})$ y $F \in \mathcal{F}$ tal que $\bar{T} \subseteq F$. Probaremos por inducción sobre $s$ que $A_{\mathbf{u}}^{s}(\bar{T}) \subseteq F$, con lo cual $A_{\mathbf{u}}(\bar{T}) \subseteq F$. 
Sea $\mathrm{s}=1$,

$$
A_{\mathbf{u}}^{1}(\bar{T})=\bar{T} \cup\left\{u_{T}, T \text { pegado a } \bar{T}\right\}
$$

Veamos que si $T$ es un triángulo pegado a $\bar{T}$ entonces $u_{T} \in F$. Como por hipótesis $\bar{T} \subseteq F$, entonces $T$ y $F$ están pegados, i.e. $F \in \mathcal{F}_{T}$ por lo tanto $u_{T} \in F$.

La hipótesis inductiva dice que

$$
A_{\mathbf{u}}^{k}(\bar{T}) \subseteq F
$$

Sea $s=k+1$,

$$
A_{\mathbf{u}}^{k+1}(\bar{T})=A_{\mathbf{u}}^{1}\left(A_{\mathbf{u}}^{k}(\bar{T})\right)=A_{\mathbf{u}}^{k}(\bar{T}) \cup\left\{u_{T}, T \text { pegado a } A_{\mathbf{u}}^{k}(\bar{T})\right\}
$$

Veamos que si $T$ está pegado a $A_{\mathbf{u}}^{k}(\bar{T})$, entonces $u_{T} \in F$. Si $T$ está pegado a $A_{\mathbf{u}}^{k}(\bar{T})$,

como por hipótesis inductiva $A_{\mathbf{u}}^{k}(\bar{T}) \subseteq F$, entonces $T$ está pegado a $F$, i.e. $F \in \mathcal{F}_{T}$, de donde se deduce que $u_{T} \in F$.

Como hemos demostrado que $\mathbf{u}$ es una asignación triángulo-vértice, en vista del Teorema 6.1.1, para demostrar que $\left(A_{\mathbf{u}}(T)\right)_{T \in T(\mathbf{G})_{\mathbf{u}}}$ es una familia $\mathcal{R}-\mathcal{S}$, basta ver que si $T$ es un triángulo propio, entonces $A_{\mathbf{u}}(T)$ es un completo de $\mathbf{G}$. Por cierto, si $T \in T(\mathbf{G})_{\mathbf{u}}$ entonces existe $F \in \mathcal{F}$ tal que $T \subseteq F$, por lo tanto de acuerdo a lo demostrado anteriormente $A_{\mathbf{u}}(T) \subseteq F$, de donde $A_{\mathbf{u}}(T)$ es un completo de $\mathbf{G}$ como queríamos probar.

\subsection{Una nueva caracterización de los Grafos Clique}

Teorema 6.2.1. Un grafo $\mathbf{G}$ es un grafo Clique si y solo si existe $\mathbf{u}$, asignación triángulo-vértice de $\mathbf{G}$, tal que para todo triángulo propio $T \in T(\mathbf{G})_{\mathbf{u}}$, se verifica que $A_{\mathbf{u}}(T)$ es un completo de $\mathbf{G}$. 
Demostración: Sea $\mathbf{G}$ un grafo Clique. Por Teorema 2.4.1 existe $\mathcal{F}$, una familia $\mathcal{R}-\mathcal{S}$ de $\mathbf{G}$. Sea u una asignación triángulo-vértice de $\mathbf{G}$ obtenida a partir de $\mathcal{F}$ como en el Lema 6.1.2; tenemos que probar que si $u_{T} \in T$ entonces $A_{\mathbf{u}}(T)$ es un completo de G. Si $u_{T} \in T$ es claro que existe $F$, miembro de $\mathcal{F}$, tal que $T \subseteq F$, entonces, como el Lema 6.1.2 se satisface, $A_{\mathbf{u}}(T) \subseteq F$, por lo tanto, como $F$ es un completo, $A_{\mathbf{u}}(T)$ es un completo de $\mathbf{G}$.

La implicación recíproca es inmediata a partir del Lema 6.1.1.

Teorema 6.2.2. Un grafo $\mathbf{G}$ es un grafo clique si y solo si existe $\mathbf{u}$, asignación triángulo-vértice de $\mathbf{G}$, tal que $A_{\mathbf{u}}($ e) es un completo de $\mathbf{G}$ para toda arista $e \in E(\mathbf{G})$.

Demostración: Sea $\mathbf{G}$ un grafo clique y $\mathbf{u}$ una asignación triángulo-vértice de $\mathbf{G}$ satisfaciendo el Teorema 6.2.1. Sea $e$ una arista de G. Si todo triángulo $T$ pegado a $e$, es tal que $u_{T} \in e$, entonces $A_{\mathbf{u}}(e)=e$, por lo tanto es un completo. Si existe un triángulo $\bar{T}$ pegado a $e$ y $u_{\bar{T}} \notin e$, entonces existe un triángulo $T$ pegado a $e$ tal que $u_{T} \in T$ y $u_{T} \notin e,(T$ puede ser el mismo $\bar{T})$. Es fácil probar que $A_{\mathbf{u}}(e)=A_{\mathbf{u}}(T) \mathrm{y}$ como por Teorema 6.2.1, $A_{\mathbf{u}}(T)$ es un completo de $\mathbf{G}$, la demostración se completa. Recíprocamente, sea u una asignación triángulo-vértice de $\mathbf{G}$ que satisface la hipótesis. Sea $T$ un triángulo de $\mathbf{G}$ tal que $u_{T} \in T$. Sea $e$ la arista de $T$ tal que $u_{T}$ no es un vértice extremo de $e$. Es claro que $A_{\mathbf{u}}(e)=A_{\mathbf{u}}(T)$, entonces $A_{\mathbf{u}}(T)$ es un completo, se deduce entonces, a partir del teorema anterior, que $\mathbf{G}$ es un grafo clique. 


\subsection{Ejemplos de Aplicación}

Diremos que una asignación triángulo-vértice, $\mathbf{u}$, de un grafo $\mathbf{G}$, es buena si para todo $T \in T(\mathbf{G})_{\mathbf{u}}$ se verifica que $A_{\mathbf{u}}(T)$ es un completo de $\mathbf{G}$.

\section{Condiciones a priori sobre los $u_{T}$}

Supongamos queremos determinar si un grafo es o no grafo clique utilizando los resultados de este capítulo. Una forma podría ser considerar todas las posibles asignaciones triángulo vértice y ver si alguna de ellas es buena, es decir si los $A_{\mathbf{u}}$ son completos. Esta es, sin embargo, una tarea difícil pues la cantidad de asignaciones triángulo vértice puede ser muy grande, de hecho cada triángulo admite al menos tres candidados a $u_{T}$.

Otra forma de trabajar, la cual creemos será de utilidad, es ir construyendo una buena asignación a partir de un triángulo. Es decir, en principio cada triángulo $\mathrm{T}$ admite como candidato a $u_{T}$, cualquiera de sus tres vértices y cualquier vértice adyacente a estos tres. Comenzando por un triángulo cualquiera dado, se le asigna a éste un vértice, esto inmediatamente influye sobre los posibles vértices a asignar a los triángulos pegados al primero, por la condición de ir conformando un completo del grafo. Así se puede ir avanzando sobre los demás triángulos como a lo largo de un árbol. Cuando no es posible asignar un vértice a un triángulo se vuelve y se cambia el asignado a un triángulo anterior.

Solo a modo de nota marginal, quisieramos destacar la similitud que este problema tiene con el de Satisfabilidad, lo que sugiere que el problema de reconocimiento de los grafos en $K$ (Grafos) podría ser NP-completo.

Por otra parte se pueden desarrollar a priori condiciones que deben cumplir los $u_{T}$, disminuyendo de esta forma la cantidad de vértices candidatos. Por ejemplo, supong-

amos que $T=\{x, y, z\}$ es un clique del grafo. En cualquier asignación este triángulo ha de ser propio pues $V_{x y z}=\emptyset$. Se deduce inmediatamente que cualquier triángulo pegado a $T$, tiene como únicos candidatos a ser asignados en una buena asignación 
triángulo-vértice, solo dos de los vértices de $T$. Para hacer más clara la explicación consideremos el triángulo $T_{1}$ pegado a $T$, de la siguiente figura.

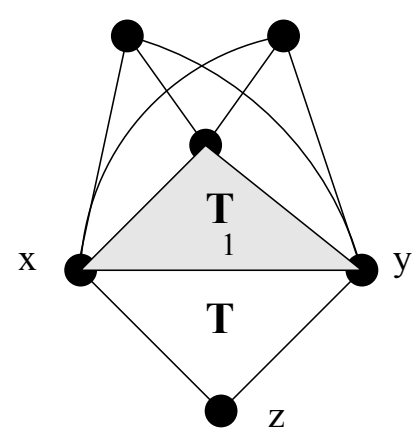

Claramente existen 5 vértices que podrían asignarse a $T_{1}$, pero en una buena asignación los candidatos son solo dos: $x$ e $y$, independientemente de lo que se haga en otros triángulos.

Obtenemos así lo que podemos llamar una regla de buena asignación:

Regla 1: Si $T$ es un clique y $T_{1}$ está pegado a $T$ entonces $u_{T_{1}} \in T \cap T_{1}$.

Fácilmente se prueba la siguiente regla.

Regla 2: $S i T_{1}$ y $T_{2}$ está pegados, entonces $u_{T_{1}}=u_{T_{2}}$ o $u_{T_{1}} \sim u_{T_{2}}$.

\section{La Pirámide no es grafo clique}

Enumeremos los triángulos de la Pirámide como en la siguiente figura.

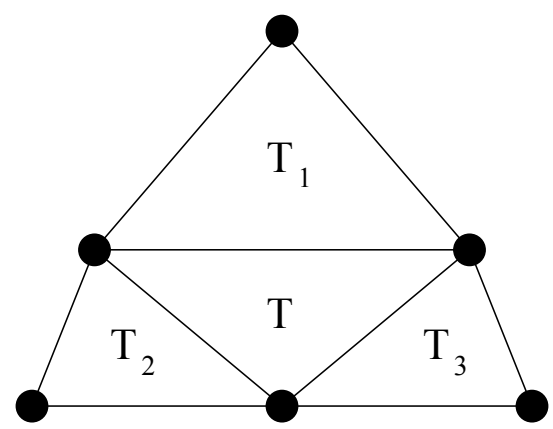


Es inmediato a partir de la Regla 1 que la Pirámide no es grafo clique pues en una buena asignación $u_{T}$ debería pertenecer a $T_{1} \cap T$, a $T_{2} \cap T$, y a $T_{1} \cap T$, lo cual no es posible.

\section{La Pirámide de dos cabezas no es grafo clique}

Consideremos el grafo de la siguiente figura al que llamamos Pirámide de dos cabezas.

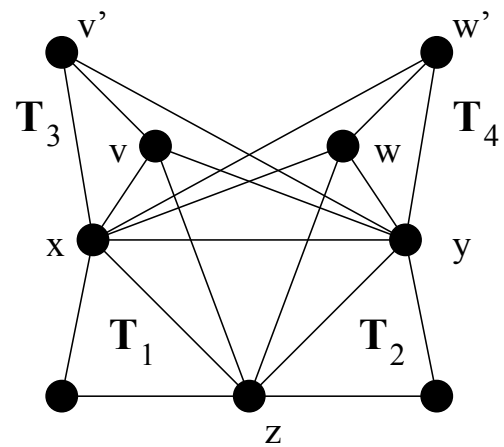

$$
T=\{x, y, z\}, T_{3}=\left\{x, y, v^{\prime}\right\} \text { y } T_{4}=\left\{x, y, w^{\prime}\right\}
$$

Supongamos existe $\mathbf{u}$ una buena asignación triángulo-vértice de este grafo.

Como los triángulos $T_{1}$ y $T_{2}$ son cliques del grafo, por la Regla 1, debe ser $u_{T}=z$.

El engordado de $T_{3}$ contiene a $T_{3}$ y a $u_{T}=z$, como no se forma un completo, $T_{3}$ no puede ser un triángulo propio de $\mathbf{u}$, es decir $u_{T_{3}} \notin T_{3}$ y por lo tanto la única posibilidad es $u_{T_{3}}=v$ y así además $u_{\{x, y, v\}}=v$.

Análogamente, $u_{T_{4}} \notin T_{4}$, de donde $u_{T_{4}}=w$. Además $u_{\{x, y, w\}}=w$.

Finalmente $T_{3}$ y $T_{4}$ están pegados pero $u_{T_{3}}$ y $u_{T_{4}}$ no son adyacentes, lo cual contradice la Regla 2 .

\section{Reglas para clases particulares de grafos}

Como hemos visto en el primer ejemplo, se pueden desarrollar reglas que disminuyan la cantidad de vértices candidatos a ser asignados a un triángulo. En este ejemplo veremos que también se puede trabajar en el desarrollo de reglas para clases particulares 
de grafos.

Regla para planares: Sea $T=\{x, y, z\}$ un triángulo de un grafo planar G. Si $\left|V_{x y z}\right| \geq 2$ entonces $T$ es un triángulo propio en cualquier buena asignación de $\mathbf{G}$.

Demostración: Sea u una buena asignación de G y supongamos $T$ no es propio, entonces $u_{T} \in V_{x y z}$. Sea $w \in V_{x y z}, w \neq u_{T}$. Llamemos $T_{1}=\{y, z, w\}$.

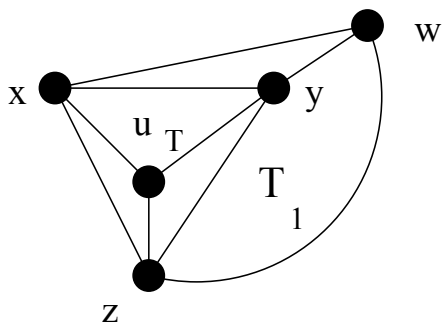

Como $\mathbf{G}$ planar $u_{T} \not w, u_{T_{1}} \neq u_{T}$ y además, por Regla $1, T_{1}$ no puede ser propio. Por otra parte $u_{T_{1}}$ no puede ser $x$, pues sino por la condición 2 de la definición de asignación triángulo-vértice sería $u_{T}=x$. Se deduce entonces que $u_{T_{1}}$ es un vértice distinto de los ya descriptos.

Como $T$ y $T_{1}$ están pegados, por la Regla 2 , debe ser $u_{T} \sim u_{T_{1}}$, con lo cual la situación es la descripta en la siguiente figura, lo cual contradice la planaridad de $\mathbf{G}$.

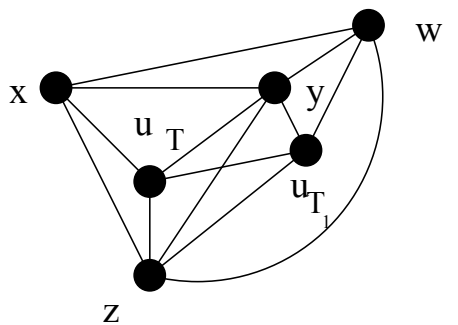




\section{Los grafos A y B}

En [11] se dice que un grafo tiene la Pirámide si existe tres vértices mutuamente adyacentes, $x, y, z$ y vértices $x_{1}, y_{1}, z_{1}$ tales que

$$
\begin{array}{lll}
x_{1} \sim y & x_{1} \sim z & x_{1} \not x \\
y_{1} \sim x & y_{1} \sim z & y_{1} \not y \\
z_{1} \sim x & z_{1} \sim y & z_{1} \not z
\end{array}
$$

Obsérvese que con la terminología introducida en el capítulo 4, esto es equivalente a decir que el grafo tiene un triángulo $T$ tal que $\mathbf{T}^{\prime}$ no es de tipo 1 . Por otra parte si todo triángulo es tal que $\mathbf{T}^{\prime}$ es de tipo 1 entonces el grafo dado es grafo clique.

En ese mismo artículo se muestra que si $\mathbf{G} \in K(\mathbf{G r a f o s})$ y $\mathbf{G}$ tiene la Pirámide entonces $\mathbf{G}$ tiene un subgrafo isomorfo a $\mathbf{A}$ (Figura 5.3) o a B (Figura 5.5). Además se ve que no vale el resultado recíproco pues se muestra como contraejemplo el caso de la Pirámide de dos cabezas que tiene dos veces a $\mathbf{A}$ pero no es grafo clique.

Con los resultados de este capítulo podemos explicar mejor que ocurre con los grafos clique que tienen a la Pirámide.

Sea $T=\{x, y, z\} \in T(\mathbf{G})$ tal que $\mathbf{T}^{\prime}$ no es de tipo 1. Sea u una buena asignación de G. Hay dos posibilidades:

I: $u_{T} \in T$, por ejemplo $u_{T}=z$, y entonces:

i) Para todo vértice $z_{1} \in V_{x y}$ debe existir un vértice $z_{1}^{\prime} \in V_{x y z_{1}}$ adyacente a $z$. Y más aún,

ii) $x, y, z$ y todos los vértices $z_{1}^{\prime}$ correspondientes a los vértices $z_{1}$ de $V_{x y}$, deben conformar un completo de $\mathbf{G}$.

O bien, II: $u_{T} \in V_{x y z}$ y en este caso,

i)Para todo vértice $z_{1} \in V_{x y}, z_{1} \sim u_{T}$ o existe otro vértice $z_{1}^{\prime} \in V_{x y z_{1}}$ tal que $z_{1}^{\prime} \sim u_{T}$.

ii)Simétricamente igual a i) para todo vértice $y_{1} \in V_{x z}$.

ii)Simétricamente igual a i) para todo vértice $z_{1} \in V_{x y}$.

Se podría agregar también la condición de completitud, pero a los fines de este ejemplo lo consideramos innecesario. 
Los siguientes son ejemplos de grafos clique en los que aparece la pirámide y por lo tanto aparecen los subgrafos A o B, según ocurra el caso I o II recién descriptos.

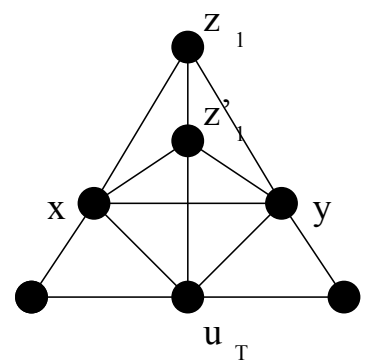

A
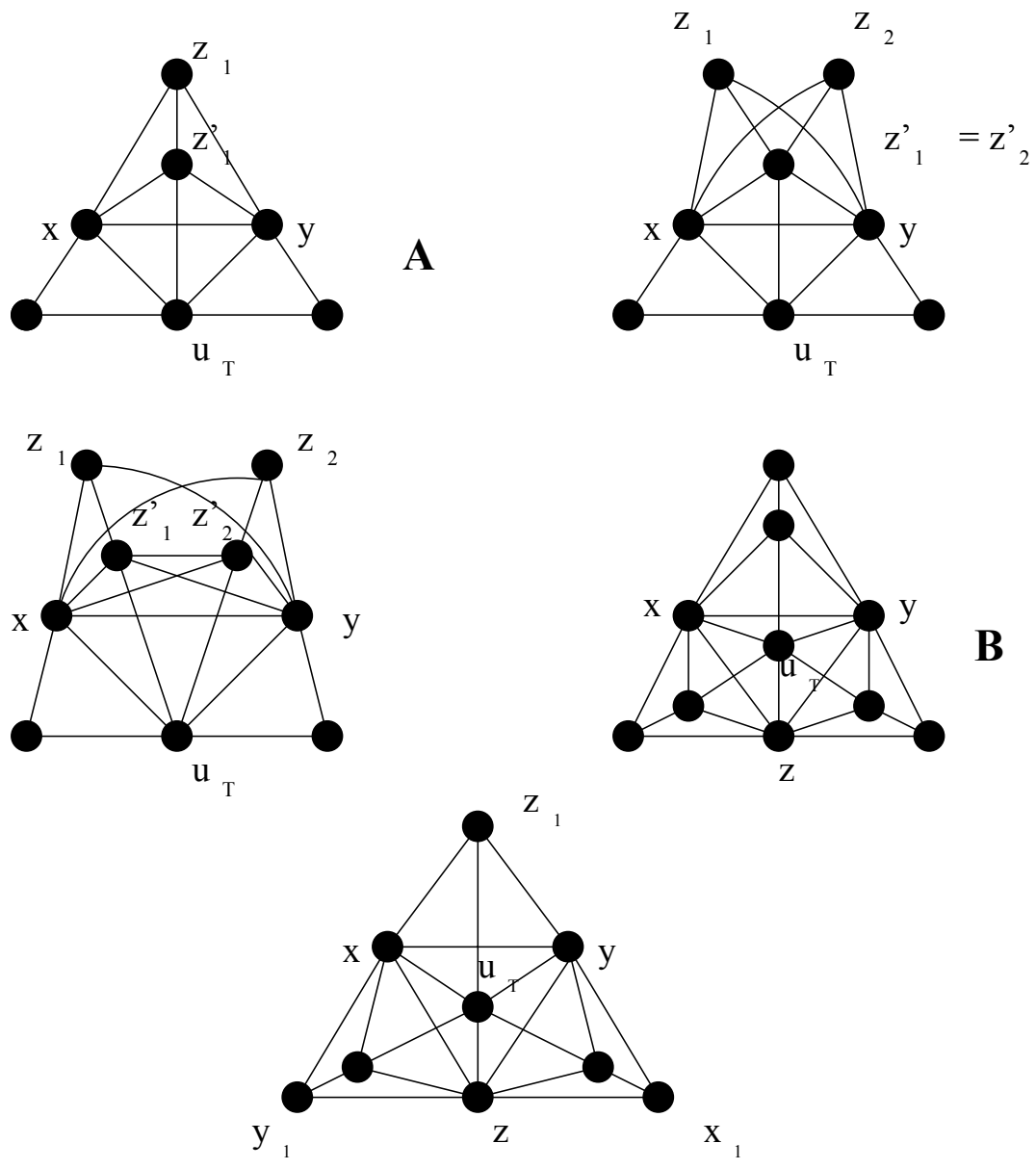

Por otro lado puede observarse que a partir del Teorema 6.1.2, es fácil ver que los grafos $\mathbf{A}$ y $\mathbf{B}$ tienen una única familia $\mathcal{R}-\mathcal{S}$.

\section{Aparece un Octaedro}

Un Octaedro r-dimensional, $\mathbf{O}_{r}$, es un grafo con $2 r$ vértices, cada uno de ellos con grado $2 r-2$. Estos grafos son de interés en el estudio de la convergencia del operador clique [18]. Veamos como el Octaedro 4-dimensional aparece a partir de cierta condición en una buena asignación de un grafo. 
Proposición 6.3.1. Sea $\mathbf{G}$ un grafo $\mathbf{K}_{5}$ - libre y $T=\{x, y, z\} \in T(\mathbf{G})$ tal que $\left|V_{x y z}\right| \geq 2$. Si $\mathbf{u}$ es una buena asignación de $\mathbf{G}$, entonces $u_{T} \in T$ o $\mathbf{O}_{4}$ es subgrafo de $\mathbf{G}$, tal como se muestra en la siguiente figura.

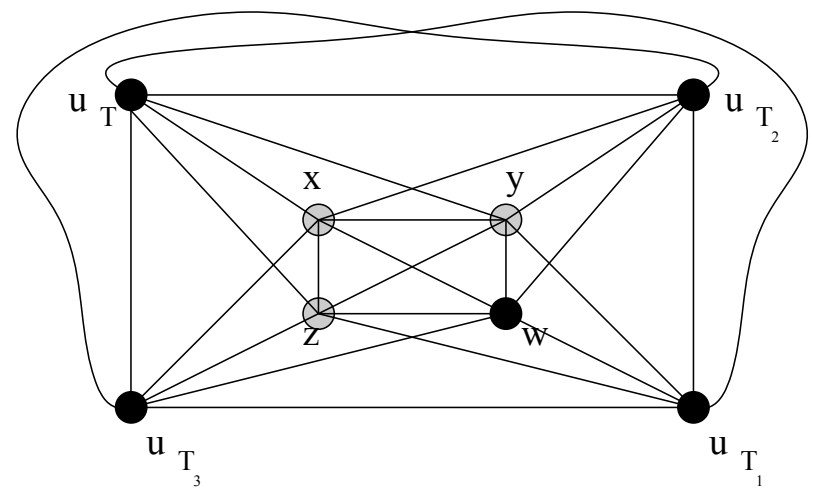

Demostración: Supongamos que $u_{T} \notin T$. Entonces $u_{T} \in V_{x y z}$.

Sea $w \neq u_{T}, w \in V_{x y z}$ y llamemos $T_{1}=\{y, z, w\}, T_{2}=\{x, y, w\}$ y $T_{3}=\{x, z, w\}$.

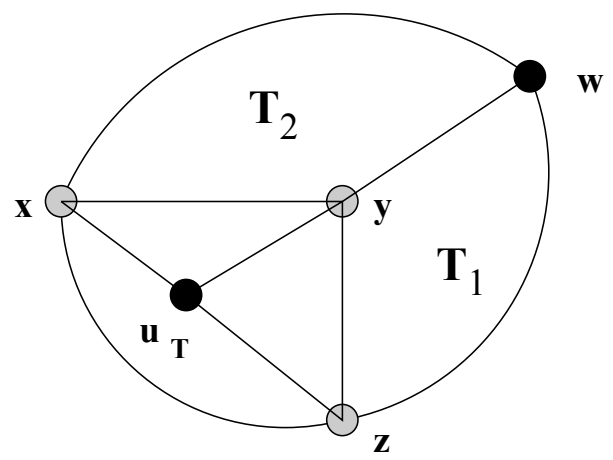

Como $w \not u_{T}$, se tiene que $u_{T_{1}} \neq u_{T}$ y además $u_{T_{1}} \notin T_{1}$. Por otra parte $u_{T_{1}} \neq x$, porque sino sería, por la condición 2 de la definición de asignación, $u_{T}=x$. Resulta, entonces, la situación descripta en la siguiente figura: 


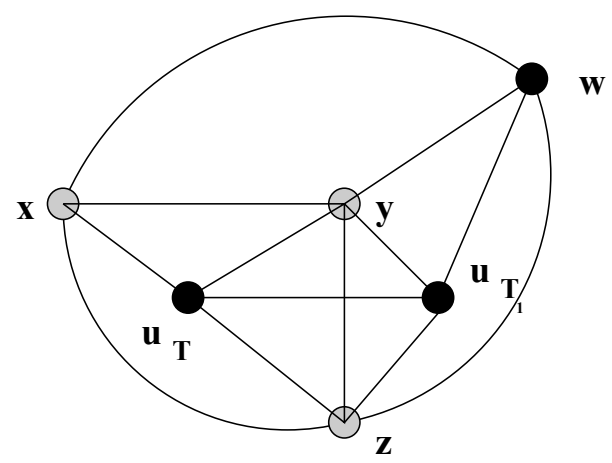

Análogamente, $u_{T_{2}} \neq u_{T}, u_{T_{2}} \notin T_{2}, \mathrm{y} u_{T_{2}} \neq z$. Además como $x \not u_{T_{1}}$ resulta que $u_{T_{1}} \neq u_{T_{2}}$.

La situación es la descripta en la siguiente figura:

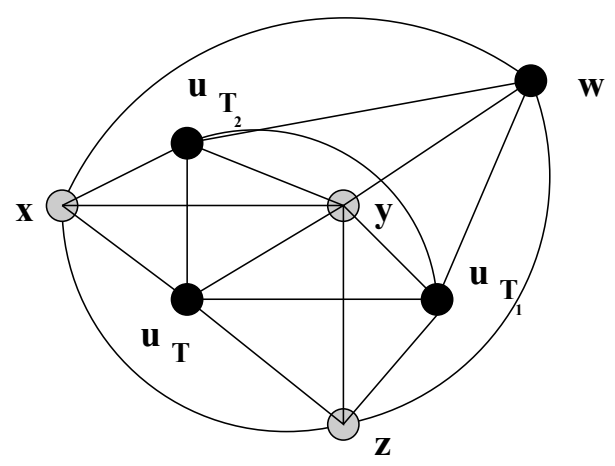

Análogamente resulta la existencia de $u_{T_{3}}$ y se completa la demostración. 


\section{Bibliografía}

[1] L. Alcón, M. Gutierrez, Clique Planar Graphs, Electronic Notes in Discrete Mathematic, Volumen 7.

[2] L. Alcón, M. Gutierrez, Cliques and Extended Triangles. A necessary condition to be Clique Planar Graph, aceptado en Discrete Applied Math.

[3] L. Alcón, M. Gutierrez, Clique Graphs of Planar Graphs, aceptado en Ars Combinatoria.

[4] L. Alcón, M. Gutierrez, A new characterization of Clique Graphs, aceptado en Matemática Contemporânea.

[5] R. Balakrishnan, P. Paulraja, Self-clique graphs and diameters of iterated clique graphs, Utilitas Mathematica, (1986),263-268.

[6] C.F. Borestein, J.L. Szwarcfiter, On clique convergent graphs, Graphs and Combinatorics, 11, (1995), 213-220.

[7] C.F. Borestein, J.L. Szwarcfiter, Iterated clique graphs with increasing diameters, Journal of Graph Theory, 28, (1998), 147-154.

[8] F.Escalante, Uber iterierte Clique Graphen, Abh. Math. Sem. Univ. Hamburg, 39, (1973), 59-68.

[9] M. Gutierrez, Intersection Graphs and the Clique Operator, Graphs and Combinatorics, 17, (2001), 237-244. 
[10] M. Gutierrez, J. Meidanis, Preimage, Image and Iterated Image of the Clique Operator, enviado a Discrete Mathematics,

[11] M. Gutierrez, J. Meidanis, On the Clique Operator, Lecture Notes in Computer Sciencie, 1380, (1998), 261-272. Proceedings of the 3rd. Latin American Conference on Theorical Informatics.

[12] M. Gutierrez, J. Meidanis, On Clique Graph Recognition, Ars Combinatoria, 63, (2002), 207-210.

[13] M. Gutierrez, J. Meidanis, Algebraic Theory for the Clique Operator, Journal of the Brazilian Computer Society, special issue dedicated to J.L. Szwarcfiter, Number 3, 7, 2002.

[14] R.C. Hamelink, A partial characterization of clique graphs, Journal of Combinatorial Theory, 5, (1968), 192-197.

[15] m.Ch. Lin, Grafos self-clique y otras clases de grafos clique, Tesis Doctoral,Universidad de Buenos Aires,(2001).

[16] V.Neumann-Lara, On clique-divergent graphs, en Problemes Combinatoires et Theorie des Graphes, Orsay, France, 1976. Colloques Internationaux C.N.R.S. 260.

[17] T. Nishizeki, N. Chiba, Planar Graphs: Theory and Algorithms, Annals of Discrete Mathematics, 32, (1988), North-Holland.

[18] M.A. Pizaña, Tesis Doctoral, Universidad Autónoma Metropolitana, México, (2002).

[19] E. Prisner, Convergence of iterated clique graphs, Discrete Mathematics, 103, (1992), 199-207.

[20] E. Prisner, J.L. Szwarcfiter, Recognizing clique graphs of directed and rooted path graphs, Discrete Applied Mathematics, 94, (1999), 321-328. 
[21] F. Protti, J.L. Szwarcfiter, On Clique Graphs with Linear Size, Relatorio Tecnico de la U.F.R.J., (NCE- 07/99).

[22] F. S. Roberts, J. H. Spencer, A characterizations of clique graphs, Journal of Combinatorial Theory B, 10, (1971), 102-108.

[23] J. Sedlác̆ek, Some properties of interchange graphs, en Theory of Graphs and its Applications, M. Fielder ed., Academic Press, New York, (1962), 145-150.

[24] M.M. Syslo, Some properties of interchange graphs, en Problemes Combinatoires et Theorie des Graphes, Orsay, France, 1976. Colloques Internationaux C.N.R.S. 260.

[25] J. L. Szwarcfiter, Recognizing Clique Helly Graphs, Ars Combinatoria 45, (1997), 29-32

[26] J. L. Szwarcfiter, A survey on Clique Graphs, in Recent Advances in Algorithms and Combinatorics,C. Linhares and B. Reed, eds.,Springer-Verlag, to appear.

[27] J. L. Szwarcfiter, C.F. Borestein, Clique graphs of chordal and path graphs, Order 13, (1996), 219-225. 